DANIELA BRASIL SILVA

Restauração cega de imagens: soluções baseadas em algoritmos adaptativos 
DANIELA BRASIL SILVA

Restauração cega de imagens: soluções baseadas em algoritmos adaptativos

Dissertação apresentada à Escola Politécnica da Universidade de São Paulo para obtenção do título de Mestre em Ciências. 
DANIELA BRASIL SILVA

\section{Restauração cega de imagens: soluções baseadas em algoritmos adaptativos}

Dissertação apresentada à Escola Politécnica da Universidade de São Paulo para obtenção do título de Mestre em Ciências.

Área de concentração:

Engenharia de Sistemas Eletrônicos

Orientador:

Prof. Dr. Magno Teófilo Madeira da Silva 
Este exemplar foi revisado e corrigido em relação à versão original, sob responsabilidade única do autor e com a anuência de seu orientador.

São Paulo, 03 de julho de 2018.

Daniela Brasil Silva

Prof. Dr. Magno Teófilo Madeira da Silva

Catalogação-na-publicação

Silva, Daniela Brasil

Restauração cega de imagens: soluções baseadas em algoritmos adaptativos/

D. B. Silva. -- versão corr. -- São Paulo, 2018. $127 \mathrm{p}$.

Dissertação (Mestrado) - Escola Politécnica da Universidade de São Paulo. Departamento de Engenharia de Sistemas Eletrônicos.

1. Imagens (Restauração) 2. Filtros elétricos adaptativos 3. Processamento de imagens 4. Imagem-Equalização I.Universidade de São Paulo. Escola Politécnica. Departamento de Engenharia de Sistemas Eletrônicos II.t 
Aos meus pais,

Wilson e Maria José 


\section{AGRADECIMENTOS}

Agradeço ao meu orientador, Prof. Dr. Magno Teófilo Madeira da Silva, sempre presente e atencioso, pela oportunidade e apoio imprescindíveis à realização deste trabalho. Obrigada pela atenção e paciência, e pela confiança depositada em mim desde o início do Mestrado.

Aos Profs. Drs. João Mendes Filho e Aline de Oliveira Neves Panazio, pela colaboração na banca examinadora.

Aos meus pais, Wilson e Maria José, pelo amor incondicional, por todo o cuidado, carinho, dedicação e por priorizarem a minha educação, que foi de fundamental importância para essa conquista.

Às minhas irmãs Adriana, Patrícia, Maria e Renata, pelo incentivo, amizade, força e risos constantes. Ao meu companheiro, Thiago, por toda a força, compreensão e amor.

Aos amigos da Elétrica, pelos momentos alegres que ajudaram a tornar essa jornada mais leve. Em especial ao amigo Dante, pelas conversas agradáveis, pelos conselhos e por sua disponibilidade em ajudar.

À Coordenação de Aperfeiçoamento de Pessoal de Nível Superior (CAPES), pelo financiamento deste trabalho. 


\section{RESUMO}

O objetivo da desconvolução cega de imagens é restaurar uma imagem degradada sem usar informação da imagem real ou da função de degradação. O mapeamento dos níveis de cinza de uma imagem em um sinal de comunicação possibilita o uso de técnicas de equalização cega de canais para a restauração de imagens. Neste trabalho, propõe-se o uso de um esquema para desconvolução cega de imagens baseado na combinação convexa de um equalizador cego com um equalizador no modo de decisão direta. A combinação também é adaptada de forma cega, o que possibilita o chaveamento automático entre os filtros componentes. Dessa forma, o esquema proposto é capaz de atingir o desempenho de um algoritmo de filtragem adaptativa supervisionada sem o conhecimento prévio da imagem original. O desempenho da combinação é ilustrado por meio de simulações, que comprovam a eficiência desse esquema quando comparado a outras soluções da literatura.

Palavras-chave: Restauração cega de imagens. Combinação de algoritmos adaptativos. Algoritmo do módulo constante. Modo de decisão direta. Filtragem adaptativa. 


\begin{abstract}
The goal of blind image deconvolution is to restore a degraded image without using information from the actual image or from the point spread function. The mapping of the gray levels of an image into a communication signal enables the use of blind equalization techniques for image restoration. In this work, we use a blind image deconvolution scheme based on the convex combination of a blind equalizer with an equalizer in the decision-directed mode. The combination is also blindly adapted, which enables automatic switching between the component filters. Thus, the proposed scheme is able to achieve the performance of a supervised adaptive filtering algorithm without prior knowledge of the original image. The performance of the combination is illustrated by simulations, which show the efficiency of this scheme when compared to other solutions in the literature.
\end{abstract}

Keywords: Blind image restoration. Combination of adaptive algorithms. Constant modulus algorithm. Decision-directed mode. Adaptive filtering. 


\section{LISTA DE ABREVIAÇÕES}

CMA

DD

DFE

FIR

iid

$|S|$

KAF

KLMS

LMS

LTE

MAP

$M L$

MSE

\%MSE

MSSIM

NLMS

PSF

QAM

QKLMS

RMA

SER

SNR

TDLMS constant modulus algorithm (algoritmo do módulo constante)

decision directed (decisão direta)

decision feedback equalizer (equalizador de decisão realimentada)

finite impulse response (resposta ao pulso unitário finita)

independente e identicamente distribuído

intersymbol interference (interferência intersimbólica)

kernel adaptive filters (filtro adaptativo baseado em núcleo)

kernel least mean squares

least mean squares

linear transversal equalizer (equalizador linear transversal)

maximum a posteriori probability (máxima probabilidade a posteriori)

maximum likelihood (máxima verossimilhança)

mean square error (erro quadrático médio)

erro quadrático médio percentual

mean structural similarity (similaridade estrutural média)

normalized least mean squares

point spread function (função de espalhamento de ponto)

quadrature amplitude modulation (modulação de amplitude em quadratura)

quantized kernel least mean squares

regional multimodulus algorithm (algoritmo multimódulo regional)

symbol error rate (taxa de erro de símbolo)

signal-noise ratio (razão sinal-ruído)

two-dimensional LMS 


\section{LISTA DE SÍMBOLOS}

\section{Símbolos gerais}
$(\cdot)^{T} \quad$ transposição de vetores ou matrizes
$\mathrm{E}\{\cdot\} \quad$ operador esperança matemática
$\|\cdot\| \quad$ norma euclidiana de um vetor
$\mathbb{R} \quad$ conjunto dos números reais

\section{Equalização adaptativa}

$n$

amostra de tempo

$a$

símbolo transmitido

$x$

sinal degradado por um canal

$H(z) \quad$ função de transferência do canal

$\xi \quad$ ruído branco gaussiano

$u \quad$ sinal de entrada do equalizador

$\Delta \quad$ atraso em número de amostra

$\mathbf{u} \quad$ vetor regressor de entrada do equalizador

w vetor de coeficientes do equalizador

M número de coeficientes do equalizador

y sinal de saída do equalizador

decisor [·] função decisor

$\hat{a} \quad$ sinal de saída decidido

e erro de estimação de algoritmos supervisionados

$e_{d} \quad$ erro de decisão de algoritmos supervisionados

erro de decisão global da combinação 


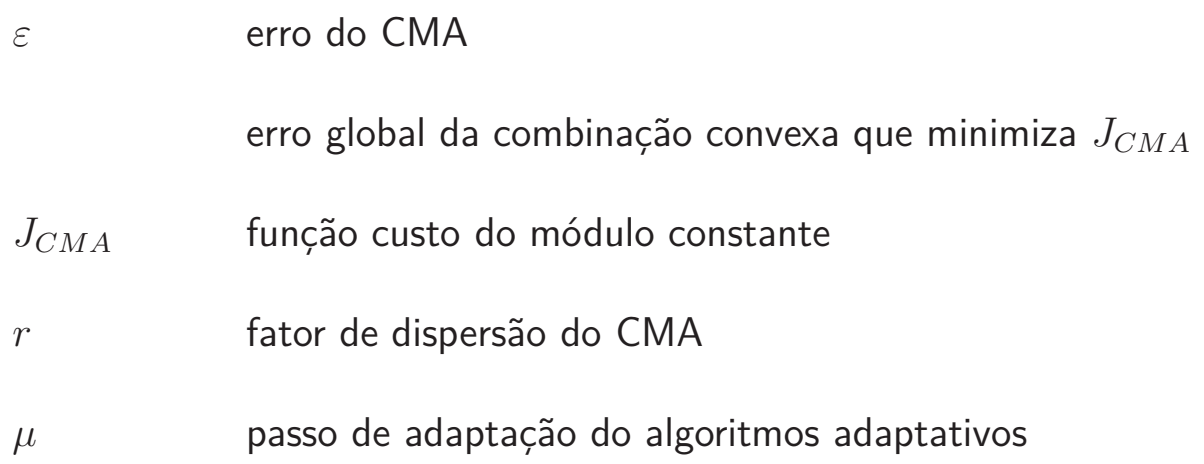

\section{Equalizador de decisão realimentada}

$\begin{array}{ll}\mathbf{w}_{f} & \text { vetor de coeficientes do filtro direto } \\ \mathbf{w}_{b} & \text { vetor de coeficientes do filtro de realimentação } \\ \mathbf{w}_{f b} & \text { vetor de coeficientes dos filtros concatenados } \\ M_{f} & \text { número de coeficientes do filtro direto } \\ M_{b} & \text { número de coeficientes do filtro de realimentação } \\ M_{f b} & \text { número de coeficientes dos filtros concatenados } \\ y_{f} & \text { sinal de saída do filtro direto } \\ y_{b} & \text { vinal de saída do filtro de realimentação } \\ \hat{\mathbf{a}}_{\Delta} & \text { vetor regres com as decisões } \hat{a}\end{array}$

\section{Filtro adaptativo baseado em kernel}

$\mathbb{F}$ espaço das características

$\kappa(\cdot, \cdot) \quad$ kernel

$\Phi(\mathrm{x}) \quad$ função de mapeamento de $\mathrm{x}$ em $\mathbb{F}$

$\Omega \quad$ vetor de coeficientes do filtro

b vetor de coeficientes que ponderam $\kappa(\cdot, \cdot)$

C dicionário do KAF

$\rho \quad$ limiar do dicionário no QKLMS 
$N_{c} \quad$ cardinalidade do dicionário $\mathbf{C}$

\section{Combinação convexa}

$\begin{array}{ll}\lambda & \text { parâmetro de mistura da combinação } \\ y_{1} \text { e } y_{2} & \text { saídas dos filtros componentes da combinação } \\ \mathbf{w}_{1} \text { e } \mathbf{w}_{2} & \text { vetores de coeficientes dos componentes da combinação } \\ \varepsilon_{1} & \text { erro do CMA do equalizador cego da combinação } \\ e_{d 2} & \text { erro de decisão do equalizador DD da combinação } \\ \mu_{1} \text { e } \mu_{2} & \text { passos de adaptação dos componentes da combinação } \\ \alpha & \text { variável auxiliar da combinação convexa } \\ \alpha^{+} & \text {valor máximo permitido para }|\alpha| \\ \varphi^{\prime}[\cdot] & \text { função de ativação da combinação convexa } \\ \varphi^{\prime}[\cdot] & \text { derivada de } \varphi[\cdot] \\ \operatorname{sgm}[\cdot] & \text { função sigmoidal } \\ \mu_{\alpha} & \text { passo de adaptação de } \alpha \\ p & \text { função sinal } \\ \operatorname{sign}[\cdot] & \text { estimativa da potência de }\left[y_{1}-y_{2}\right]\end{array}$

\section{Restauração de imagens}

$\mathbf{F}\left(k_{1}, k_{2}\right) \quad$ imagem original

$\widehat{\mathbf{F}}\left(k_{1}, k_{2}\right) \quad$ estimativa da imagem original

$\mathbf{G}\left(k_{1}, k_{2}\right) \quad$ imagem degradada

$\mathbf{H}\left(k_{1}, k_{2}\right) \quad$ função de espalhamento de ponto

$\Upsilon\left(k_{1}, k_{2}\right) \quad$ ruído aditivo de uma imagem 
$\mathbf{U}_{n} \quad$ janela de varredura do filtro adaptativo

$\operatorname{vec}[\cdot] \quad$ função que vetoriza a janela $\mathbf{U}_{n}$

c ciclos de varredura sobre a imagem

$\bar{\lambda} \quad$ valor médio do parâmetro de mistura $\lambda$ ao longo dos ciclos de varredura 


\section{SUMÁRIO}

1 Introdução e Formulação do Problema 1

1.1 Objetivos e justificativa . . . . . . . . . . . . . . . . . 5

1.2 Organização do trabalho . . . . . . . . . . . . . . . . 6

1.2 .1 Artigo publicado . . . . . . . . . . . . . . . . . . . . . 6

2 Algoritmos adaptativos para equalização de canais de comunicação

2.1 Equalização de canais de comunicação . . . . . . . . . . . . . . . . . . . 7

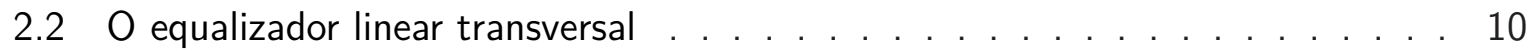

2.3 O equalizador de decisão realimentada f . . . . . . . . . . . . . . . . . . . . . . . . . . 14

$2.4 \mathrm{O}$ equalizador com filtro adaptativo baseado em kernel . . . . . . . . . . . . 16

2.4 .1 O algoritmo KLMS . . . . . . . . . . . . . . . . . . . 17

2.4 .2 O algoritmo QKLMS . . . . . . . . . . . . . . . . 20

2.5 Conclusão . . . . . . . . . . . . . . . . . . . . . . . . . . . . . . . . . 21

3 Combinação convexa de filtros adaptativos para equalização cega 23

3.1 Combinação convexa entre equalizadores cegos e equalizadores no modo DD . 23

3.1.1 Combinação com minimização da função custo do módulo constante . 26

3.1.2 Combinação com minimização do erro quadrático médio de decisão . . 26

3.1.3 Resultados de simulações . . . . . . . . . . . . . . . . . . . . . . 29

3.1.3.1 Canais não baseados em PSF . . . . . . . . . . . . . . . . 29

3.1.3.2 Canais baseados em PSF . . . . . . . . . . . . . . 33

3.2 Combinação convexa baseada em soluções não lineares para equalização . . . . 43

3.2.1 Combinação convexa entre o CMA e o DFE-NLMS-DD . . . . . . . . 43 
3.2.2 Combinação convexa entre o CMA e o QKLMS-DD . . . . . . . . . . 46

3.2.3 Resultados de simulações . . . . . . . . . . . . . . . . . . . . . . 49

3.3 Conclusão . . . . . . . . . . . . . . . . . . . . . . . . . . . . . . . . . . . 72

4 Restauração de imagens $\quad 74$

4.1 Formulação do problema . . . . . . . . . . . . . . . . . . . . . . . . . . . . 74

4.2 Procedimentos para o uso de algoritmos adaptativos na restauração de imagens 76

4.2 .1 Definição de janelas . . . . . . . . . . . . . . . . . 76

4.2 .2 Interfaces de borda . . . . . . . . . . . . . . . . . . 76

4.2 .3 Percurso de varredura . . . . . . . . . . . . . . . . . . . . . 77

4.2.4 Imagem como sinal unidimensional . . . . . . . . . . . . . . . . . 78

4.3 Restauração cega de imagens com a combinação de equalizadores adaptativos 79

4.4 Resultados de simulações . . . . . . . . . . . . . . . . . . . . . 81

4.4 .1 Parâmetros de avaliação . . . . . . . . . . . . . . . . . . . . . . 81

4.4 .2 PSFs utilizadas . . . . . . . . . . . . . . . . . . . . . . . . . . 82

4.4.3 Resultados da combinação do CMA com o NLMS-DD . . . . . . . . . 83

4.4.4 Resultados da combinação do CMA com equalizadores não lineares . . 96

4.5 Conclusão . . . . . . . . . . . . . . . . . . . . . . . . . . . . . . . . . . . . . . 99

5 Conclusões $\quad 101$

$\begin{array}{ll}\text { Referências } & 103\end{array}$

$\begin{array}{ll}\text { Apêndice A - O algoritmo RMA } & 110\end{array}$ 


\section{INTRODUÇÃO E FORMULAÇÃO DO PROBLEMA}

A restauração de imagens tem como objetivo recuperar a imagem original a partir da sua versão degradada. A restauração é um problema tipicamente mal posto, o que significa que sua solução não satisfaz ao menos um dos seguintes critérios: existência, singularidade ou estabilidade (BANHAM; KATSAGGELOS, 1997; BERTERO; BOCCACCI, 1998). Ela é amplamente utilizada em diversas areas técnicas que envolvem imagens, como por exemplo, astronomia (BERTERO; BOCCACCI, 2000), sensoriamento remoto (JENSEN; LULLA, 1987), microscopia (WALLACE et al., 2018), imagens médicas (JEFFERIES et al., 2002; BRONSTEIN et al., 2004; ADAM; MICHAILOVICH, 2002), superresolução (HUANGPENG et al., 2015; FU et al., 2016), entre outras.

Uma imagem digital é a representação discreta de uma imagem real, segmentada em pixels (picture elements). Cada pixel possui uma amplitude quantizada referente a um nível de cinza, que representa a cor da imagem real nesse ponto. $O$ conjunto desses pixels formam a imagem digital. Geralmente, as características de uma imagem natural são mais bem representadas utilizando 256 níveis de cinza, que correspondem a uma imagem digital de 8-bits/pixel (GONZALEZ; WOODS, 2008).

Uma imagem pode sofrer diferentes tipos de degradação provenientes do processo de aquisição (GONZALEZ; WOODS, 2008). Essas degradações aparecem nas mais diversas aplicações, podendo ser resultado do processo de quantização, compressão, saturação, desfocagem ou movimento da câmera durante a exposição, imperfeições óticas das lentes de instrumentos, entre outras fontes (CAMPISI; EGIAZARIAN, 2007). Geralmente, essas degradações são processos não lineares e variantes no espaço. No entanto, muitos métodos de restauração utilizam o modelo que trata a degradação como linear e invariante no espaço (GUNTURK; LI, 2017; CAMPISI; EGIAZARIAN, 2007), conforme é esquematizado na Figura 1. Nesse esquema, a imagem degradada é o sinal bidimensional representado pela matriz $\mathbf{G}$, cujo elemento $\left(k_{1}, k_{2}\right)$ representa um pixel da imagem, ou seja, $\mathbf{G}\left(k_{1}, k_{2}\right)$. No modelo, $\mathbf{G}\left(k_{1}, k_{2}\right)$ é definido por

$$
\mathbf{G}\left(k_{1}, k_{2}\right)=\mathbf{F}\left(k_{1}, k_{2}\right) * \mathbf{H}\left(k_{1}, k_{2}\right)+\Upsilon\left(k_{1}, k_{2}\right),
$$

isso é, resultado da convolução, representada por $*$, entre a imagem original $\mathbf{F}\left(k_{1}, k_{2}\right)$ e a 
função de espalhamento de ponto (PSF - point spread function) $\mathbf{H}\left(k_{1}, k_{2}\right)$, somada ao ruído $\Upsilon\left(k_{1}, k_{2}\right)$, geralmente assumido branco, gaussiano e de média zero.

Figura 1 - Representação do modelo de degradação linear e invariante no espaço para imagens.

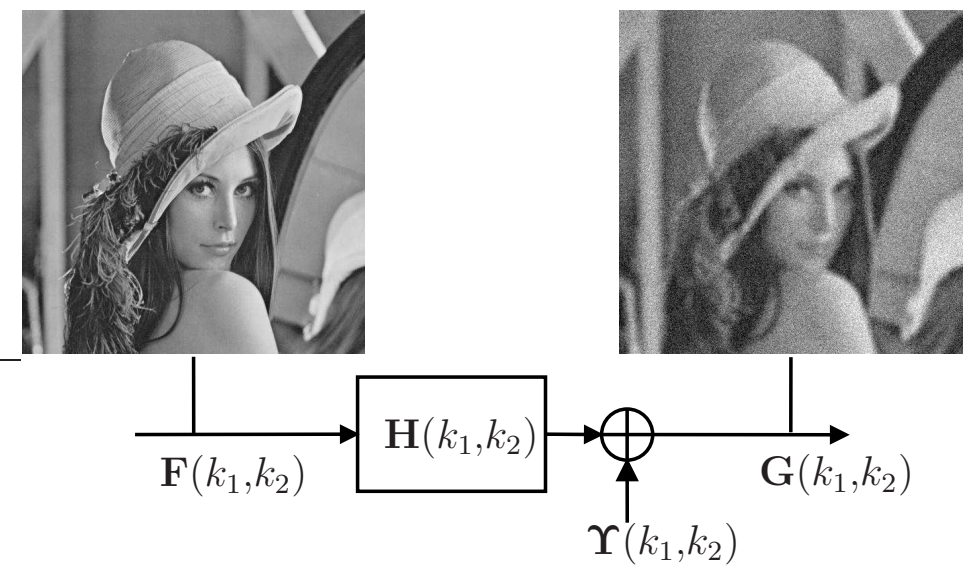

Fonte: Autora.

Na ausência de ruído, a restauração de imagens é chamada de desconvolução de imagens, uma vez que seu objetivo é reverter o efeito da convolução entre a imagem original e a PSF. A minimização do efeito do ruído é conhecida na literatura como denoising (GUNTURK; LI, 2017).

Os métodos de restauração de imagens podem ser divididos em dois grupos principais: os métodos não cegos, que assumem que a PSF é conhecida, e os métodos cegos, situação mais realista em que tanto a imagem quanto a PSF são desconhecidas total ou parcialmente (KUNDUR; HATZINAKOS, 1996a; KUNDUR; HATZINAKOS, 1996b). Na restauração não cega, deseja-se obter uma estimativa $\widehat{\mathbf{F}}\left(k_{1}, k_{2}\right)$ da imagem original conhecendo-se $\mathbf{G}\left(k_{1}, k_{2}\right)$ e $\mathbf{H}\left(k_{1}, k_{2}\right)$. À primeira vista, parece um problema simples. No entanto, os métodos existentes são muito sensíveis às variações entre a PSF usada e a real, o que significa que um conhecimento pobre a respeito da PSF leva a resultados ruins. Além disso, como a PSF é geralmente mal condicionada, a presença do ruído $\Upsilon\left(k_{1}, k_{2}\right)$, mesmo que fraca, pode afetar severamente os resultados da restauração (KUNDUR; HATZINAKOS, 1996a). Como exemplos dessas técnicas, têm-se o filtro de Wiener e a filtragem de mínimos quadrados com restrição (GONZALEZ; WOODS, 2008), que são técnicas tradicionalmente conhecidas pela simplicidade de implementação e pelo baixo custo computacional requerido. Além desses algoritmos, destaca-se o algoritmo de Richardson-Lucy, proposto independentemente por (RICHARDSON, 1972) e (LUCY, 1974), que é uma solução iterativa não linear que requer mais recursos computacionais e produz resultados superiores ao dos métodos tradicionais. 
Na restauração cega, ou desconvolução cega, busca-se estimar $\widehat{\mathbf{F}}\left(k_{1}, k_{2}\right)$ conhecendo-se apenas a imagem degradada $\mathbf{G}\left(k_{1}, k_{2}\right)$. Este é um problema mal posto, uma vez que para uma dada imagem $\mathbf{G}\left(k_{1}, k_{2}\right)$ podem existir infinitas soluções para $\mathbf{F}\left(k_{1}, k_{2}\right)$ e $\mathbf{H}\left(k_{1}, k_{2}\right)$, ou até mesmo nenhuma solução, ocasionando instabilidade numérica aos métodos de resolução. Existem diferentes abordagens para lidar com o problema da desconvolução cega na literatura, sendo que a maioria delas pode ser vista como casos especiais da aplicação do teorema de Bayes (CAMPISI; EGIAZARIAN, 2007). As principais diferenças entre elas são as escolhas da função de otimização e da distribuição de probabilidade a priori usada para modelar a imagem original e o processo de degradação, em que são utilizados métodos estatísticos de estimação, como a máxima verossimilhança (maximum likelihood - ML) e a máxima probabilidade a posteriori (maximum a posteriori probability - MAP) (CAMPISI; EGIAZARIAN, 2007).

Os métodos de desconvolução cega podem ser classificados em dois tipos: aqueles em que a PSF é identificada a priori e aqueles em que a PSF é identificada juntamente com a imagem. No primeiro tipo, a PSF é identificada separadamente da imagem, sendo combinada com algum algoritmo de restauração não cega para obter a estimativa final da imagem original. Esses métodos buscam estimar a PSF mais provável a partir da imagem degradada e funcionam melhor em situações nas quais emprega-se um modelo paramétrico para a PSF. Essa abordagem é explorada em trabalhos como (FERGUS et al., 2006; XU; JIA, 2010; GOLDSTEIN; FATTAL, 2012; OLIVEIRA; FIGUEIREDO; BIOUCAS-DIAS, 2014). No segundo tipo de desconvolução cega, a imagem e a PSF são estimadas simultaneamente. Apesar de ser computacionalmente mais complexo, é o método mais adequado considerando os diferentes tipos de degradação e por isso, sua abordagem é mais recorrente na literatura (CHAN; WONG, 1998; LIKAS; GALATSANOS, 2004; BRONSTEIN et al., 2005; MOLINA; MATEOS; KATSAGGELOS, 2006; MONEY; KANG, 2008; ALMEIDA; ALMEIDA, 2008; AMIZIC et al., 2010; ALMEIDA; ALMEIDA, 2010).

Nesta dissertação, utilizam-se filtros adaptativos para estimar a PSF e a imagem simultaneamente. Dentre outras aplicações, os filtros adaptativos são utilizados na equalização de canais de comunicação com o objetivo de reverter a degradação causada pelo canal, recuperando o sinal transmitido (HAYKIN, 2002). Quando empregado na restauração de imagens, os filtros adaptativos são estendidos para o caso bidimensional e sua função é inverter a PSF, desconvoluindo a imagem degradada. Essa abordagem é discutida em (HADHOUD; THOMAS, 1998), onde o algoritmo LMS (least mean squares) é estendido para duas dimensões resul- 
tando no algoritmo TDLMS (two-dimensional LMS) para aplicação em imagens. No entanto, esse algoritmo requer a informação do sinal de imagem original como sinal desejado, não disponível em aplicações práticas. Assim, uma solução mais interessante é a proposta em (VURAL; SETHARES, 2006), em que o algoritmo do módulo constante (constant modulus algorithm - CMA), amplamente usado em equalização cega de canais de comunicação, é estendido para a desconvolução cega de imagens.

Na equalização de canais de comunicação, o CMA é o mais popular para adaptação de equalizadores de resposta ao pulso finita e é amplamente utilizado mesmo para constelações de módulo não constante, como é o caso de N-PAM (Pulse Amplitude Modulation) com $N>2$ (JOHNSON-JR. et al., 1998). Embora o CMA possua um custo computacional baixo, seu principal problema é a convergência para mínimos locais indesejáveis. Nesse caso, ele pode atingir um nível de erro quadrático médio (mean square error - MSE) alto em regime. Além disso, o CMA pode atingir um MSE igual a zero apenas para sinais de módulo constante em um ambiente estacionário e sem ruído e considerando sobreamostragem (ver, e.g., (SILVA; NASCIMENTO, 2008)). Diante disso, é comum chavear o modo de treinamento cego com o CMA para o modo de decisão direta (decision directed - DD). No modo DD, a decisão tomada sobre a saída do equalizador faz o papel de sinal desejado, que por sua vez, é utilizado em um algoritmo de filtragem adaptativa supervisionada, como o algoritmo NLMS (normalized least mean squares), por exemplo (SAYED, 2003). O bom desempenho global do equalizador depende da seleção do limiar de MSE adequado para o chaveamento (JOHNSON-JR. et al., 2000). No entanto, essa não é uma tarefa simples, já que esse limiar depende da constelação do sinal transmitido, do canal de comunicação, da razão sinal-ruído (noise-signal ratio - SNR), entre outros fatores (JOHNSON-JR. et al., 2000). Para evitar a necessidade de se selecionar um limiar de MSE para o chaveamento abrupto (hard switching) entre os modos cego e de decisão direta, vários esquemas de chaveamento suave foram propostos na literatura (ver, e.g., (CASTRO; CASTRO; ARANTES, 2001; CHEN, 2003)). Além disso, algoritmos de equalização cega com bom desempenho no transitório e em regime têm sido propostos a fim de evitar o mecanismo de chaveamento para o modo DD (ver, e.g., (MENDES-FILHO; MIRANDA; SILVA, 2012) e suas referências). Entretanto, esses esquemas são tipicamente difíceis de ajustar e o desempenho alcançado ainda é dependente do ambiente particular em que são aplicados. Nesse contexto, foi proposto em (SILVA; ARENAS-GARCÍA, 2013) um esquema que combina de forma adaptativa um equalizador cego com um equaliza- 
dor no modo de decisão direta funcionando em paralelo. A combinação é adaptada de forma cega e, consequentemente, o esquema proposto possibilita um chaveamento automático entre os filtros componentes, evitando a seleção a priori de um nível de MSE para a transição. As simulações de (SILVA; ARENAS-GARCÍA, 2013) indicam que o desempenho desse esquema supera os de outros equalizadores com chaveamento suave existentes na literatura em termos de velocidade de convergência e MSE em regime.

Embora a abordagem de (VURAL; SETHARES, 2006) seja inovadora, alguns avanços recentes em algoritmos cegos para equalização de canais de comunicação podem ser estendidos para restauração cega de imagens para se obter uma redução da deterioração de forma mais efetiva. Nesse sentido, os trabalhos (ABREU; SILVA, 2011; MENDES-FILHO; MIRANDA; SILVA, 2014) estenderam o algoritmo de multimódulo regional (regional multimodulus algorithm - RMA, ver Apêndice A para mais detalhes) de (MENDES-FILHO; MIRANDA; SILVA, 2012) para a desconvolução cega de imagens. Apesar do desempenho do RMA para restauração de imagens ser melhor que o do CMA de (VURAL; SETHARES, 2006), notou-se, a partir das simulações de (MENDES-FILHO; MIRANDA; SILVA, 2014), a necessidade de um critério de parada do algoritmo para evitar degradação do desempenho, similar ao que ocorre com as técnicas de (ALMEIDA; ALMEIDA, 2010; ALMEIDA; FIGUEIREDO, 2013). Como o esquema de (SILVA; ARENAS-GARCÍA, 2013) apresenta um desempenho melhor que o RMA em equalização de canais de comunicação, espera-se que o mesmo aconteça na restauração de imagens, algo que ainda não foi explorado na literatura. Assim, propõe-se a extensão do esquema de (SILVA; ARENAS-GARCÍA, 2013) para desconvolução cega de imagens.

\subsection{Objetivos e justificativa}

Os objetivos deste trabalho são elencados a seguir:

- utilizar o esquema baseado na combinação convexa entre um equalizador cego e um equalizador no modo de decisão direta para aprimorar o desempenho da restauração cega de imagens com filtros adaptativos;

- investigar novas soluções para o equalizador no modo de decisão direta, de maneira que a combinação possa apresentar um desempenho melhor em canais mais difíceis de 
equalizar (como por exemplo, os canais não lineares) e dessa forma estender essa solução para outros tipos de degradação.

\subsection{Organização do trabalho}

Como a abordagem utilizada para a restauração de imagens provém de técnicas de equalização de canais, decidiu-se dedicar os Capítulos 2 e 3 para revisitar essas técnicas e ao mesmo tempo, no Capítulo 3, propor novos esquemas, que são estendidos para o uso em imagens no Capítulo 4.

Assim, o Capítulo 2 aborda a equalização de canais de comunicação com algoritmos adaptativos, onde inicialmente os conceitos gerais sobre equalização de canais são apresentados, definindo-se o esquema de um sistema de comunicação e o modelo do canal. Adicionalmente, são discutidas algumas soluções ótimas baseadas em critérios recorrentes na literatura. $E$ então, a equalização com filtros adaptativos é apresentada, definindo-se os modos de treinamento supervisionado, cego e o modo de decisão direta. Além disso, discutem-se soluções adaptativas não lineares que podem ser mais adequadas na equalização de canais com nulos espectrais e/ou não linearidade.

O Capítulo 3 apresenta o esquema da combinação convexa entre um equalizador cego e um equalizador no modo de decisão direta. Além disso, propõe-se o uso das soluções não lineares discutidas no Capítulo 2 para o equalizador no modo de decisão direta.

No Capítulo 4, os esquemas de combinação discutidos no Capítulo 3 são estendidos para a restauração cega de imagens. Assim, são mostrados os resultados de simulações, comparandoos com outras soluções existentes.

Por fim, no Capítulo 5, são apresentadas as conclusões do trabalho realizado e as sugestões para trabalhos futuros.

\subsubsection{Artigo publicado}

Daniela B. Silva, Magno T. M. Silva: "Um esquema para restauração cega de imagens baseado em combinação de algoritmos adaptativos.", Anais do Simpósio Brasileiro de Telecomunicações e Processamento de Sinais, p. 1-5, 2017 (BRASIL-SILVA; SILVA, 2017). 


\section{ALGORITMOS ADAPTATIVOS PARA EQUALIZAÇÃO DE CANAIS DE COMUNICAÇÃO}

Este capítulo trata da equalização de canais de comunicação com algoritmos adaptativos. Na Seção 2.1, são apresentados o conceito geral de equalização, o modelo de canal, critérios de solução ótima e as soluções utilizadas neste trabalho. Na Seção 2.2, o modelo do equalizador linear transversal é discutido, assim como os tipos de equalização (modo supervisionado, de decisão direta e cego) e alguns algoritmos adaptativos. Na Seção 2.3, é mostrado o esquema do equalizador de decisão realimentada. Na Seção 2.4, discute-se a solução de filtragem adaptativa baseada em kernel para a equalização de canais não lineares. Por fim, na Seção 2.5, são feitas as considerações finais sobre o capítulo.

\subsection{Equalização de canais de comunicação}

Nos sistemas de comunicação digital, o sinal transmitido sofre degradações provenientes do meio de transmissão e portanto, é necessário o uso de um equalizador, cuja função é mitigar as degradações, para recuperar o sinal original no receptor (DING; LI, 2001; HAYKIN, 2002). A Figura 2 mostra o esquema da equalização, em que um sinal transmitido $a(n)$ é degradado por um canal de comunicação, resultando no sinal $u(n)$, entrada do equalizador. O equalizador gera a saída $y(n)$, que passa por um decisor, resultando na estimativa do sinal transmitido $\hat{a}(n-\Delta)$, que apresenta um atraso de $\Delta$ amostras.

Figura 2 - Esquema de um sistema de comunicação com um equalizador.

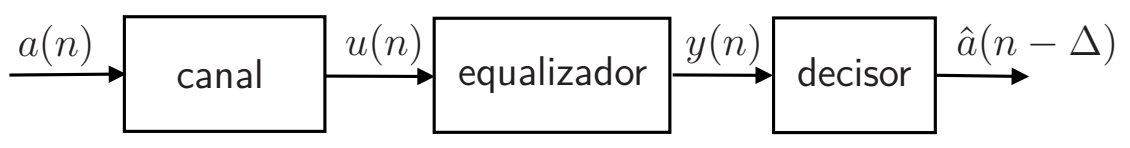

Fonte: Autora.

O modelo geral para o canal de comunicação é mostrado na Figura 3, em que há uma parte linear representada por um filtro de resposta ao pulso unitário finita (finite impulse response - FIR) com função de transferência $H(z)$, uma não linearidade representada pelo bloco "N.L." e um ruído aditivo $\xi(n)$. Esse modelo de canal não se refere apenas ao meio físico de transmissão mas também ao sistema de transmissão/modulação e o sistema recep- 
ção/demodulação. Em geral, os canais de comunicação são dispersivos devido aos diferentes atrasos na propagação do sinal, resultando na presença de interferência intersimbólica (intersymbol interference - ISI), representada pelo filtro $H(z)$ (PROAKIS; SALEHI, 2007). A não linearidade, presente em alguns casos, é geralmente causada pelo emprego de dispositivos não lineares, tais como amplificadores de potência e equipamentos ópticos, que podem deteriorar significativamente o sinal recebido, o que acontece por exemplo em canais de satélite (CRIPPS, 2006; JOHANNISSON; KARLSSON, 2013; POGGIOLINI et al., 2014).

Figura 3 - Modelo do canal de comunicação.

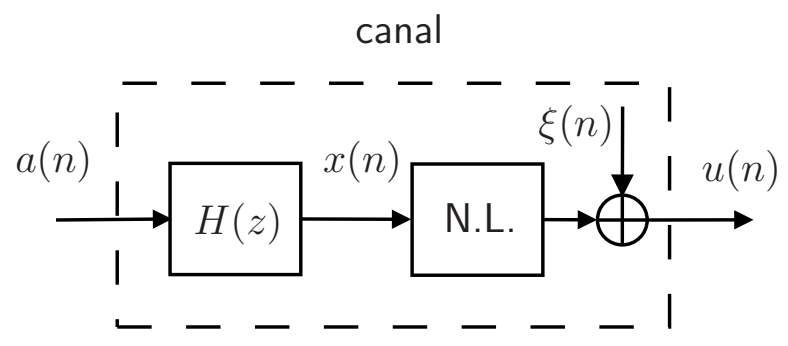

Fonte: Autora.

Considerando a equalização de um canal fixo, pode-se obter equalizadores ótimos segundo os diferentes critérios a seguir (FIGUEIRAS-VIDAL, 2012):

i. Critérios que minimizam momentos de $2^{\mathrm{a}}$ ordem, como a minimização do erro quadrático médio ou critério dos mínimos quadrados;

ii. Critério de Bayes (para maximização da probabilidade a posteriori do símbolo transmitido dado o sinal recebido);

iii. Critérios originários da teoria da informação, como o da máxima verossimilhança ou da máxima entropia.

O primeiro critério leva à solução de Wiener (HAYKIN, 2002), que depende da matriz de autocorrelação do sinal $u(n)$ e do vetor de correlação cruzada entre a sequência de treinamento $a(n-\Delta)$ e o sinal $u(n)$. A solução de Wiener depende do atraso $\Delta$ e muda se as estatísticas de $u(n)$ variarem, o que pode ocorrer em canais variantes no tempo (HAYKIN, 2002). Diante disso, é comum utilizar como equalizador um filtro adaptativo. Considerando um filtro adaptativo FIR, diz-se que o equalizador é do tipo linear transversal (linear transversal equalizer - LTE). É possível mostrar que em um ambiente estacionário um filtro adaptativo 
que minimiza o erro quadrático médio, por exemplo, é capaz de atingir a solução de Wiener na média. Cabe observar que em (HAYKIN, 2002), esta solução é tratada como linear no sentido de que a saída do filtro é uma combinação linear do conjunto de amostras de entrada. No entanto, o filtro adaptativo é na realidade um dispositivo não linear, uma vez que a adaptação iterativa dos seus parâmetros faz com que sua saída não obedeça ao princípio da superposição. Quando a saída do filtro não é uma simples combinação linear da entrada, (HAYKIN, 2002) o considera como não linear, definição que será seguida ao longo desta dissertação.

O LTE é de implementação relativamente simples (QURESHI, 1985a), mas pode apresentar limitações em situações onde o canal apresenta resposta ao pulso unitário longa, nulos espectrais, não linearidades ou é de fase não mínima (SZCZECINSKI; GEI, 2002). Isso significa que apesar de ser ótimo segundo o MSE, o LTE não é ótimo segundo os critérios ii e iii. Considerando esses critérios, verifica-se que o equalizador ótimo é não linear. De fato, em (FORNEY, 1972) foi demonstrado que o equalizador ótimo, segundo o critério da máxima verossimilhança, é composto por um filtro casado com o canal seguido do algoritmo de Viterbi. Este equalizador é não linear e busca a sequência de máxima verossimilhança, sendo ótimo no sentido de garantir a menor taxa de erros. Além disso, o filtro casado deve ser branqueador para que o ruído do sinal de entrada do algoritmo de Viterbi seja não correlacionado.

Na prática, as soluções ótimas baseadas nos critérios ii e iii, como a proposta por (FORNEY, 1972), são limitadas devido à necessidade de se conhecer o canal. Diante disso, várias estruturas não lineares têm sido estudadas para se obter um equalizador intermediário entre o LTE e os baseados nos critérios ii e iii, como o equalizador de decisão realimentada (decision feedback equalizer - DFE), os filtros baseados em séries de Volterra (BORYS, 2001; MATHEWS; SICURANZA, 2000; ANDRÉ et al., 2013; OGUNFUNMI; DRULLINGER, 2011) e as soluções que utilizam redes neurais (BURSE; YADAV; SHRIVASTAVA, 2010; DAS; PATTNAIK; PADHY, 2014; ZHAO; ZENG; HE, 2011a; ZHAO; ZENG; HE, 2011b).

O DFE, proposto em (AUSTIN, 1967), destaca-se como uma boa alternativa ao LTE, e vem sendo utilizado em sistemas práticos como os de telefonia celular e de TV digital (HOOLE, 1994; GHOSH, 1998; QIN; ZHANG, 2008; ZHANG et al., 2010; KOFIDIS; RONTOGIANNIS, 2010). O DFE é composto por dois filtros, um filtro direto que recebe o sinal degradado e um outro que recebe a realimentação das decisões passadas da saída, compondo um sistema não linear realimentado (PROAKIS; SALEHI, 2007; QURESHI, 1985a). Isso faz do DFE uma solução não linear devido à não linearidade do decisor presente na malha de realimentação. A 
maior limitação desse esquema é o fato de que as decisões erradas resultam em propagação de erros, e a maior vantagem é a sua simplicidade, que permite a implementação de algoritmos de baixa complexidade para a adaptação dos coeficientes em tempo real.

As estruturas baseadas em séries de Volterra e redes neurais apresentam uma alta complexidade computacional, o que inviabiliza a utilização em tempo real. Uma alternativa para a filtragem adaptativa em problemas não lineares é o uso de filtros adaptativos baseados em núcleo (kernel adaptive filters - KAF) (LIU; PRÍNCIPE; HAYKIN, 2010). Os KAFs são capazes de resolver problemas não lineares projetando-se implicitamente o vetor de entrada em um espaço de dimensão maior, onde um filtro adaptativo padrão pode ser utilizado. Os principais problemas do KAF são o crescimento do dicionário, que aumenta o custo computacional, e a escolha adequada da função não linear.

A seguir, o LTE, o DFE, o KAF e seus algoritmos e modos de treinamento são ilustrados.

\subsection{0 equalizador linear transversal}

O equalizador linear transversal nada mais é do que um filtro FIR que em geral é adaptativo (HAYKIN, 2002). A adaptação pode ocorrer de duas maneiras: a supervisionada e a cega. $\mathrm{Na}$ equalização supervisionada usa-se uma sequência de treinamento para obter as estimativas de saída, enquanto na equalização cega usam-se informações estatísticas do sinal transmitido $a(n)$. O esquema da equalização supervisionada com o LTE é mostrado na Figura 4, a seguir.

Figura 4 - Esquema de um sistema de comunicação com equalizador adaptativo supervisionado em fase de treinamento.

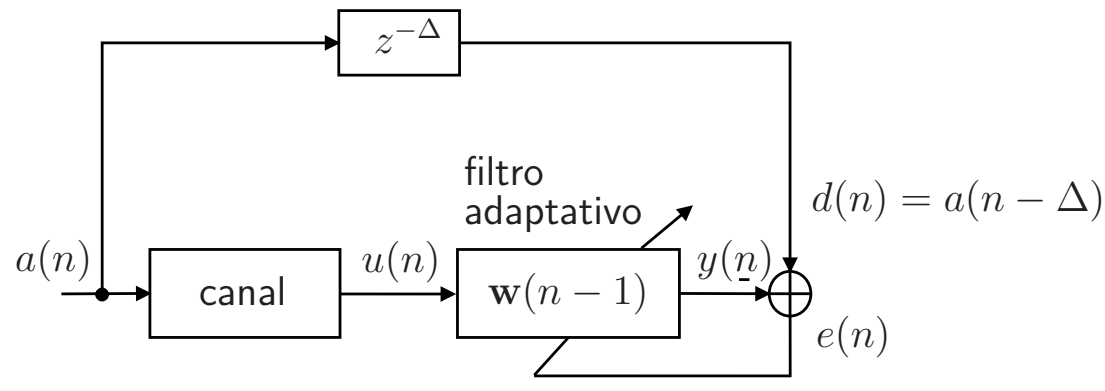

Fonte: Autora.

Considerando como entrada do filtro adaptativo FIR o sinal degradado $u(n)$, a saída $y(n)$ 
corresponde ao produto interno entre o vetor regressor de entrada

$$
\mathbf{u}(n)=\left[\begin{array}{llll}
u(n) & u(n-1) & \cdots & u(n-M+1)
\end{array}\right]^{T}
$$

e o vetor de coeficientes do filtro

$$
\mathbf{w}(n-1)=\left[\begin{array}{llll}
w_{0}(n-1) & w_{1}(n-1) & \cdots & w_{M-1}(n-1)
\end{array}\right]^{T},
$$

isto é

$$
y(n)=\mathbf{u}^{T}(n) \mathbf{w}(n-1),
$$

com $M$ sendo o número de coeficientes do filtro e $(\cdot)^{T}$ o transposto de um vetor. Nesse caso, a sequência de treinamento, conhecida como sinal desejado $d(n)$, corresponde ao sinal transmitido atrasado $a(n-\Delta)$, sendo o erro entre a sequência de treinamento e o sinal de saída dado por

$$
e(n)=a(n-\Delta)-y(n) .
$$

O erro resultante é realimentado a cada iteração com o objetivo de ter seu valor quadrático médio minimizado, obtendo uma saída mais próxima possível de $a(n-\Delta)$. Por usar uma sequência de treinamento, essa configuração do equalizador supervisionado é denominada fase de treinamento. Após a convergência do filtro, a sequência de treinamento deixa de ser usada e é substituída pela estimativa $\hat{a}(n-\Delta)$, saída de um decisor cuja entrada é $y(n)$, conforme mostrado na Figura 5. Essa nova configuração é conhecida como modo de decisão direta (decision directed mode - modo DD) e o erro de decisão é definido por

$$
e_{d}(n)=\hat{a}(n-\Delta)-y(n)
$$

Figura 5 - Esquema de um equalizador adaptativo no modo de decisão direta.

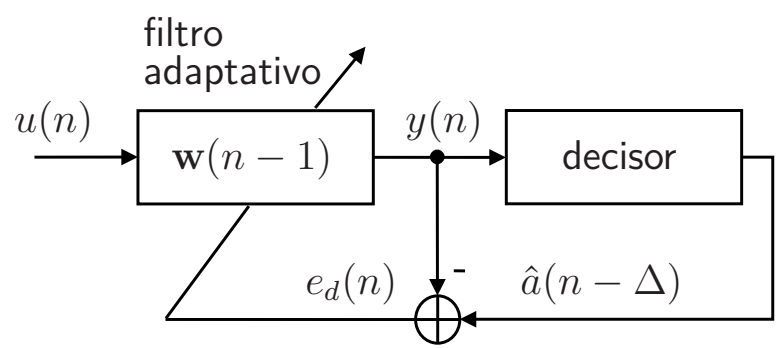

Fonte: Autora.

Cabe observar que é no modo de decisão direta que a informação é efetivamente trans- 
mitida. Além disso, como não se utiliza uma sequência de treinamento, pode-se dizer que o modo de decisão direta é um dos tipos mais rudimentares de equalização cega (QURESHI, 1985b). O equalizador nesse momento é capaz de acompanhar pequenas variações do canal. No entanto, quando o erro quadrático médio passa de um limiar pré-estabelecido, o equalizador volta para o modo de treinamento. Esse chaveamento entre os dois modos sempre ocorre devido às variações do canal (QURESHI, 1985b).

A equalização cega é esquematizada na Figura 6, em que as estatísticas de $a(n)$ e o sinal $y(n)$ são usados pelo algoritmo cego para atualizar os coeficientes do filtro adaptativo por meio do sinal $\varepsilon(n)$.

Figura 6 - Esquema de um equalizador adaptativo cego.

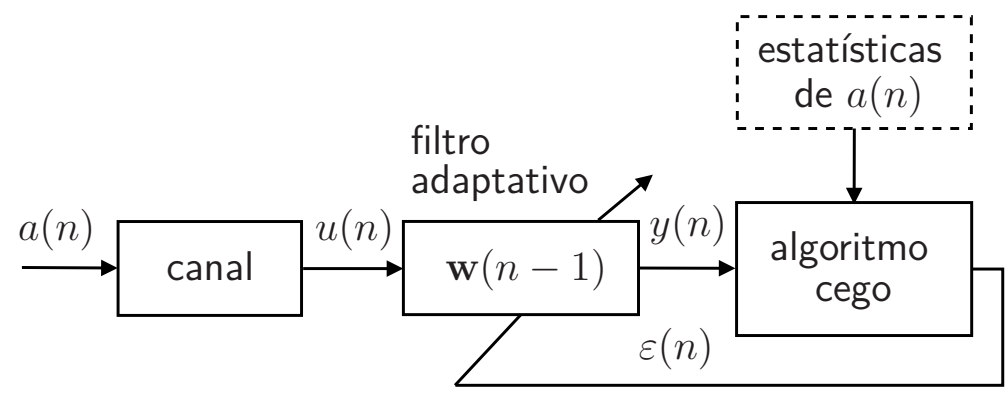

Fonte: Autora.

Existem diversos algoritmos de filtragem adaptativa usados em equalização, dentre eles destacam-se o algoritmo LMS (least mean squares) e o NLMS (normalized least mean squares) para equalização supervisionada e o CMA (constant modulus algorithm) para equalização cega (SAYED, 2008).

O algoritmo LMS executa sucessivas correções nos coeficientes do filtro a fim de minimizar o erro quadrático $e^{2}(n)$, sendo amplamente usado devido a sua simplicidade (HAYKIN, 2002). A atualização dos coeficientes do filtro adaptativo, obtida a partir do método do gradiente estocástico, é dada por

$$
\mathbf{w}(n)=\mathbf{w}(n-1)+\mu e(n) \mathbf{u}(n),
$$

em que $\mu$ é o passo de adaptação e $e(n)$ é definido em (2.2).

Já o algoritmo NLMS apresenta velocidade de convergência maior que a do LMS e pode ser visto como um LMS com passo de adaptação variante no tempo, sendo a atualização dos 
coeficientes definida por (HAYKIN, 2002)

$$
\mathbf{w}(n)=\mathbf{w}(n-1)+\frac{\tilde{\mu}}{\delta+\|\mathbf{u}(n)\|^{2}} e(n) \mathbf{u}(n),
$$

em que $\|\cdot\|$ representa a norma euclidiana, $0<\tilde{\mu}<2$ é o passo de adaptação e $\delta$ é uma constante usada para evitar divisão por zero. Em ambos os casos, o vetor de coeficientes é inicializado com o vetor nulo. Cabe observar que a normalização gerada pela norma quadrática do vetor de entrada possibilita definir o intervalo $] 0,2[$ para a escolha do passo de adaptação $\tilde{\mu}$ no NLMS. Essa normalização é importante principalmente em aplicações onde a potência do sinal de entrada varia no tempo (SAYED, 2008).

Na equalização cega, não se utiliza uma sequência de treinamento e o sinal $y(n)$ é estimado usando estatísticas de ordem superior do sinal $a(n)$. Diferentemente do LMS e do NLMS, que abrangem mais aplicações de filtragem adaptativa, o CMA é específico para equalização de canais de comunicação e precisa conhecer as estatísticas do sinal transmitido para funcionar (GODARD, 1980). O CMA é um algoritmo do tipo gradiente estocástico que minimiza a função custo do módulo constante instantânea, definida como

$$
J_{\mathrm{CMA}}(n)=\left[r-y^{2}(n)\right]^{2}
$$

em que $r$ é um escalar chamado de fator de dispersão e depende das estatísticas do sinal $a(n)$, dado por

$$
r=\frac{\mathrm{E}\left\{a^{4}(n)\right\}}{\mathrm{E}\left\{a^{2}(n)\right\}}
$$

com $\mathrm{E}\{\cdot\}$ indicando a operação esperança matemática. A adaptação dos coeficientes é dada por

$$
\mathbf{w}(n)=\mathbf{w}(n-1)+\mu \varepsilon(n) \mathbf{u}(n),
$$

em que $\varepsilon(n)$ é o "erro de estimação", definido por

$$
\varepsilon(n)=\left[r-y^{2}(n)\right] y(n) .
$$

Diferentemente dos algoritmos de equalização supervisionada, o vetor de coeficientes do CMA não é adaptado quando inicializado com zeros, pois nesse caso o algoritmo converge para um ponto estacionário estável da função custo $(\mathbf{w}=0)$, o que faz com que a saída do filtro fique sempre nula (DING; JOHNSON; KENNEDY, 1992). Em vez disso, costuma-se 
inicializá-lo com o vetor "pino" (DING; LI, 2001), dado por

$$
\mathbf{w}(0)=\left[\begin{array}{lllllll}
0 & \cdots & 0 & 1 & 0 & \cdots & 0
\end{array}\right]^{T} .
$$

A Equação (2.8) indica que o valor de $\varepsilon(n)$ diminui quando $y^{2}(n)$ se aproxima de $r$. Para exemplificar, considera-se $a(n)$ como um sinal 2-PAM, modelado por \pm 1 , em que $r=1,0$. Assim, quando $y(n) \approx \pm 1, y^{2}(n) \approx 1$ e consequentemente $\varepsilon(n) \approx 0$. Em geral, quando $a(n)$ pertence a constelação de módulo não constante, como sinais $N$-PAM em que $N>2$, o sinal $\varepsilon(n)$ não é igual a zero mesmo quando $y(n)$ converge para um dos símbolos da constelação. Dessa forma, o CMA funciona melhor para sinais de constelação de módulo constante apesar de em muitos casos poder conseguir bons resultados de equalização para constelações de módulo não constante (JOHNSON-JR. et al., 1998). Uma alternativa, é o algoritmo RMA (regional multimodulus algorithm, ver mais detalhes no Apêndice A), que é mais eficiente na equalização cega de sinais de módulo não constante que o CMA, além de evitar a divergência e apresentar maior velocidade de convergência (MENDES-FILHO et al., 2009).

Como no caso supervisionado, é comum chavear o equalizador cego para o modo DD quando um determinado limiar de MSE é alcançado na equalização. Uma das dificuldades do chaveamento é a escolha de um limiar de MSE adequado, já que um valor muito grande pode levar a uma convergência ruim ou à não convergência (MAZO, 1980), enquanto um valor muito pequeno pode resultar em um atraso muito grande ou na falha do chaveamento. Em (SILVA; ARENAS-GARCÍA, 2013), mostra-se que é possível realizar o chaveamento entre esses modos por meio de um esquema de combinação convexa entre um equalizador cego e um equalizador DD, onde a escolha de um limiar de MSE não é necessária, sendo o chaveamento portanto automático. No capítulo 3, esse esquema é discutido com mais detalhes.

\subsection{0 equalizador de decisão realimentada}

O equalizador de decisão realimentada é esquematizado na Figura 7 (QURESHI, 1985a; HAYKIN, 2002; PROAKIS; SALEHI, 2007), em que $\mathbf{w}_{f}$ representa o filtro direto e $\mathbf{w}_{b}$ o filtro de realimentação, sendo $M_{f}$ e $M_{b}$ o número de coeficientes de cada filtro, respectivamente. 
Figura 7 - Esquema de um equalizador DFE.

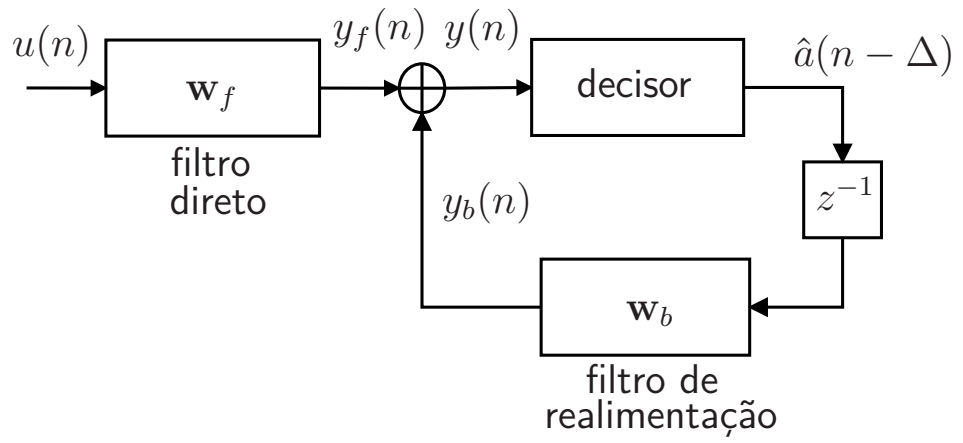

Fonte: Autora.

O filtro direto recebe como sinal de entrada o sinal degradado $u(n)$, de forma que seu vetor regressor de entrada é dado por

$$
\mathbf{u}(n)=\left[\begin{array}{llll}
u(n) & u(n-1) & \cdots & u\left(n-M_{f}+1\right)
\end{array}\right]^{T}
$$

e o vetor de coeficientes do filtro por

$$
\mathbf{w}_{f}=\left[\begin{array}{llll}
w_{f, 0} & w_{f, 1} & \cdots & w_{f, M_{f}-1}
\end{array}\right]^{T},
$$

resultando na saída $y_{f}(n)=\mathbf{u}^{T}(n) \mathbf{w}_{f}$. Já o filtro de realimentação recebe as decisões das estimativas do DFE, com atraso de uma amostra, sendo seu vetor de entrada definido por

$$
\hat{\mathbf{a}}_{\Delta}(n)=\left[\begin{array}{llll}
\hat{a}(n-\Delta-1) & \hat{a}(n-\Delta-2) & \cdots & \hat{a}\left(n-\Delta-M_{b}\right)
\end{array}\right]^{T},
$$

e seu vetor de coeficientes por

$$
\mathbf{w}_{b}=\left[\begin{array}{llll}
w_{b, 0} & w_{b, 1} & \cdots & w_{b, M_{b}-1}
\end{array}\right]^{T},
$$

resultando na saída $y_{b}(n)=\hat{\mathbf{a}}_{\Delta}^{T}(n) \mathbf{w}_{b}$. Assim, a saída do DFE é a soma das saídas de cada filtro, isto é

$$
y(n)=y_{f}(n)+y_{b}(n) .
$$

Os vetores de entrada e os vetores de coeficientes de cada filtro podem ser concatenados em um único vetor, definidos respectivamente por

$$
\mathbf{u}_{f b}(n)=\left[\begin{array}{ll}
\mathbf{u}^{T}(n) & \hat{\mathbf{a}}_{\Delta}^{T}(n)
\end{array}\right]^{T}
$$


e

$$
\mathbf{w}_{f b}=\left[\begin{array}{ll}
\mathbf{w}_{f}^{T} & \mathbf{w}_{b}^{T}
\end{array}\right]^{T},
$$

o que possibilita à Equação (2.13) ser reescrita como

$$
y(n)=\mathbf{u}_{f b}(n)^{T} \mathbf{w}_{f b} .
$$

A presença do decisor na entrada do filtro de realimentação impõe uma não linearidade que faz do DFE uma solução não linear, o que dificulta uma avaliação analítica a respeito do seu desempenho em situações práticas. Diante disso, é comum considerar as decisões corretas e ausência de ruído para a compreensão do comportamento do DFE ou então usar a interpretação clássica de equalizadores como classificadores (GIBSON; COWAN; GRANT, 1990; CHEN et al., 1990; GIBSON; SIU; COWAN, 1991; SILVA, 2001). Dessa forma, verificase o desempenho do DFE superior em relação ao do LTE sobretudo em canais difíceis de equalizar, como os canais que possuem nulos espectrais (SZCZECINSKI; GEI, 2002).

Os vetores de coeficientes dos filtros direto e de realimentação podem ser atualizados de forma supervisionada ou cega. Na equalização supervisionada, pode-se utilizar, por exemplo, os algoritmos LMS ou NLMS para atualizar os coeficientes do vetor $\mathbf{w}_{f b}$. Na equalização cega, algoritmos baseados no critério de módulo constante, como o CMA, podem resultar em soluções degeneradas, isto é, quando a saída do equalizador deixa de depender da sua entrada (SZCZECINSKI; GEI, 2002; PAPADIAS; PAULRAJ, 1995). Para evitar estas soluções, foram propostos os algoritmos do gradiente estocástico DFE-CMA-FF (PAPADIAS; PAULRAJ, 1995) e DFE-CMA-FB (SZCZECINSKI; GEI, 2002), obtidos com a imposição de restrições no critério do módulo constante. No primeiro algoritmo a restrição é incluída apenas no filtro direto e no segundo ela é incluída no direto e no de realimentação, o que levou a um melhor desempenho.

\subsection{0 equalizador com filtro adaptativo baseado em kernel}

Dado que o LTE não funciona bem em canais de comunicação não lineares e o DFE pode apresentar um desempenho limitado nesses canais devido à realimentação de decisões erradas, o emprego de filtros adaptativos baseados em kernel representa uma alternativa ao problema. Os KAFs realizam a filtragem adaptativa projetando as entradas em um espaço de alta dimensão, onde o problema não linear pode ser solucionado de forma linear (LIU; PRÍNCIPE; HAYKIN, 2010). Esta solução apresenta melhor desempenho para problemas não lineares que as versões 
de filtros adaptativos convencionais, tendo sido aplicada principalmente em identificação de sistemas (PARREIRA et al., 2012), cancelamento de eco (TOBAR; KUNG; MANDIC, 2014), predição de séries temporais (LIU; PRÍNCIPE; HAYKIN, 2010; TOBAR; KUNG; MANDIC, 2014; VAERENBERGH; AZPICUETA-RUIZ; COMMINIELLO, 2016), e equalização de canais (LIU; PRÍNCIPE; HAYKIN, 2010). A seguir, são apresentados o algoritmo KLMS (kernel least mean squares), versão "kernelizada" do LMS, e o algoritmo QKLMS (quantized kernel least mean squares), versão do KLMS com menor custo computacional.

\subsubsection{O algoritmo KLMS}

Assim como o LMS para filtragem adaptativa tradicional, o KLMS é o algoritmo mais popular para filtragem adaptativa com kernel. No KLMS, os vetores de entrada $\mathbf{u}(n) \in \mathbb{R}^{M}$, são mapeados em $\Phi(\mathbf{u}(n)) \in \mathbb{F}$, em que $\mathbb{F}$ é denominado "espaço das características de alta dimensão". Segundo (LIU; PRÍNCIPE; HAYKIN, 2010), o kernel deve ser uma função contínua, simétrica e positiva definida $\kappa: \mathbb{R}^{M} \times \mathbb{R}^{M} \rightarrow \mathbb{F}$, tal que vale

$$
\kappa\left(\mathbf{u}, \mathbf{u}^{\prime}\right)=\Phi(\mathbf{u})^{T} \Phi\left(\mathbf{u}^{\prime}\right)
$$

Na literatura a função $\kappa(\cdot, \cdot)$ que satisfaz essas propriedades é conhecida como kernel de Mercer e a Equação (2.17) como truque do kernel. O "truque" ocorre porque o produto interno dos vetores $\Phi(\mathbf{u})$ e $\Phi\left(\mathbf{u}^{\prime}\right)$ pode ser calculado sem conhecer explicitamente a função $\Phi(\cdot)$, bastando apenas conhecer o kernel $\kappa(\cdot, \cdot)$. A Figura 8 esquematiza a filtragem adaptativa com o kernel para a equalização supervisionada, em que no espaço $\mathbb{F}$, o algoritmo LMS é usado para adaptar o vetor de coeficientes do filtro, representado por $\Omega(n)$. O objetivo do KAF é estimar o sinal desejado $d(n) \in \mathbb{R}$, utilizando o mapeamento do vetor de entrada $\mathbf{u}(n)$ no espaço $\mathbb{F}$.

O algoritmo KLMS pode ser resumido pelas seguintes equações:

$$
\begin{aligned}
\boldsymbol{\Omega}(0) & =\mathbf{0} \\
e(n) & =d(n)-\Phi(\mathbf{u}(n))^{T} \boldsymbol{\Omega}(n-1), \\
\boldsymbol{\Omega}(n) & =\boldsymbol{\Omega}(n-1)+\mu e(n) \Phi(\mathbf{u}(n)),
\end{aligned}
$$

em que $\mu$ é o passo de adaptação e $d(n)=a(n-\Delta)$. Por meio de manipulações algébricas 
simples, pode-se escrever o vetor de coeficientes como

$$
\boldsymbol{\Omega}(n)=\sum_{i=1}^{n} \mu e(i) \Phi(\mathbf{u}(i))
$$

e usando (2.17), a saída do filtro pode ser escrita, por sua vez, como

$$
y(n)=\Phi(\mathbf{u}(n))^{T} \boldsymbol{\Omega}(n-1)=\sum_{i=1}^{n-1} \underbrace{\mu e(i)}_{b_{i}(n-1)} \kappa(\mathbf{u}(n), \mathbf{u}(i)),
$$

em que $b_{i}(n-1)$ é o coeficiente que pondera o kernel $\kappa(\mathbf{u}(n), \mathbf{u}(i))$. De (2.22), pode-se notar que o "truque" do kernel elimina a necessidade do cálculo dos coeficientes $\Omega(n)$ do algoritmo (SCHÖLKOPF; SMOLA, 2002).

Figura 8 - Esquema de um filtro adaptativo baseado em kernel aplicado à equalização supervisionada.

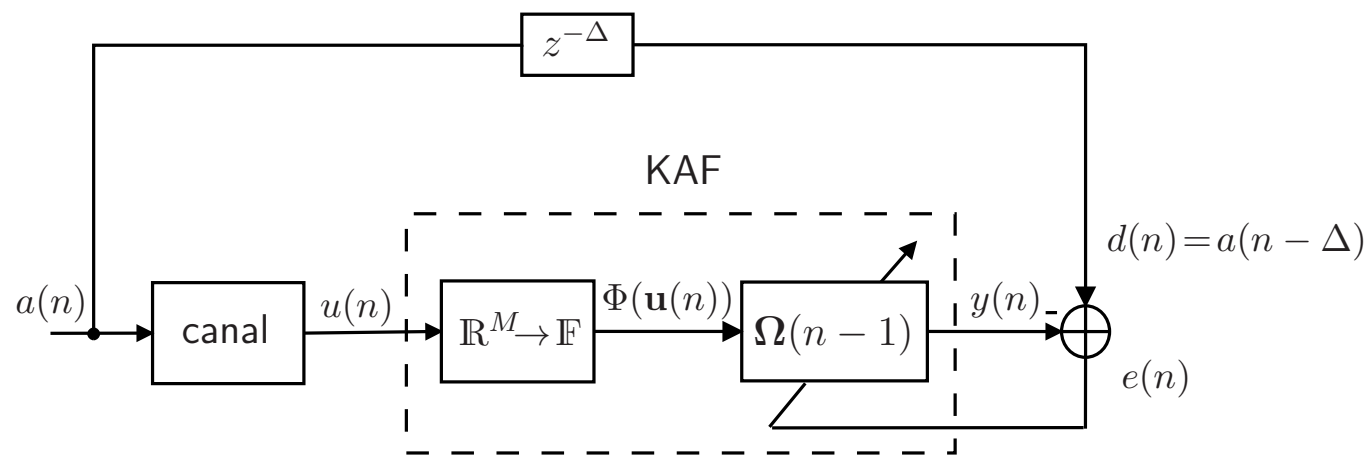

Fonte: Autora.

O kernel mais utilizado na literatura é o gaussiano, definido como

$$
\kappa\left(\mathbf{u}, \mathbf{u}^{\prime}\right)=\exp \left(-\frac{\left\|\mathbf{u}-\mathbf{u}^{\prime}\right\|^{2}}{2 \sigma^{2}}\right)
$$

sendo $\sigma$ a largura do kernel (LIU; PRÍNCIPE; HAYKIN, 2010). Cabe destacar que outros tipos de kernel também podem ser utilizados no KLMS, mas neste trabalho utiliza-se apenas o gaussiano.

As equações que implementam o algoritmo KLMS, para $N$ iterações, estão resumidas na Tabela 1. Nessa tabela, b representa o vetor de coeficientes que pondera o kernel no somatório (2.22), sendo $b_{i}(n)$ o $i$-ésimo elemento desse vetor na $n$-ésima iteração. Cada vetor de entrada $\mathbf{u}(n)$ é armazenado na matriz $\mathbf{C}(n)$, denominada dicionário do algoritmo. Pode-se notar que para calcular $y(n)$, o KLMS deve calcular $\kappa(\mathbf{u}(n), \mathbf{u}(i))$, para $i=1 \ldots, n-1$. 
Dessa forma, todos os vetores de entrada $\mathbf{u}(i)$ ficam armazenados no dicionário $\mathbf{C}(n)$, que tem cardinalidade $N_{c}(n)=n-1$.

Tabela 1: Sumário do algoritmo KLMS.

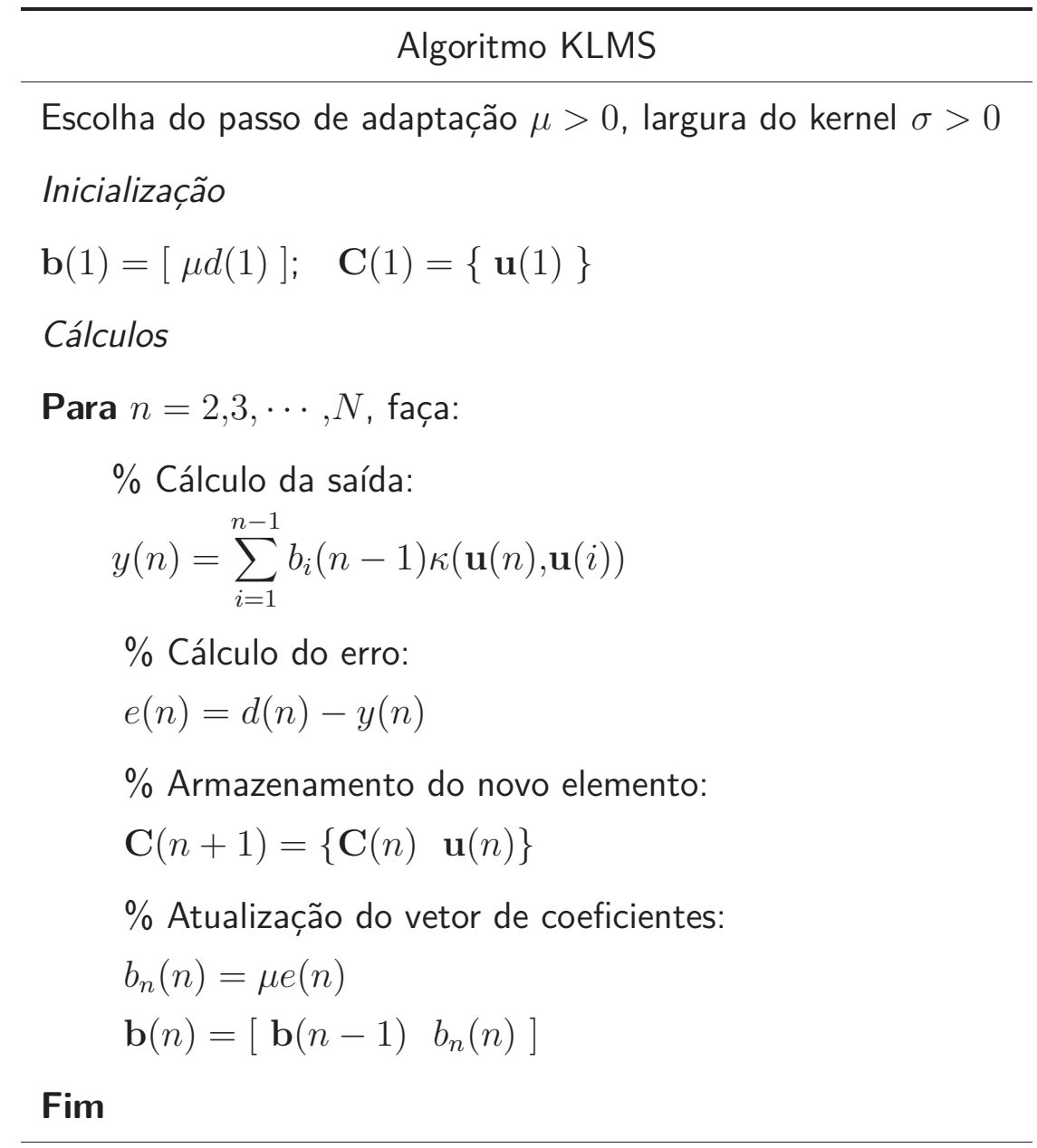

Fonte: Autora.

A quantidade de vetores armazenados no dicionário $\mathbf{C}$ é igual ao número de coeficientes do vetor $\mathbf{b}$. Uma vez que os tamanhos do dicionário $N_{c}(n)=n-1$ e do vetor $\mathbf{b}$ aumentam linearmente com as iterações, o tempo de execução do algoritmo também aumenta, dificultando a sua implementação em aplicações em tempo real. Para contornar esse problema, diferentes técnicas de esparsificação foram propostas na literatura para reduzir o tamanho do dicionário (PLATT, 1991; RICHARD; BERMUDEZ; HONEINE, 2009). Uma dessas técnicas é o critério da novidade (PLATT, 1991), que estabelece que um vetor de entrada só é adicionado ao dicionário se a sua distância em relação aos demais vetores for menor que um limiar predefindo. 
Um algoritmo que incorpora o critério da novidade ao KLMS é revisitado a seguir.

\subsubsection{O algoritmo QKLMS}

O algoritmo QKLMS, proposto em (CHEN et al., 2012), é uma alternativa às técnicas de esparsificação, sendo similar ao algoritmo KLMS com critério de novidade. Inicialmente, definese o índice $c_{i} \in\{1,2, \ldots n-1\}$ para distinguir os elementos do dicionário $\mathbf{u}\left(c_{1}\right), \ldots, \mathbf{u}\left(c_{N_{c}(n)}\right)$ do vetor de entrada $\mathbf{u}(n)$. Utilizando essa notação para decidir se o vetor de entrada deve ou não ser acrescentado ao dicionário, define-se

$$
\operatorname{dis}(\mathbf{u}(n), \mathbf{C}(n))=\min _{1 \leq i \leq N_{c}(n)}\left\|\mathbf{u}(n)-\mathbf{u}\left(c_{i}\right)\right\|
$$

Se $\operatorname{dis}(\mathbf{u}(n), \mathbf{C}(n)) \leq \rho$, sendo $\rho$ um limiar, mantém-se o dicionário inalterado e atualiza-se o coeficiente do vetor mais próximo como

$$
b_{i^{*}}(n)=b_{i^{*}}(n-1)+\mu e(n),
$$

em que

$$
i^{*}=\arg \min _{1 \leq i \leq N_{c}(n)}\left\|\mathbf{u}(n)-\mathbf{u}\left(c_{i}\right)\right\|
$$

Caso contrário, acrescenta-se o vetor de entrada ao dicionário e seu respectivo coeficiente deve ser guardado na memória, i.e.,

$$
\mathbf{C}(n+1)=\{\mathbf{C}(n) \quad \mathbf{u}(n)\}
$$

e

$$
\mathbf{b}(n)=\left[\begin{array}{ll}
\mathbf{b}(n-1) & \mu e(n)
\end{array}\right]
$$

A diferença do QKLMS em relação ao KLMS que usa o critério da novidade está na atualização (2.25). As equações que implementam o QKLMS são mostradas na Tabela 2. Devido às suas vantagens inerentes, apenas o QKLMS será usado nos capítulos seguintes desta dissertação. Por fim, cabe observar que na literatura não há uma versão cega do algoritmo KLMS. 
Tabela 2: Sumário do algoritmo QKLMS.

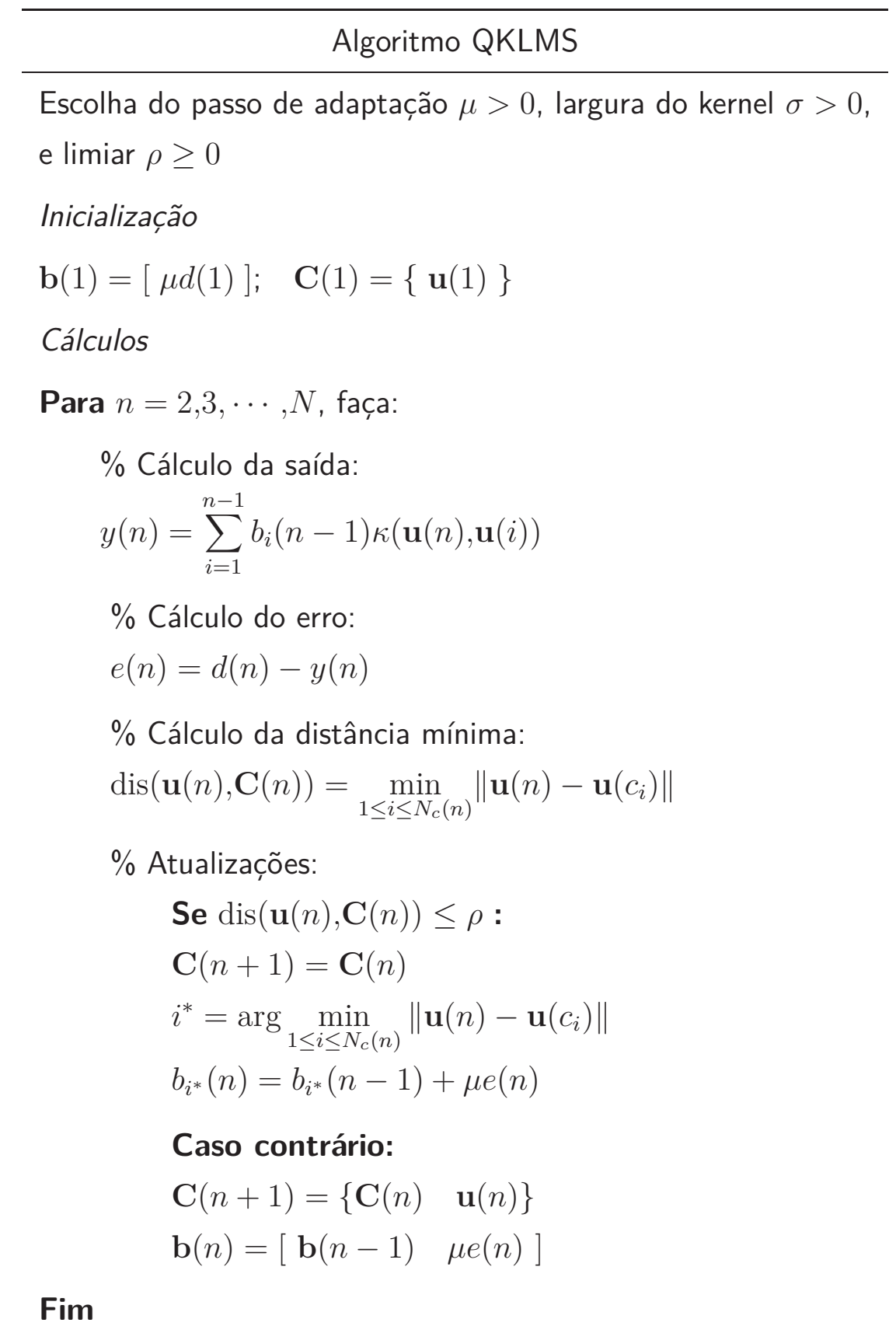

Fonte: Autora.

\subsection{Conclusão}

Neste capítulo, a equalização com filtros adaptativos foi abordada considerando o LTE, o DFE e o KAF. Além disso, discutiram-se os modos de adaptação supervisionada, cega e o modo de decisão direta. Os algoritmos supervisionados LMS, NLMS, KLMS e QKLMS para 
equalização foram revisitados. No modo cego, considerou-se o CMA para a adaptação de um LTE. A escolha do equalizador depende do cenário. Se o canal for linear e não tiver nulos espectrais, o LTE pode dar bons resultados. No entanto se o canal tiver nulos espectrais, é mais adequando usar o DFE. Por fim, se o canal possuir não linearidades, o KAF pode ser uma alternativa. Para evitar a transmissão de uma sequência de treinamento, a equalização deve ser cega. A questão que fica é como utilizar um equalizador cego em canais não lineares que ainda apresente um bom desempenho e possa ser chaveado para o modo DD? O chaveamento entre o modo de treinamento supervisionado ou modo cego para o modo DD depende de um limiar de MSE pré-estabelecido. A determinação desse limiar depende da constelação, razão sinal-ruído e do canal, o que torna o chaveamento dependente do cenário. Uma solução para esse problema é a que utiliza um chaveamento automático. No capítulo 3 serão abordadas soluções automáticas para esse chaveamento baseando-se na combinação de filtros adaptativos considerando as soluções lineares e não lineares deste capítulo. 


\section{COMBINAÇÃO CONVEXA DE FILTROS ADAPTATIVOS PARA EQUALIZAÇÃO CEGA}

Este capítulo trata do esquema de equalização adaptativa cega baseado na combinação convexa entre um equalizador cego e um equalizador no modo de decisão direta. Na Seção 3.1, esse esquema é apresentado levando-se em conta o algoritmo CMA para o equalizador cego e o algoritmo NLMS para o equalizador no modo DD, juntamente com os resultados de algumas simulações. Na Seção 3.2, esse esquema de combinação é estendido considerando soluções não lineares para o equalizador no modo DD, como o equalizador de decisão realimentada e o filtro adaptativo baseado em kernel. Esses novos esquemas podem ser úteis para a equalização cega de canais difíceis de equalizar, como os canais com nulos espectrais e os não lineares. Na mesma seção, são mostrados os resultados de simulações que verificam o comportamento dessas soluções para sinais reais do tipo PAM. Por fim, na Seção 3.3, são feitas as considerações finais sobre o capítulo.

\subsection{Combinação convexa entre equalizadores cegos e equalizadores no modo DD}

A combinação convexa entre dois ou mais filtros adaptativos é um esquema em que as saídas de cada filtro são ponderadas conforme seu desempenho e combinadas para gerar uma saída que proporcione um desempenho global melhor ou igual ao de cada filtro individual (ARENAS-GARCÍA; GÓMEZ-VERDEJO; FIGUEIRAS-VIDAL, 2005; ARENAS-GARCÍA; FIGUEIRAS-VIDAL; SAYED, 2006; SILVA; ARENAS-GARCÍA, 2013). A combinação pode funcionar de maneira supervisionada ou cega. Na combinação supervisionada, os filtros componentes são supervisionados. Na combinação cega, os filtros componentes podem ser ambos cegos, ou um cego e o outro no modo de decisão direta.

A Figura 9 mostra o esquema da combinação convexa de um equalizador CMA com um equalizador NLMS no modo DD, denotado por NLMS-DD. Esse esquema também funciona para outras combinações de algoritmos, como é mostrado em (SILVA; ARENAS-GARCÍA, 2013). A saída global $y(n)$ é obtida por meio da combinação das saídas $y_{1}(n)$ e $y_{2}(n)$ dos filtros individuais, isto é

$$
y(n)=\lambda(n) y_{1}(n)+[1-\lambda(n)] y_{2}(n)
$$

em que $\lambda(n) \in[0,1]$ é o parâmetro de mistura e $y_{i}(n)=\mathbf{u}^{T}(n) \mathbf{w}_{i}(n-1)$ são as saídas dos equalizadores cego e DD, respectivamente para $i=1,2$ (SILVA; ARENAS-GARCÍA, 2013). A saída global $y(n)$ passa por um decisor, resultando no sinal decidido $\hat{a}(n-\Delta)$, que faz 
papel de sinal desejado, sendo usado para calcular o erro do filtro NLMS-DD. Os vetores de coeficientes de cada filtro $\mathbf{w}_{1}(n-1)$ e $\mathbf{w}_{2}(n-1)$ são adaptados com seus respectivos erros $\varepsilon_{1}(n)$ e $e_{d 2}(n)$, isto é

$$
\begin{aligned}
& \mathbf{w}_{1}(n)=\mathbf{w}_{1}(n-1)+\mu_{1} \varepsilon_{1}(n) \mathbf{u}(n), \\
& \mathbf{w}_{2}(n)=\mathbf{w}_{2}(n-1)+\frac{\mu_{2}}{\delta+\|\mathbf{u}(n)\|^{2}} e_{d 2}(n) \mathbf{u}(n),
\end{aligned}
$$

sendo

$$
\begin{aligned}
\varepsilon_{1}(n) & =\left[r-y_{1}^{2}(n)\right] y_{1}(n), \\
e_{d 2}(n) & =\hat{a}(n-\Delta)-y_{2}(n) .
\end{aligned}
$$

Figura 9 - Combinação convexa entre um equalizador CMA e um NLMS-DD, $\operatorname{com} \lambda(n)$ obtido a partir da minimização de $J_{\mathrm{CMA}}(n)$.

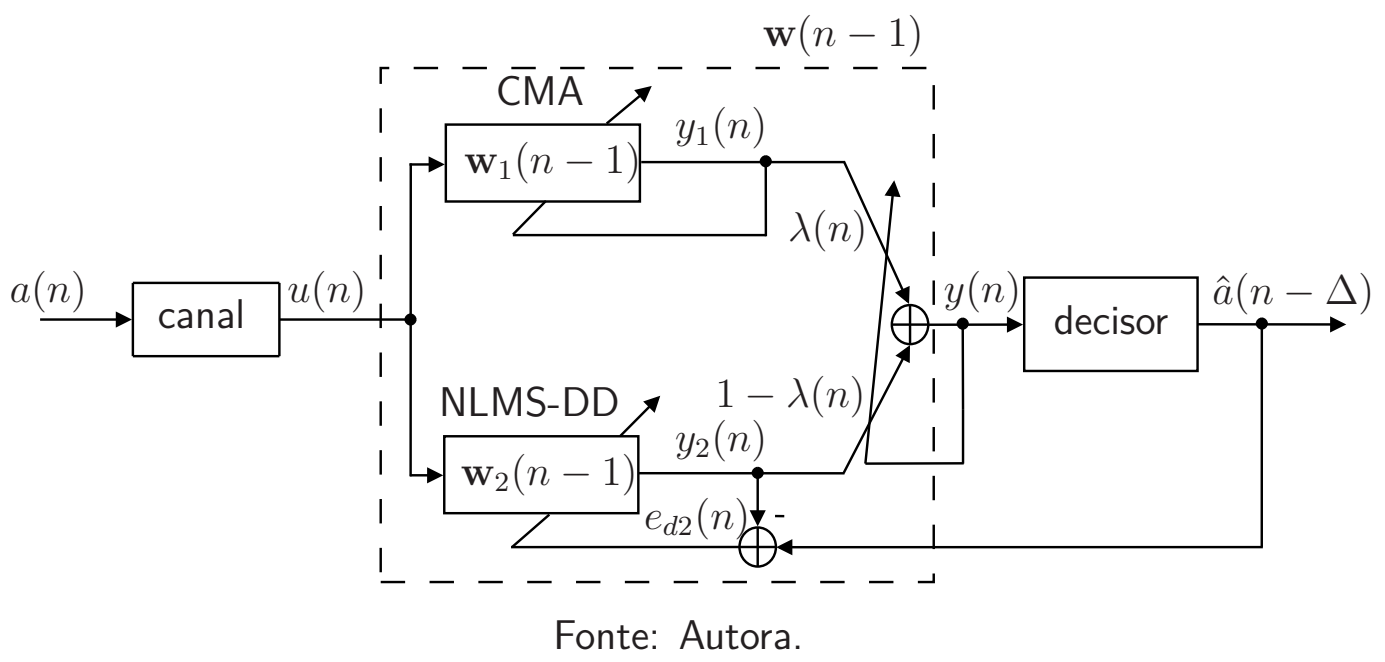

No início das iterações, as estimativas $\hat{a}(n-\Delta)$ são ruins e o NLMS-DD apresenta desempenho inferior ao do CMA, de maneira que $y(n)$ se aproxima da saída do CMA e $\lambda \approx 1$. Conforme o CMA converge, as estimativas $\hat{a}(n-\Delta)$ melhoram, consequentemente $\circ$ desempenho do NLMS-DD também melhora e $y(n)$ se aproxima da saída do NLMS-DD, pois $\lambda \approx 0$. Dessa forma, diferentemente dos esquemas de combinação comumente propostos na literatura (e.g., (ARENAS-GARCÍA; FIGUEIRAS-VIDAL; SAYED, 2006) e suas referências), os filtros do esquema da Figura 9 não são desacoplados, pois o bom funcionamento do NLMSDD depende da qualidade da estimativa $\hat{a}(n-\Delta)$, que por sua vez, depende do desempenho do CMA.

Para que a combinação tenha um bom comportamento, é importante que os filtros componentes sejam adaptados de acordo com suas próprias regras. O parâmetro de mistura $\lambda(n)$, por sua vez, deve ser adaptado para se obter um bom desempenho global 
(ARENAS-GARCÍA; FIGUEIRAS-VIDAL; SAYED, 2006). A fim de restringir esse parâmetro ao intervalo $[0,1]$ e reduzir o ruído de gradiente quando $\lambda \approx 0$ e $\lambda \approx 1$, uma transformação não linear e uma variável auxiliar $\alpha(n)$ são usadas, ou seja

$$
\lambda(n)=\varphi[\alpha(n-1)]=\frac{\operatorname{sgm}[\alpha(n-1)]-\operatorname{sgm}\left[-\alpha^{+}\right]}{\operatorname{sgm}\left[\alpha^{+}\right]-\operatorname{sgm}\left[-\alpha^{+}\right]},
$$

sendo

$$
\operatorname{sgm}[\alpha(n-1)]=\frac{1}{1+e^{-\alpha(n-1)}}
$$

a função sigmoidal, que está representada na Figura 10 juntamente com a função de ativação $\varphi[\cdot]$, proposta em (LÁZARO-GREDILLA et al., 2010). A função $\operatorname{sgm}[\alpha(n-1)]$ é igual a zero quando $\alpha(n-1) \ll 0$ e igual a um quando $\alpha(n-1) \gg 0$. Já a função $\varphi[\cdot]$ é uma versão escalonada e deslocada da função sigmoidal e atinge valor um quando $\alpha(n-1)=\alpha^{+}$e zero quando $\alpha(n-1)=-\alpha^{+}$.

Figura 10 - Função sigmoidal $\operatorname{sgm}[\alpha(n-1)]$ e função de ativação $\varphi[\alpha(n-1)]$, para $\alpha^{+}=4$.
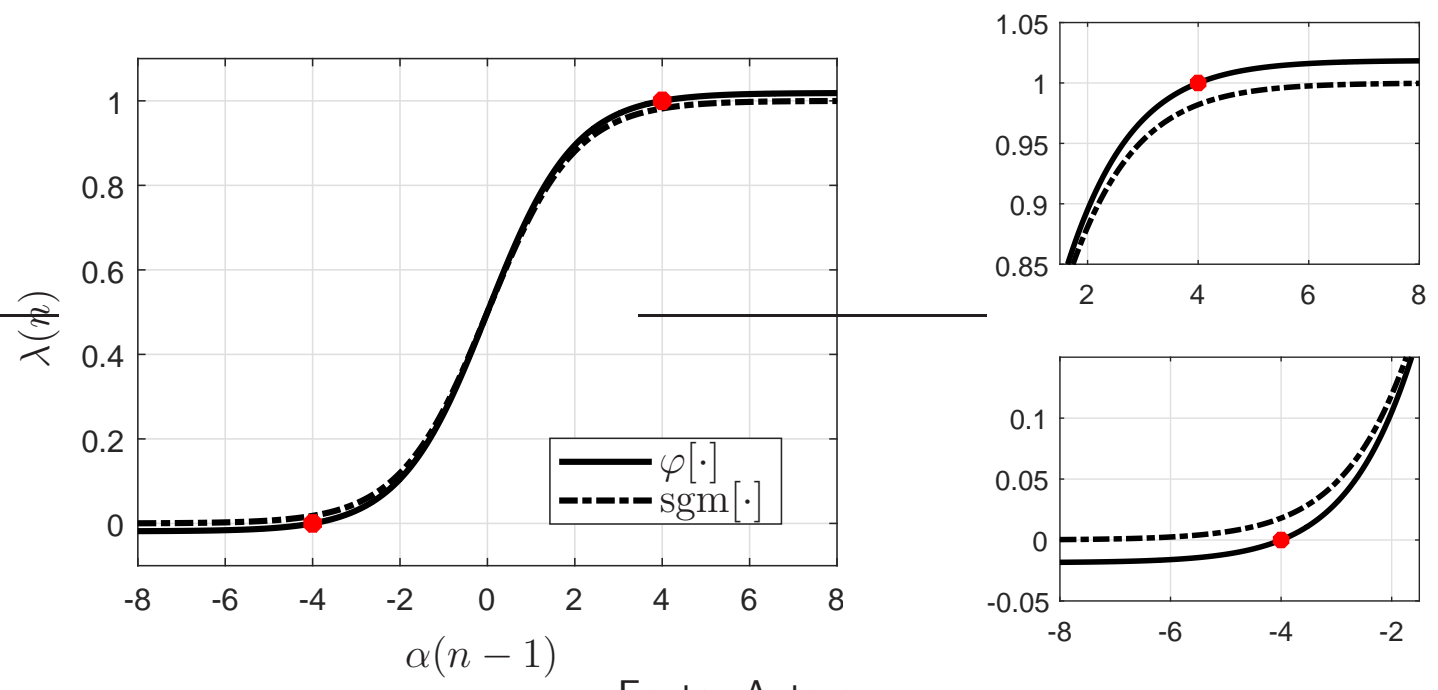

Fonte: Autora.

Dessa forma, a atualização do parâmetro de mistura $\lambda(n)$ ocorre por meio da atualização da variável auxiliar $\alpha(n-1)$, cuja equação de adaptação é obtida utilizando o método do gradiente estocástico para minimizar uma função custo instantânea relacionada à saída global $y(n)$. A seguir, são discutidos os esquemas de combinação que minimizam a função custo instantânea do módulo constante e o erro quadrático médio de decisão. 


\subsubsection{Combinação com minimização da função custo do módulo constante}

A minimização da função custo instantânea de módulo constante $J_{\mathrm{CMA}}(n)$, definida na Equação (2.6) (página 13), resulta na seguinte equação de atualização para a variável auxiliar (SILVA; ARENAS-GARCÍA, 2013)

$$
\alpha(n)=\alpha(n-1)+\mu_{\alpha} \varepsilon(n)\left[y_{1}(n)-y_{2}(n)\right] \varphi^{\prime}[\alpha(n-1)],
$$

em que $\mu_{\alpha}$ é o passo de adaptação e $\varepsilon(n)=\left[r-y^{2}(n)\right] y(n)$ é o "erro de estimação" global, sendo

$$
\varphi^{\prime}[\alpha(n-1)]=\frac{d \lambda(n)}{d \alpha(n-1)}=\frac{\operatorname{sgm}[\alpha(n-1)]\{1-\operatorname{sgm}[\alpha(n-1)]\}}{\operatorname{sgm}\left[\alpha^{+}\right]-\operatorname{sgm}\left[-\alpha^{+}\right]} .
$$

A variável auxiliar $\alpha(n)$ deve ser restrita por saturação ao intervalo $\left[-\alpha^{+}, \alpha^{+}\right]$, pois quando $|\alpha(n)|$ é muito grande, o termo $\varphi^{\prime}[\alpha(n-1)]$ torna-se nulo e a adaptação deixa de acontecer. Uma escolha comum na literatura é $\alpha^{+}=4$ (ver, e.g., (ARENAS-GARCÍA; FIGUEIRAS-VIDAL; SAYED, 2006; LÁZARO-GREDILLA et al., 2010)), usado também na Figura 10. Cabe observar que a função $\varphi[\alpha(n-1)]$ extrapola o intervalo $[0,1]$ quando $|\alpha(n)|>4$, o que, em outras palavras, justifica a necessidade da restrição de $\alpha(n)$ ao intervalo $[-4,4]$.

Normalmente, o valor de $\mu_{\alpha}$ depende das características do cenário (degradação do canal, razão sinal-ruído, etc.), o que pode dificultar sua escolha. Para garantir um comportamento mais robusto a mudanças do ambiente, utiliza-se um esquema de normalização do passo, proposto em (AZPICUETA-RUIZ; FIGUEIRAS-VIDAL; ARENAS-GARCÍA, 2008), que leva à seguinte atualização

$$
\alpha(n)=\alpha(n-1)+\frac{\mu_{\alpha}}{p(n)} \varepsilon(n)\left[y_{1}(n)-y_{2}(n)\right] \varphi^{\prime}[\alpha(n-1)],
$$

em que

$$
p(n)=\eta p(n-1)+(1-\eta)\left[y_{1}(n)-y_{2}(n)\right]^{2},
$$

sendo $0 \ll \eta<1$, um fator de esquecimento normalmente escolhido como $\eta=0,9$ (AZPICUETA-RUIZ; FIGUEIRAS-VIDAL; ARENAS-GARCÍA, 2008).

\subsubsection{Combinação com minimização do erro quadrático médio de decisão}

Nesse esquema, mostrado na Figura 11, a variável auxiliar $\alpha(n)$ é adaptada com o objetivo de minimizar o erro de decisão global ao quadrado,

$$
e_{d}^{2}(n)=[\hat{a}(n-\Delta)-y(n)]^{2},
$$


que resulta em uma equação de adaptação similar à Equação (3.10), dada por

$$
\alpha(n)=\alpha(n-1)+\frac{\mu_{\alpha}}{p(n)} e_{d}(n)\left[y_{1}(n)-y_{2}(n)\right] \varphi^{\prime}[\alpha(n-1)]
$$

em que $\mu_{\alpha}$ é o passo de adaptação e $p(n)$ é definido em (3.11).

Figura 11 - Combinação convexa de um equalizador CMA com um NLMS-DD, com $\lambda(n)$ adaptado a partir da minimização de $e_{d}^{2}(n)$.

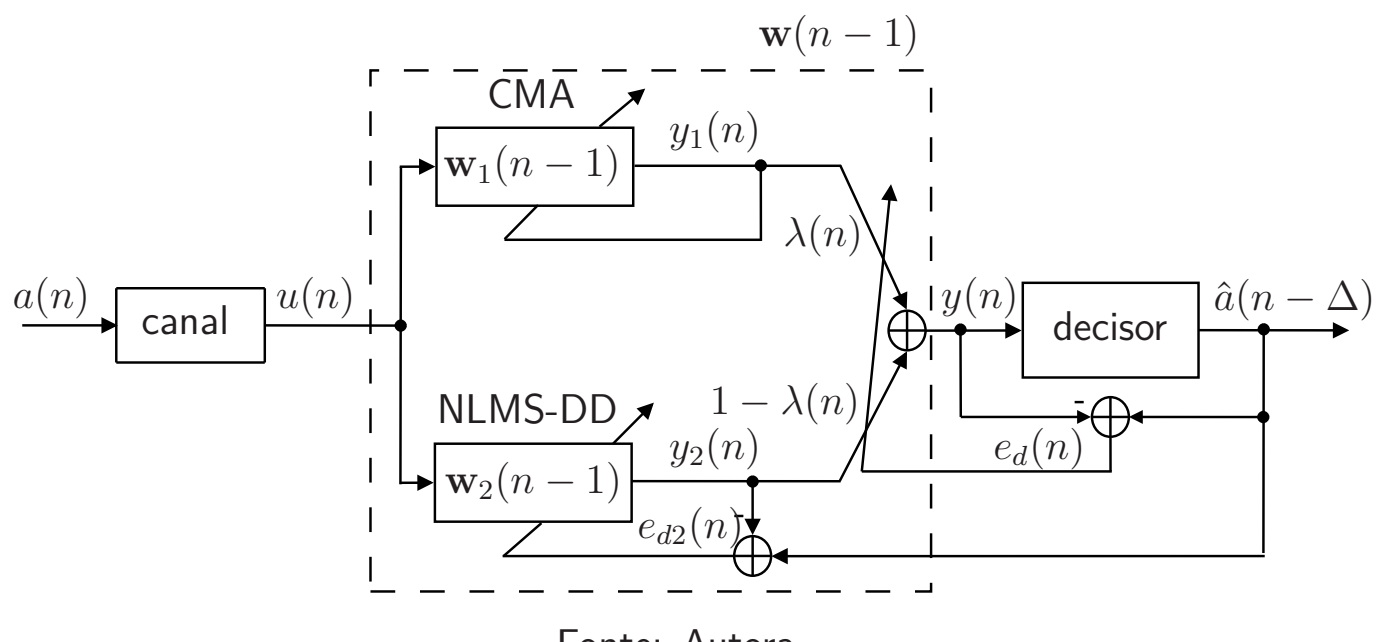

Fonte: Autora.

Segundo (SILVA; ARENAS-GARCÍA, 2013), os esquemas de combinação discutidos, quando aplicados em sinais QAM (quadrature amplitude modulation), apresentam desempenho semelhante em termos de MSE. No entanto, em termos de taxa de erro de símbolo (symbol error rate - SER), a combinação que utiliza o erro do CMA $\varepsilon(n)$ na adaptação de $\alpha(n)$ apresentou desempenho melhor que a combinação que utiliza o erro de decisão $e_{d}(n)$. Além disso, quando $e_{d}(n)$ é usado, o passo de adaptação $\mu_{\alpha}$ precisa ser elevado para que a combinação gere bons resultados. Essa escolha provoca em $\lambda(n)$ um comportamento oscilatório entre zero e um quando o algoritmo cego domina a combinação, convergindo para zero quando o esquema é chaveado para o modo de decisão direta.

Para simplificar, os esquemas discutidos acima serão tratados nesta dissertação como combinação convexa com o erro do CMA $\varepsilon(n)$ e combinação convexa com erro de decisão $e_{d}(n)$. A Tabela 3 mostra as equações que implementam o algoritmo de combinação convexa com erro de decisão $e_{d}(n)$ entre o CMA e o NLMS-DD. Esse mesmo algoritmo pode ser utilizado para a combinação com o erro do CMA, substituindo $e_{d}(n)$ por $\varepsilon(n)$ na atualização da variável auxiliar $\alpha(n)$. Na inicialização do algoritmo, define-se $\alpha(0)=\alpha^{+}$para que $\lambda(1)=1$ e a combinação comece chaveada para o CMA, já que é certo que o seu desempenho é o melhor 
no início das iterações. A função $\operatorname{sign}[\cdot]$ usada no algoritmo é definida por

$$
\operatorname{sign}(x)=\left\{\begin{array}{ll}
1, & x \geq 0 \\
-1, & x<0
\end{array} .\right.
$$

Tabela 3: Sumário do algoritmo de combinação convexa entre o CMA e o NLMS-DD, com minimização do erro de decisão global ao quadrado $e_{d}^{2}(n)$.

\section{Combinação convexa entre o CMA e o NLMS-DD}

Escolha dos passos de adaptação $\mu_{1}, \mu_{2}>0 ; \quad r=\frac{\mathrm{E}\left\{a^{4}(n)\right\}}{\mathrm{E}\left\{a^{2}(n)\right\}}$

Inicialização

$\mathbf{w}_{1}(0)=\left[\begin{array}{lllllll}0 & \cdots & 0 & 1 & 0 & \cdots & 0\end{array}\right]^{T} ; \quad \mathbf{w}_{2}(0)=\mathbf{0}_{M}$

$\alpha(0)=\alpha^{+} ; \quad p(0)=1 ; \quad \eta=0,9$

\section{Cálculos}

Para $n=1,2, \cdots, N$, faça:

\% Cálculo das saídas:

$$
\begin{aligned}
& y_{1}(n)=\mathbf{u}^{T}(n) \mathbf{w}_{1}(n-1) \\
& y_{2}(n)=\mathbf{u}^{T}(n) \mathbf{w}_{2}(n-1)
\end{aligned}
$$

Se $|\alpha(n-1)|>\alpha^{+}: \quad \alpha(n-1) \leftarrow \alpha^{+} \operatorname{sign}[\alpha(n-1)]$

$\lambda(n)=\varphi[\alpha(n-1)]=\frac{\operatorname{sgm}[\alpha(n-1)]-\operatorname{sgm}\left[-\alpha^{+}\right]}{\operatorname{sgm}\left[\alpha^{+}\right]-\operatorname{sgm}\left[-\alpha^{+}\right]}$

$y(n)=\lambda(n) y_{1}(n)+[1-\lambda(n)] y_{2}(n)$

\% Cálculo dos erros:

$\varepsilon_{1}(n)=\left[r-y_{1}^{2}(n)\right] y_{1}(n)$

$\hat{a}(n-\Delta)=\operatorname{decisor}[y(n)]$

$e_{d 2}(n)=\hat{a}(n-\Delta)-y_{2}(n)$

$e_{d}(n)=\hat{a}(n-\Delta)-y(n)$

\% Atualizações: 


$$
\begin{aligned}
& \text { Combinação convexa entre o CMA e o NLMS-DD } \\
& \mathbf{w}_{1}(n)=\mathbf{w}_{1}(n-1)+\mu_{1} \varepsilon_{1}(n) \mathbf{u}(n) \\
& \mathbf{w}_{2}(n)=\mathbf{w}_{2}(n-1)+\frac{\mu_{2}}{\delta+\|\mathbf{u}(n)\|^{2}} e_{d 2}(n) \mathbf{u}(n) \\
& \varphi^{\prime}[\alpha(n-1)]=\frac{\operatorname{sgm}[\alpha(n-1)]\{1-\operatorname{sgm}[\alpha(n-1)]\}}{\operatorname{sgm}\left[\alpha^{+}\right]-\operatorname{sgm}\left[-\alpha^{+}\right]} \\
& p(n)=\eta p(n-1)+(1-\eta)\left[y_{1}(n)-y_{2}(n)\right]^{2} \\
& \alpha(n)=\alpha(n-1)+\frac{\mu_{\alpha}}{p(n)} e_{d}(n)\left[y_{1}(n)-y_{2}(n)\right] \varphi^{\prime}[\alpha(n-1)]
\end{aligned}
$$

Fim

Fonte: Autora.

\subsubsection{Resultados de simulações}

Os resultados desta seção foram divididos em duas partes. Na primeira, foram utilizados canais de comunicação comumente utilizados em simulações de equalização. Na segunda parte, os canais foram gerados a partir de funções de espalhamento de ponto (PSFs) utilizadas para gerar as imagens degradadas nas simulações do Capítulo 4.

\subsubsection{Canais não baseados em PSF}

Para verificar o comportamento da combinação do equalizador CMA com o equalizador NLMS-DD nos esquemas de minimização mostrados nesta seção, foram realizadas simulações considerando um sistema de comunicação como o esquematizado na Figura 9, em que o sinal transmitido $a(n)$ é um sinal 8-PAM, assumido independente e identicamente distribuído (iid) e o canal de comunicação é modelado por um filtro FIR sem ruído, considerando ao todo $10^{5}$ iterações. A função de transferência do filtro é inicialmente $H_{1}(z)=0,2+z^{-1}+0,2 z^{-2}$, sendo alterada para $H_{2}(z)=0,3+z^{-1}+0,3 z^{-2}$ na metade das iterações para representar uma variação abrupta no canal. Como comparação, utiliza-se a equalização com o NLMS supervisionado, já que essa configuração garante os melhores resultados devido ao uso da sequência de treinamento. Para avaliar o desempenho dos algoritmos nas simulações deste capítulo, utilizou-se o erro quadrático médio $\mathrm{E}\left\{e^{2}(n)\right\}$, denotado aqui simplesmente como MSE (mean square error), em que $e(n)=a(n-\Delta)-y(n)$, isto é, a diferença entre o sinal 
transmitido atrasado de $\Delta$ amostras e a saída do equalizador.

Na Figura 12, são mostrados em duas colunas os resultados da combinação para os esquemas de minimização discutidos. Essas colunas mostram os valores das saídas $y_{1}(n), y_{2}(n)$, $y(n)$ e $y_{\mathrm{NLMS}}(n)$, os MSEs da combinação e de cada equalizador, e o valor médio do parâmetro de mistura $\mathrm{E}\{\lambda(n)\}$, calculados para uma média de 100 realizações. As curvas de MSE ainda foram suavizadas por um filtro de média móvel de 512 coeficientes para facilitar a visualização das suas características.

A coluna da esquerda trata da combinação convexa com erro do CMA $\varepsilon(n)$, cujos parâmetros utilizados são: $\mu_{\alpha}=5,0 \times 10^{-4}, \mu_{1}=10^{-6}, \mu_{2}=\mu_{\mathrm{NLMS}}=10^{-2}$ e $\alpha^{+}=4$. E a coluna da direita refere-se à combinação convexa com o erro de decisão global $e_{d}(n)$, com $\mu_{\alpha}=10^{-1}, \mu_{1}=10^{-6}, \mu_{2}=\mu_{\mathrm{NLMS}}=10^{-2}$ e $\alpha^{+}=4$. Sendo os números de coeficientes dos filtros iguais a $M_{1}=M_{2}=M_{\mathrm{NLMS}}=11$ para todos os casos. Em geral, os dois tipos de combinação simulados apresentaram resultados muito próximos, assim a análise feita a seguir é considerada para ambos os casos.

Analisando as iterações até $n=5,0 \times 10^{4}$, onde canal é $H_{1}(z)=0,2+z^{-1}+0,2 z^{-2}$, verifica-se que as saídas $y_{1}(n)$ convergem no início, embora suas estimativas não sejam boas, já as saídas $y_{2}(n)$ partem de estimativas ruins e convergem rapidamente para estimativas melhores que as de $y_{1}(n)$. Ao observar as saídas $y(n)$ juntamente com os valores de MSE e $\mathrm{E}\{\lambda(n)\}$, nota-se que inicialmente $\mathrm{E}\{\lambda(n)\} \approx 1$, o MSE da combinação se aproxima do MSE do CMA e as estimativas de $y(n)$ são ruins. Após aproximadamente $n=10^{4}$ iterações, $\mathrm{E}\{\lambda(n)\} \approx 0$, o MSE da combinação se aproxima do MSE do NLMS-DD e as estimativas de $y(n)$ melhoram. Isso indica que no início das iterações o desempenho do CMA é superior ao do NLMS-DD, que precisa do CMA para convergir, e por isso $\mathrm{E}\{\lambda(n)\} \approx 1$. Após $n=10^{4}$, o NLMS-DD já convergiu para um desempenho melhor que o do CMA, portanto $\mathrm{E}\{\lambda(n)\} \approx 0$. Quando o canal passa a ser $H_{2}(z)=0,3+z^{-1}+0,3 z^{-2}$, após a iteração $n=5,0 \times 10^{4}$, as estimativas dos filtros pioram por conta do reajuste dos coeficientes. Como o CMA converge primeiro, a combinação volta a ser dominada pelo CMA. Assim, $\operatorname{E}\{\lambda(n)\} \approx 1$, tornando o MSE da combinação próximo ao do CMA e $y(n) \approx y_{1}(n)$. Conforme as decisões melhoram, a combinação passa a ser dominada novamente pelo NLMS-DD, e portanto $\mathrm{E}\{\lambda(n)\} \approx 0$, tornando o MSE da combinação próximo ao do NLMS-DD e $y(n) \approx y_{2}(n)$. 
Figura 12 - Exemplo de combinação convexa de um equalizador CMA com um NLMS-DD, e um equalizador NLMS supervisionado para um sinal 8-PAM. Na coluna da esquerda, usa-se $\varepsilon(n)$ com $\mu_{\alpha}=5,0 \times 10^{-4}, \mu_{1}=10^{-6}, \mu_{2}=\mu_{\mathrm{NLMS}}=10^{-2}$ e $\alpha^{+}=4$. E na coluna da direita usa-se $e_{d}(n)$, com $\mu_{\alpha}=10^{-1}, \mu_{1}=10^{-6}, \mu_{2}=\mu_{\mathrm{NLMS}}=10^{-2}$ e $\alpha^{+}=4$. Sendo os números de coeficientes dos filtros iguais a $M_{1}=M_{2}=M_{\mathrm{NLMS}}=11$ para todos os casos e o MSE e $\mathrm{E}\{\lambda(n)\}$ calculados para uma média de 100 realizações.
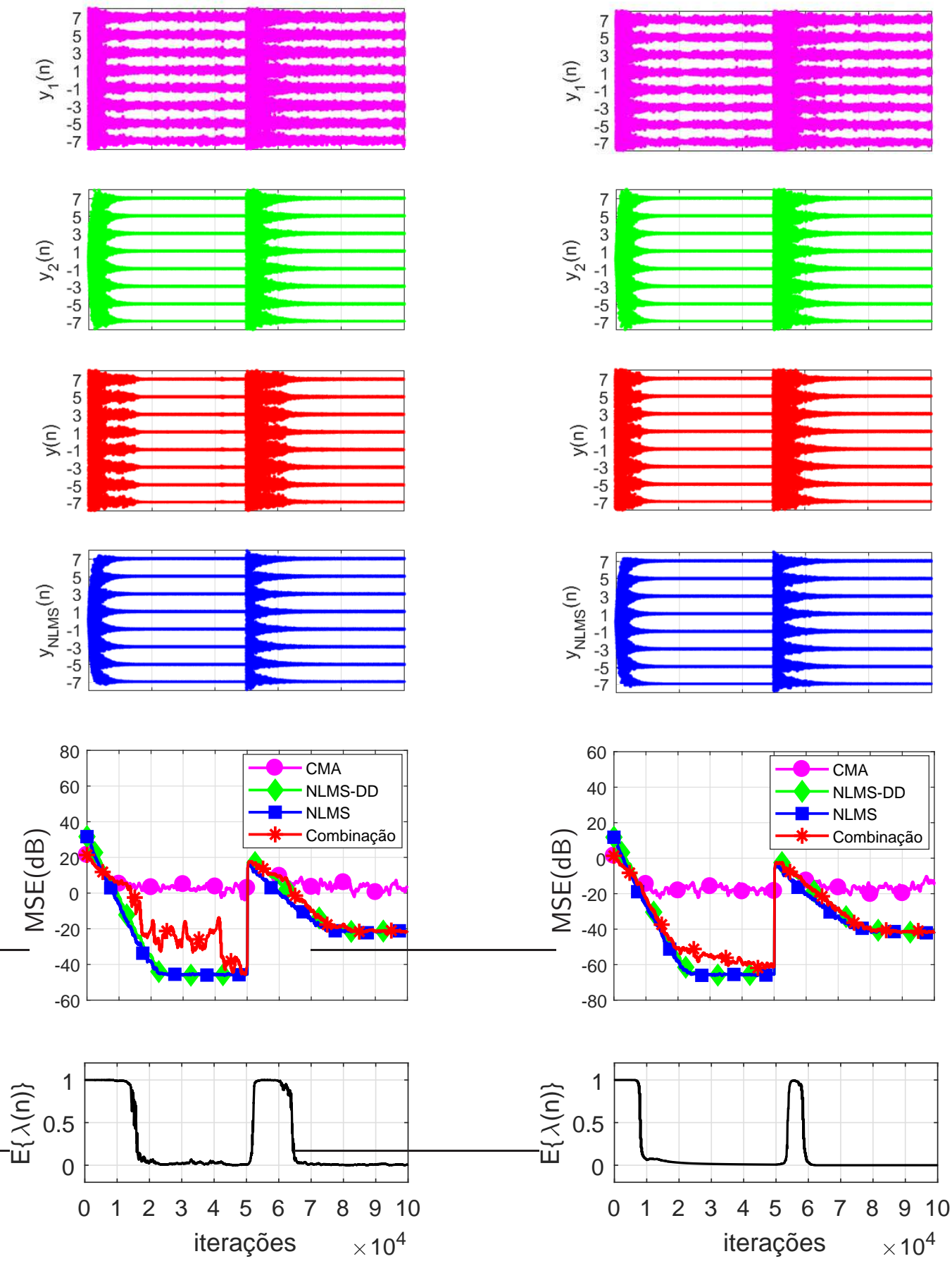

Fonte: Autora. 
Dessa forma, constata-se que o NLMS-DD só converge após o CMA convergir. Isso significa que possivelmente quando houver mudanças no canal, o CMA dominará a combinação até o desempenho do NLMS-DD melhorar. Portanto, o bom funcionamento do NLMS-DD depende do desempenho do CMA. Comparando os resultados da combinação com os do equalizador NLMS supervisionado, observa-se que tanto a saída $y(n)$ quanto o MSE da combinação possuem comportamento muito próximo ao do NLMS supervisionado, o que torna o desempenho desse esquema, apesar de cego, comparável ao de uma solução supervisionada.

Como observado, os dois tipos de combinação simulados apresentaram comportamentos semelhantes para o MSE e para $\mathrm{E}\{\lambda(n)\}$. Assim como em (SILVA; ARENAS-GARCÍA, 2013), o passo de adaptação $\mu_{\alpha}$ foi maior para a combinação com o erro de decisão $e_{d}(n)$ do que para a combinação com o erro do $\mathrm{CMA} \varepsilon(n)$, porém $\mathrm{E}\{\lambda(n)\}$ não apresentou o mesmo comportamento oscilatório que foi verificado naquele trabalho. Uma possível explicação, é o fato dos sinais utilizados aqui serem apenas do tipo PAM, e portanto seus símbolos estão dispostos na reta real.

Na Figura 13 são mostradas as curvas de taxa de erro de símbolo (SER) em regime em função da razão sinal-ruído (SNR) para o CMA, NLMS supervisionado e a combinação convexa com erro de decisão global, considerando o canal $H_{2}(z)=0,3+z^{-1}+0,3 z^{-2}$. As taxas de erro foram calculadas após a convergência dos algoritmos comparando a sequência transmitida com a obtida na saída do decisor e contando o número de erros. Foram desprezados $10^{5}$ símbolos devido à convergência e usados $10^{6}$ símbolos para estimar a SER para cada valor de SNR. Observa-se que até SNR $=20,0 \mathrm{~dB}$ a SER do CMA e a do NLMS supervisionado são iguais, enquanto a da combinação é um pouco maior. A partir de $\mathrm{SNR}=22,5 \mathrm{~dB}$, a SER da combinação passa a ser igual a do NLMS supervisionado e a do CMA torna-se maior que ambas, comprovando o bom desempenho da combinação. 
Figura 13 - SER em regime em função da SNR para o CMA, NLMS supervisionado e a combinação do CMA com o NLMS-DD para o canal $H_{2}(z)=0,3+z^{-1}+0,3 z^{-2}$.

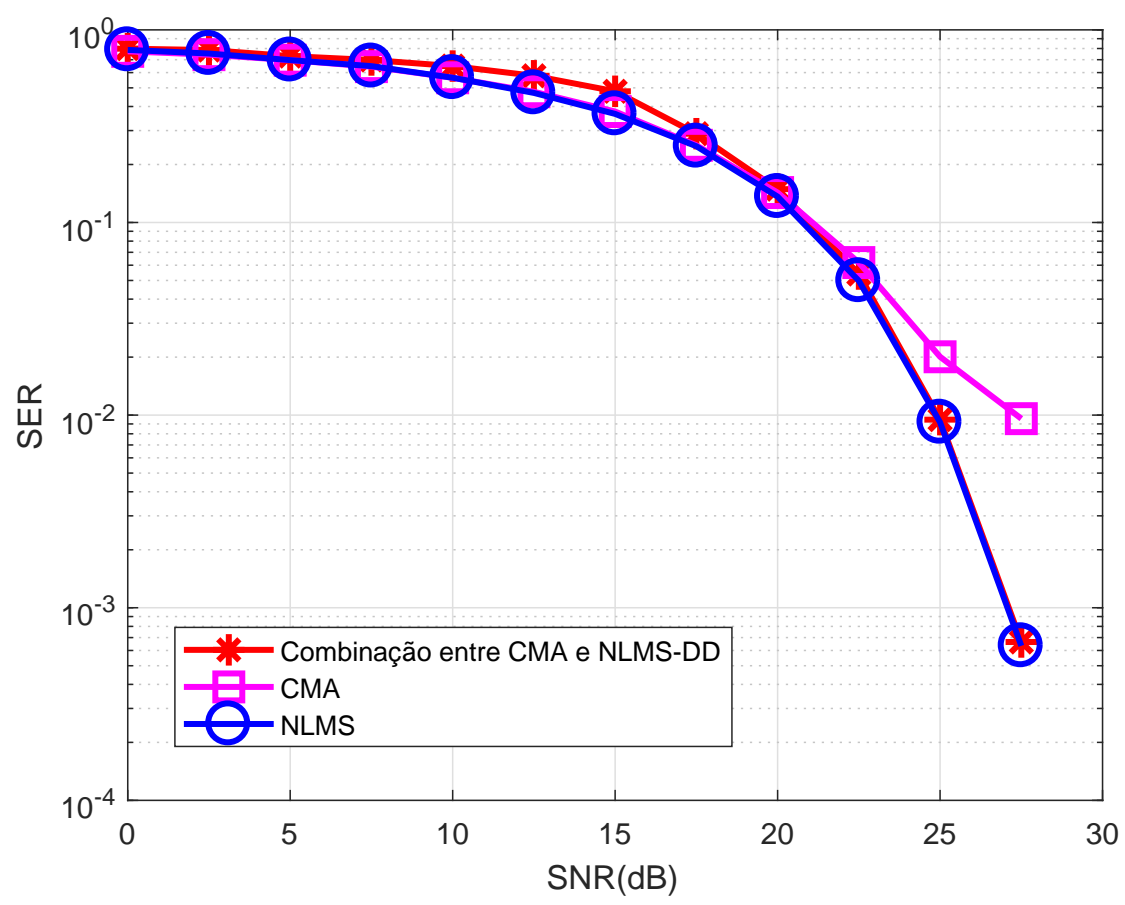

Fonte: Autora.

\subsubsection{Canais baseados em PSF}

Com o objetivo de entender melhor a dificuldade de restaurar imagens distorcidas por PSFs comumente utilizadas na literatura, consideram-se a seguir algumas simulações em que os canais de comunicação foram gerados a partir de PSFs. Dentre elas, o blur gaussiano, amplamente utilizado em programas de edição de imagens (SHAPIRO; STOCKMAN, 2001), e o blur de desfocagem uniforme, proveniente do desajuste do foco de uma câmera (BERTERO; BOCCACCI, 1998). Ambos podem ser gerados pela função fspecial do MATLAB. As PSFs usadas são representadas por matrizes simétricas de dimensão $L \times L$ e suas características são levadas em conta na obtenção da resposta ao pulso unitário "equivalente" para um canal de comunicação. Assim, define-se uma função $g(\cdot)$, que gera uma resposta ao pulso unitário $h(n)$ a partir de uma PSF $\mathbf{H}\left(k_{1}, k_{2}\right)$, descrita no exemplo a seguir. 
Considerando $\mathbf{H}\left(k_{1}, k_{2}\right)$ uma matriz $5 \times 5$, dada por

$$
\mathbf{H}\left(k_{1}, k_{2}\right)=\left(\begin{array}{ccccc}
0 & 0,0170 & 0,381 & 0,0170 & 0 \\
0,0170 & 0,0784 & 0,0796 & 0,0784 & 0,0170 \\
0,381 & 0,0796 & 0,0796 & 0,0796 & 0,381 \\
0,0170 & 0,0784 & 0,0796 & 0,0784 & 0,0170 \\
0 & 0,0170 & 0,381 & 0,0170 & 0
\end{array}\right),
$$

e obtida por meio da função fspecial('disk', 2) do MATLAB, nota-se que a matriz é simétrica, as linhas 1 e 5 e as linhas 2 e 4 são idênticas, assim como as colunas 1 e 5 e as colunas 2 e 4 . Essas características garantem uma espécie de simetria circular e por isso a resposta ao pulso unitário correspondente é considerada simétrica também. Os coeficientes de $h(n)$ podem ser obtidos percorrendo a matriz $\mathbf{H}\left(k_{1}, k_{2}\right)$ nas direções mostradas nos esquemas das Figuras $14 \mathrm{e}$ 15 , em que o elemento central da matriz coincide com o elemento central de $h(n)$. Portanto, a função $g(\cdot)$ pode seguir as sequências indicadas nos esquemas das Figuras 14 e 15 selecionando os elementos destacados em $\mathbf{H}\left(k_{1}, k_{2}\right)$.

Figura 14 - Exemplo com uma das sequências possíveis para a função $g(\cdot)$ percorrer uma determinada PSF e gerar uma resposta ao pulso unitário $h(n)$.

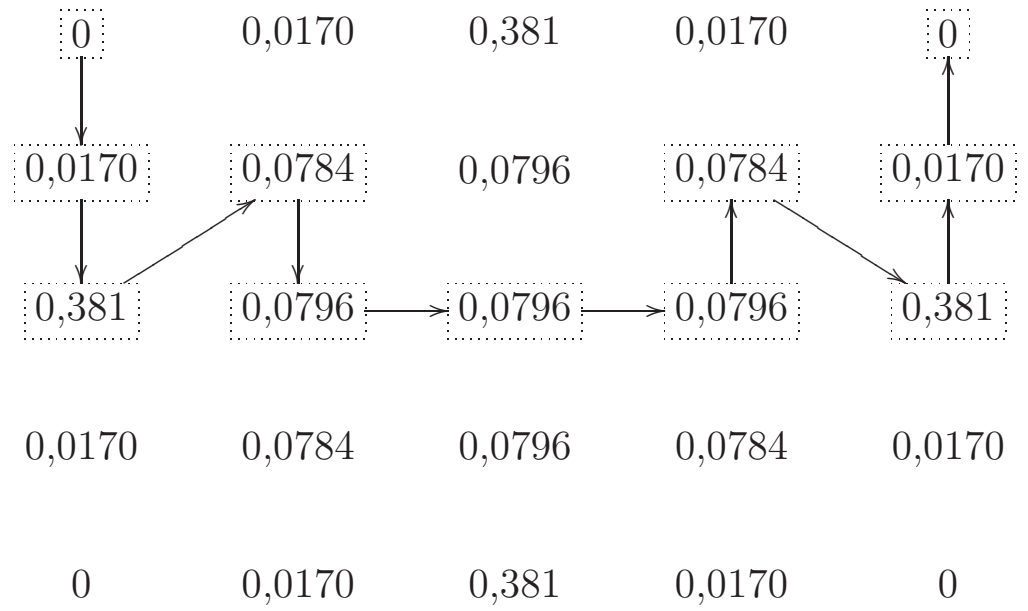

Fonte: Autora. 
Figura 15 - Exemplo com uma das sequências possíveis para a função $g(\cdot)$ percorrer uma determinada PSF e gerar uma resposta ao pulso unitário $h(n)$.

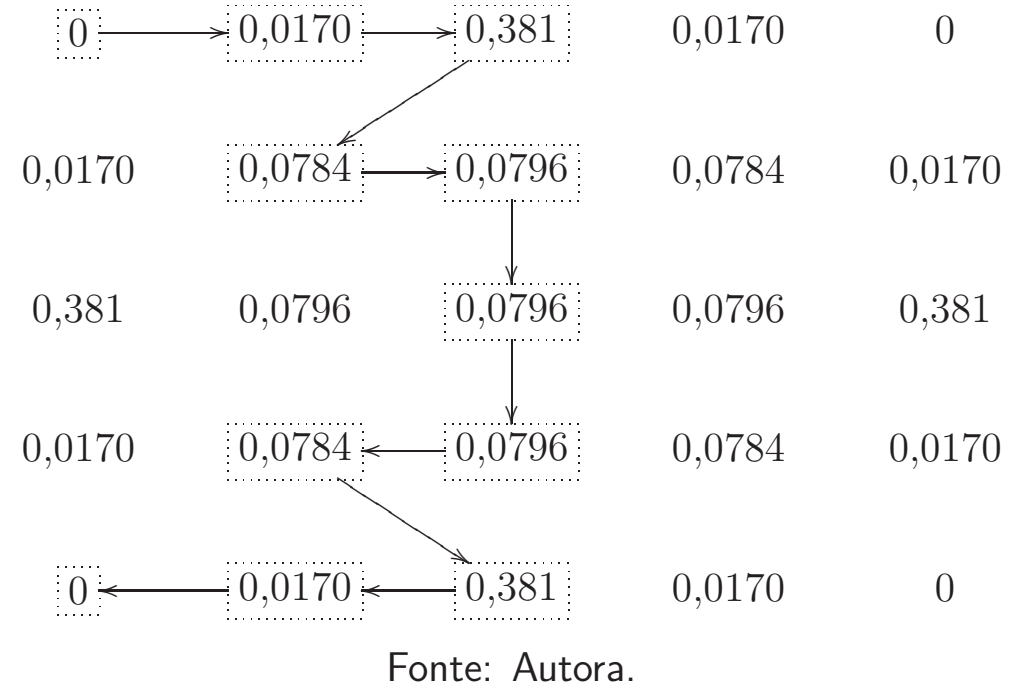

Como os coeficientes de uma resposta ao pulso unitário FIR $h(n)$ são os mesmos da sua função de transferência $H(z), g(\cdot)$ também pode ser definida como um função que gera a função de transferência $H(z)$ a partir da $\operatorname{PSF} \mathbf{H}\left(k_{1}, k_{2}\right)$, isto é

$$
H(z)=g\left(\mathbf{H}\left(k_{1}, k_{2}\right)\right)
$$

Assim, para o exemplo da Equação (3.15), a função de transferência é

$$
\begin{aligned}
H(z)= & 0,017+0,381 z^{-1}+0,0784 z^{-2} \\
& +0,0796 z^{-3}+0,0796 z^{-4}+0,0796 z^{-5} \\
& +0,0784 z^{-6}+0,381 z^{-7}+0,017 z^{-8} .
\end{aligned}
$$

$\mathrm{Na}$ Tabela 4, são descritos os cenários utilizados nas simulações em que os canais de comunicação são baseados em PSFs e o sinal transmitido é uma sequência assumida iid. Nas simulações, as funções de transferência dos canais foram normalizadas para que não houvesse ganho de potência no canal, facilitando a escolha dos passos de adaptação dos algoritmos. No Capítulo 4, essas PSFs são utilizadas nas simulações para restauração cega de imagens, de maneira que as simulações desta seção visam relacionar o comportamento da equalização com o da restauração. Assim, os sinais de comunicação dos cenários da Tabela 4 possuem o mesmo número de símbolos dos sinais de imagens simulados no Capítulo 4. 
Para esses cenários as simulações foram feitas considerando a combinação com o erro de decisão global $e_{d}(n)$ para $N$ iterações. Para cada cenário, mostra-se na mesma figura as saídas $y_{1}(n), y_{2}(n), y(n)$ e $y_{\mathrm{NLMS}}(n)$, os MSEs de cada algoritmo e o valor médio do parâmetro de mistura $\mathrm{E}\{\lambda(n)\}$ para uma média de 100 realizações, sendo o MSE filtrado por um filtro de média móvel de 512 coeficientes. Em uma segunda figura são mostrados os gráficos que indicam a ocorrência de erro entre o sinal transmitido com atraso $a(n-\Delta)$ e o sinal de saída decidido $\hat{a}(n-\Delta)$, para o CMA, a combinação e o NLMS supervisionado. Nesses gráficos, as iterações que apresentam erros recebem valor 1 e ao contrário 0 , de maneira que a existência de erros é simbolizada por raias de amplitude 1 .

A Figura 16 mostra os resultados para o Cenário 1-A com $N=8,0 \times 10^{4}$ iterações, em que são utilizados $\mu_{\alpha}=10^{-1}, \mu_{1}=2,0 \times 10^{-7}, \mu_{2}=\mu_{\mathrm{NLMS}}=0,5, \alpha^{+}=4$, e os números de coeficientes dos filtros são $M_{1}=M_{2}=M_{\mathrm{NLMS}}=17$. Nota-se que inicialmente $\operatorname{E}\{\lambda(n)\} \approx 1$ e o MSE da combinação é muito próximo ao do NLMS-DD. Ao longo das iterações, o MSE do CMA converge para o patamar de $-2,25 \mathrm{~dB}$ e o do NLMS-DD para o patamar de $-48,0 \mathrm{~dB}$, o que faz $\mathrm{E}\{\lambda(n)\} \approx 0$ e a combinação funciona como o NLMS-DD. Dessa forma, a combinação consegue atingir o mesmo nível de MSE do NLMS supervisionado. Já a Figura 17, com a ocorrência de erro entre o sinal transmitido $a(n-\Delta)$ e a saída decidida $\hat{a}(n-\Delta)$, mostra que os erros no CMA são muito frequentes ao longo das iterações, diferentemente da combinação e do NLMS, cujos erros ocorrem com mais frequência até determinadas iterações e tornam-se nulos depois. Essas iterações correspondem àquelas em que os MSEs desses algoritmos entram em convergência e diminuem, conforme mostrado na Figura 16. 
Tabela 4: Cenários de simulações nas quais os canais de comunicação são gerados a partir de PSFs.

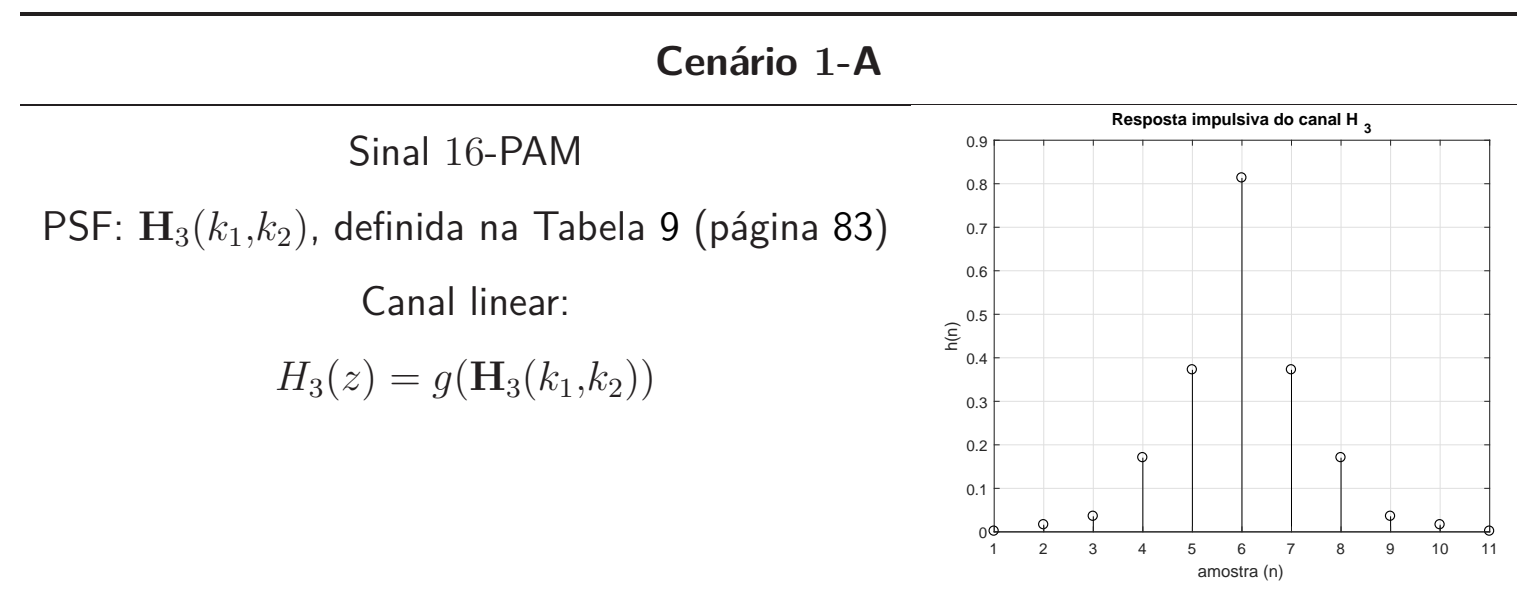

\section{Cenário 1-B}

Sinal 32-PAM

PSF: $\mathbf{H}_{4}\left(k_{1}, k_{2}\right)$, definida na Tabela 9 (página 83)

Canal linear:

$$
H_{4}(z)=g\left(\mathbf{H}_{4}\left(k_{1}, k_{2}\right)\right)
$$

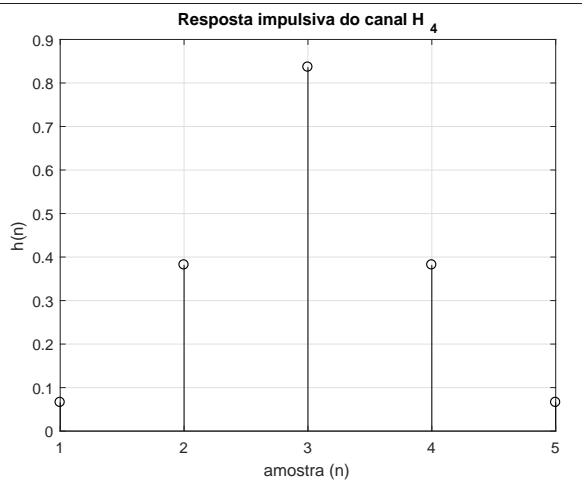

\section{Cenário 1-C}

\section{Sinal 32-PAM}

PSF: $\mathbf{H}_{5}\left(k_{1}, k_{2}\right)$, definida na Tabela 9 (página 83)

Canal linear:

$$
H_{5}(z)=g\left(\mathbf{H}_{5}\left(k_{1}, k_{2}\right)\right)
$$

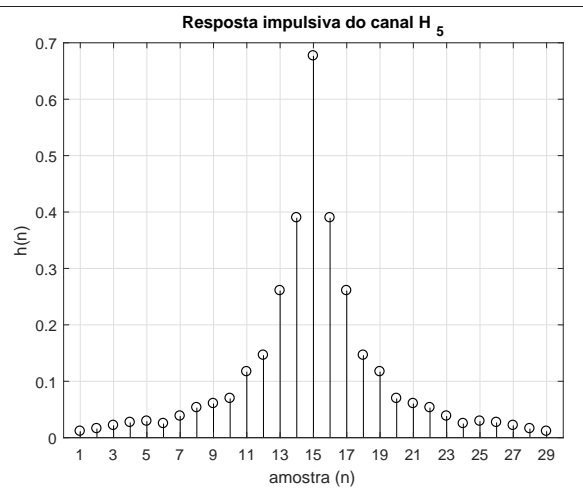

Fonte: Autora. 
Figura 16 - Exemplo de combinação convexa de um equalizador CMA com um NLMS-DD, e de um equalizador NLMS supervisionado para o Cenário 1-A da Tabela 4 (página 37). Em que se usa o erro de decisão global $e_{d}(n)$, com $\mu_{\alpha}=10^{-1}, \mu_{1}=2,0 \times 10^{-7}, \mu_{2}=\mu_{\mathrm{NLMS}}=0,5, \alpha^{+}=4$, e os números de coeficientes dos filtros são $M_{1}=M_{2}=M_{\mathrm{NLMS}}=17$. O MSE e $\mathrm{E}\{\lambda(n)\}$ são calculados para 100 realizações.
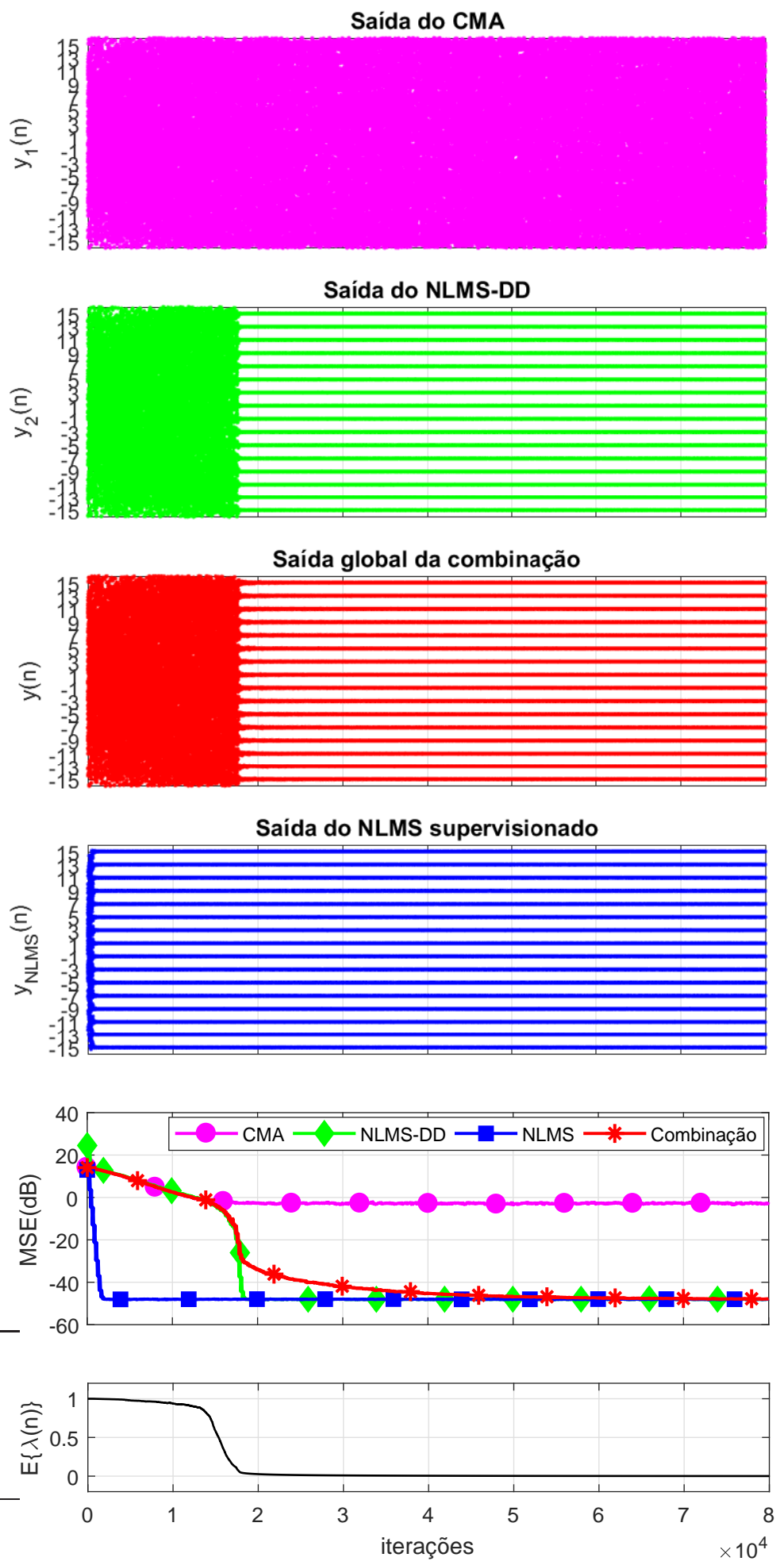

Fonte: Autora. 
Figura 17 - Diferença entre o sinal transmitido com atraso $a(n-\Delta)$ e o sinal decidido $\hat{a}(n-\Delta)$, para a combinação do CMA com o NLMS-DD com o erro de decisão global $e_{d}(n)$, dado o Cenário 1-A da Tabela 4 (página 37).

CMA: Taxa de Erro $=2,2 \times 10^{-1}$

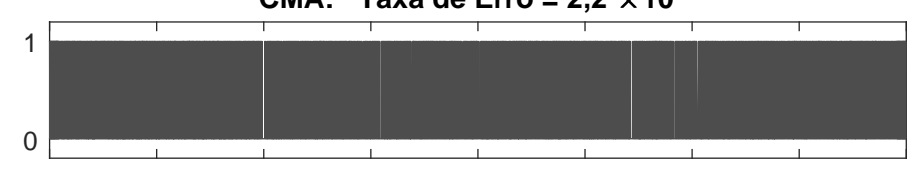

Combinação CMA $\times$ NLMS-DD: Taxa de Erro = 1,1 $\times 10^{-1}$

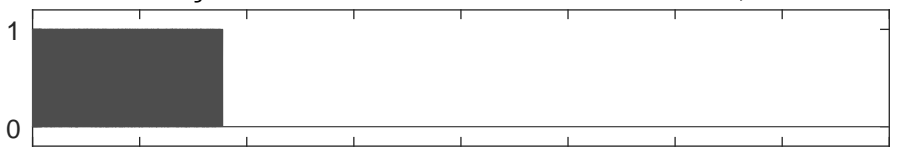

NLMS supervisionado: Taxa de Erro $=3,3 \times 10^{-3}$

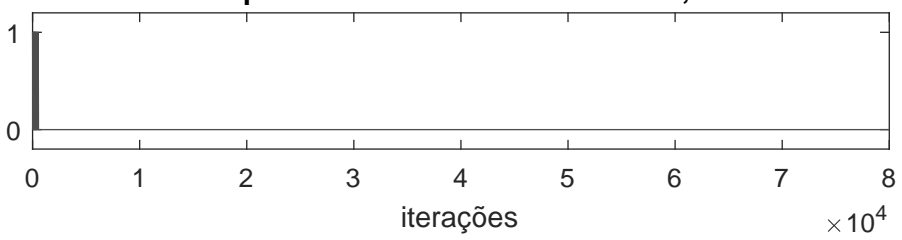

Fonte: Autora.

A Figura 18 mostra os resultados para o Cenário 1-B considerando $N=8,0 \times 10^{4}$ iterações, em que são utilizados $\mu_{\alpha}=10^{-1}, \mu_{1}=2,0 \times 10^{-8}, \mu_{2}=\mu_{\mathrm{NLMS}}=10^{-1}, \alpha^{+}=4$, e os números de coeficientes dos filtros são $M_{1}=M_{2}=M_{\mathrm{NLMS}}=9$. No início das iterações, $\mathrm{E}\{\lambda(n)\} \approx 1$ e o MSE da combinação é próximo ao do CMA. Ao longo das iterações, o MSE do CMA converge para o patamar de $2,32 \mathrm{~dB}$ e o do NLMS-DD converge para $-27,0 \mathrm{~dB}$. Após aproximadamente a iteração $n=3,2 \times 10^{4}, \mathrm{E}\{\lambda(n)\} \approx 0$ e a combinação chaveia para o modo DD, atingindo o mesmo nível de MSE que o NLMS supervisionado. A Figura 19, mostra que a ocorrência de erro entre o sinal transmitido $a(n-\Delta)$ e a saída decidida $\hat{a}(n-\Delta)$ é frequente ao longo das iterações para o CMA, enquanto para a combinação e para o NLMS esses erros são frequentes até o início da convergência e tornam-se nulos nas demais iterações. 
Figura 18 - Exemplo de combinação convexa de um equalizador CMA com um NLMS-DD, e de um equalizador NLMS supervisionado para o Cenário 1-B da Tabela 4 (página 37). Em que se usa o erro de decisão global $e_{d}(n)$, com $\mu_{\alpha}=10^{-1}, \mu_{1}=2,0 \times 10^{-8}, \mu_{2}=\mu_{\mathrm{NLMS}}=10^{-1}, \alpha^{+}=4$, e os números de coeficientes dos filtros iguais a $M_{1}=M_{2}=M_{\mathrm{NLMS}}=9$. O MSE e $\mathrm{E}\{\lambda(n)\}$ são calculados para 100 realizações.
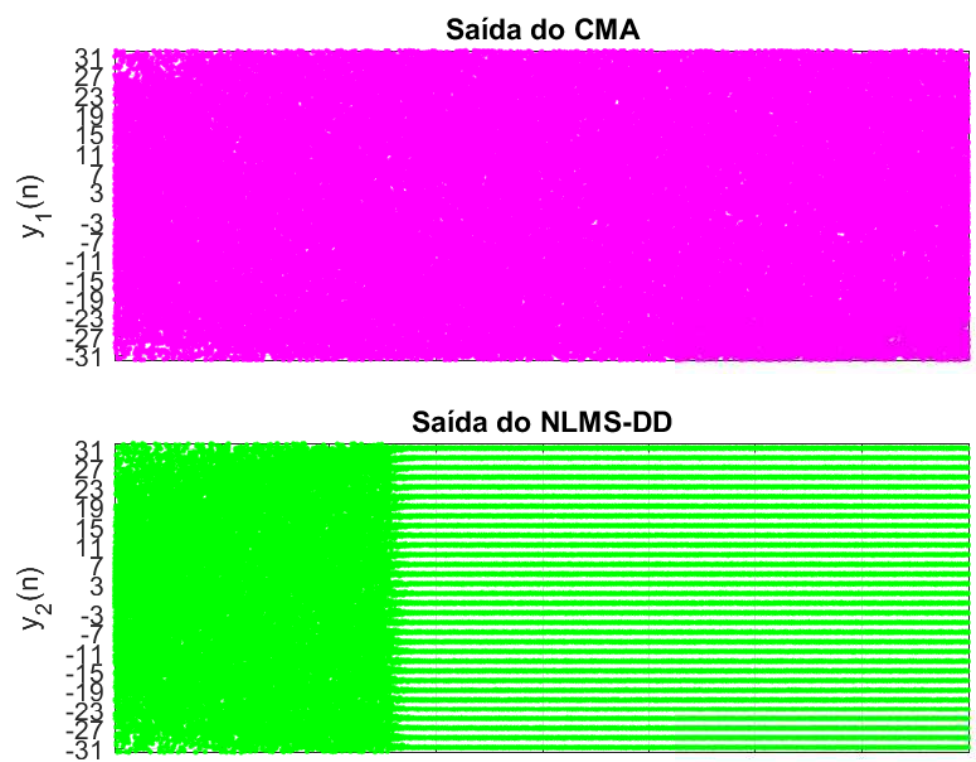

Saída global da combinação

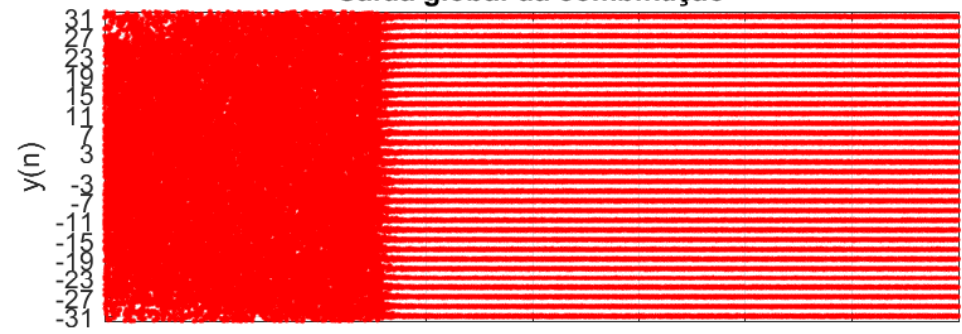

Saída do NLMS supervisionado
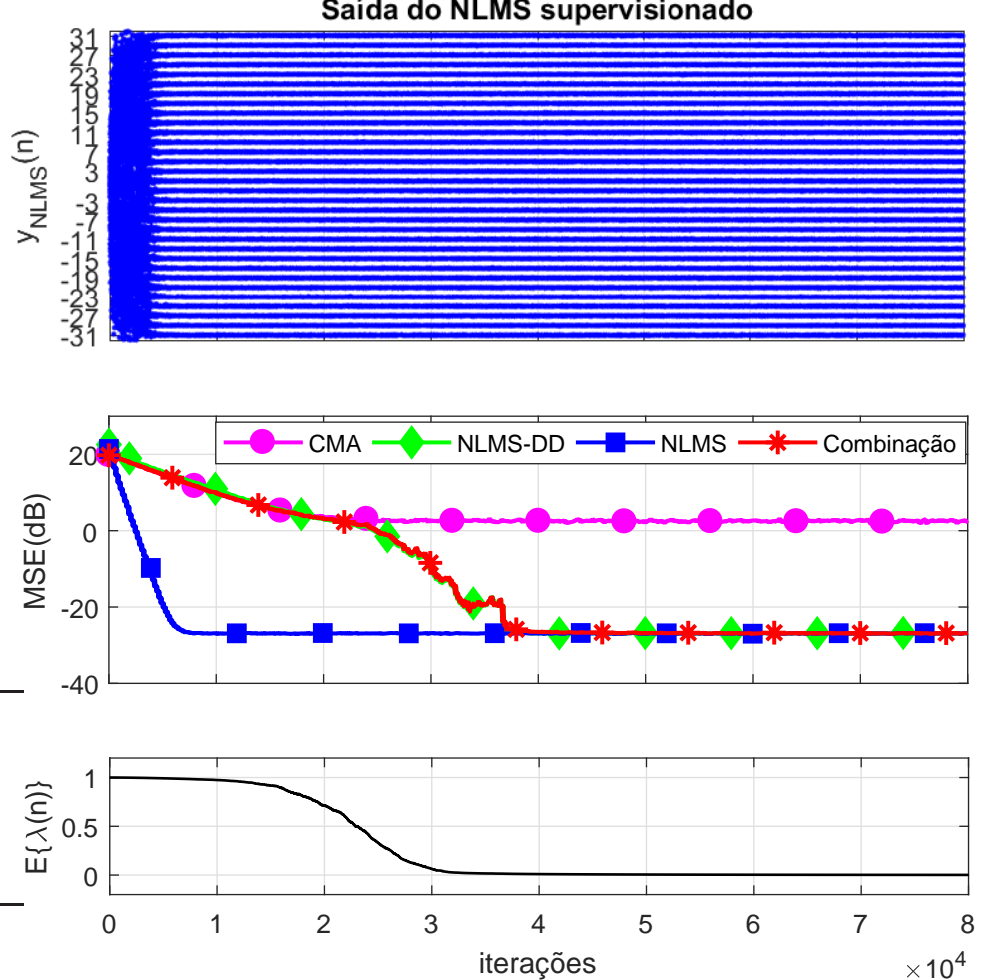

Fonte: Autora. 
Figura 19 - Diferença entre o sinal transmitido com atraso $a(n-\Delta)$ e o sinal decidido $\hat{a}(n-\Delta)$, para a combinação do CMA com o NLMS-DD com o erro de decisão global $e_{d}(n)$, dado o Cenário 1-B da Tabela 4 (página 37).

CMA: Taxa de Erro $=5,0 \times 10^{-1}$

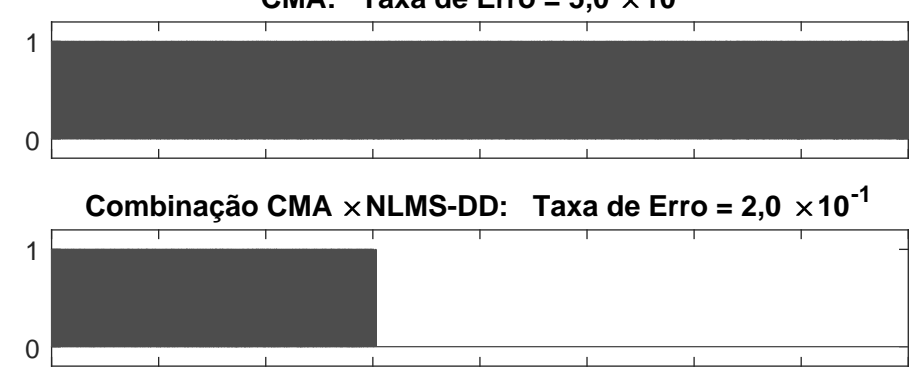

NLMS supervisionado: Taxa de Erro $=2,4 \times 10^{-2}$

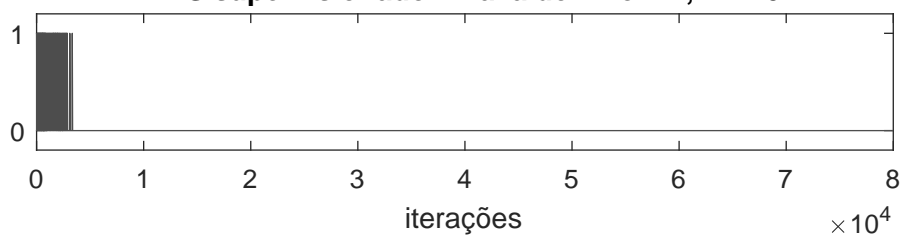

Fonte: Autora.

A Figura 20 mostra os resultados para o Cenário 1-C para $N=10^{6}$ iterações, em que são utilizados $\mu_{\alpha}=10^{-2}, \mu_{1}=2,0 \times 10^{-9}, \mu_{2}=\mu_{\mathrm{NLMS}}=0,5, \alpha^{+}=4$, e os números de coeficientes dos filtros são iguais a $M_{1}=49$ e $M_{2}=M_{\mathrm{NLMS}}=47$. Inicialmente, quando $\mathrm{E}\{\lambda(n)\} \approx 1$, o MSE da combinação é igual ao do CMA. Após aproximadamente a metade das iterações, o MSE do CMA convergiu para $-4,68 \mathrm{~dB}$ e o do NLMS-DD converge para $-27,7 \mathrm{~dB}$, fazendo $\mathrm{E}\{\lambda(n)\} \approx 0$ e a combinação chaveia para o modo DD. Dessa forma, a combinação atinge o mesmo nível de MSE do NLMS supervisionado. A Figura 21 mostra que a ocorrência de erro entre o sinal transmitido $a(n-\Delta)$ e a saída decidida $\hat{a}(n-\Delta)$ de cada algoritmo, mais uma vez, é mais frequente para o CMA. Novamente, para a combinação e o NLMS, esses erros são frequentes até o início da convergência, tornando-se inexistentes em seguida. 
Figura 20 - Exemplo de combinação convexa de um equalizador CMA com um NLMS-DD, e de um equalizador NLMS supervisionado para o Cenário 1-C da Tabela 4 (página 37). Em que se usa o erro de decisão global $e_{d}(n)$, com $\mu_{\alpha}=10^{-2}, \mu_{1}=2,0 \times 10^{-9}, \mu_{2}=\mu_{\mathrm{NLMS}}=0,5, \alpha^{+}=4$, e os números de coeficientes dos filtros são iguais a $M_{1}=49$ e $M_{2}=M_{\mathrm{NLMS}}=47$. O MSE e E $\{\lambda(n)\}$ são calculados para 100 realizações.
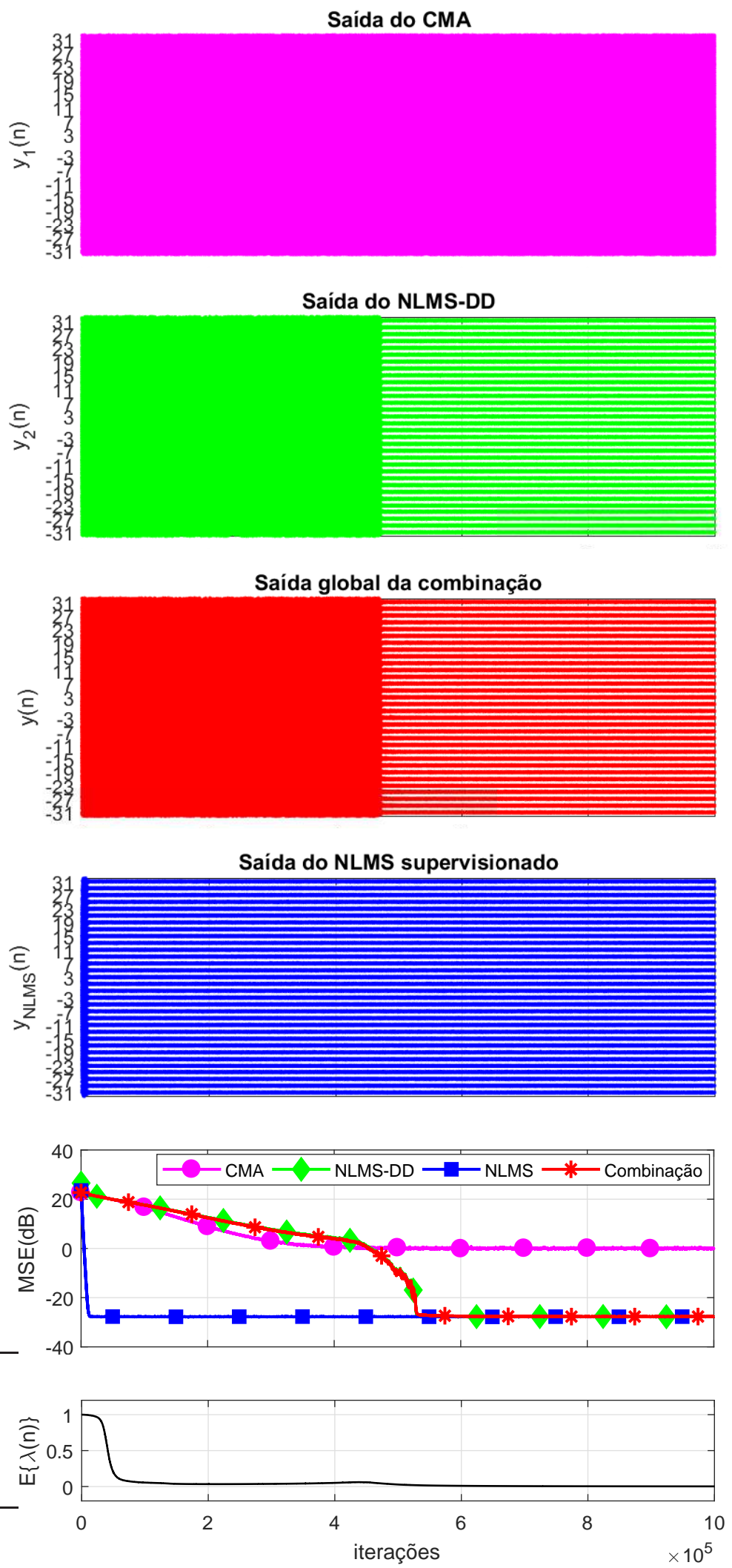

Fonte: Autora. 
Figura 21 - Diferença entre o sinal transmitido com atraso $a(n-\Delta)$ e o sinal decidido $\hat{a}(n-\Delta)$, para a combinação do CMA com o NLMS-DD com o erro de decisão global $e_{d}(n)$, dado o Cenário 1-C da Tabela 4 (página 37).

CMA: Taxa de Erro $=4,4 \times 10^{-1}$

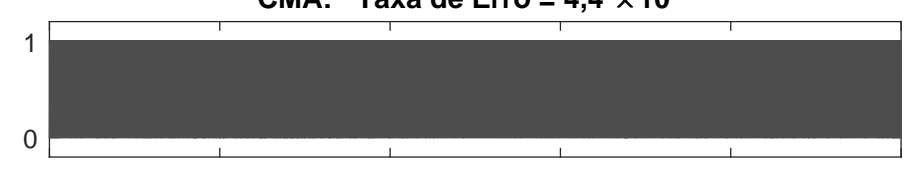

Combinação CMA $\times$ NLMS-DD: Taxa de Erro $=3,4 \times 10^{-1}$

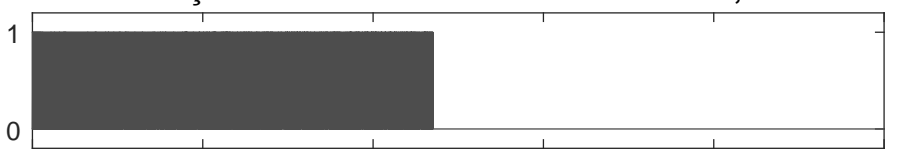

NLMS supervisionado: Taxa de Erro $=3,3 \times 10^{-3}$

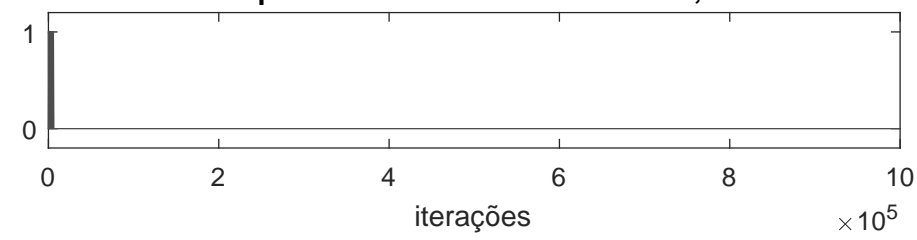

Fonte: Autora.

\subsection{Combinação convexa baseada em soluções não lineares para equalização}

Considerando as vantagens do DFE e do KAF para canais com nulos espectrais e/ou não lineares, é possível tirar proveito dessas soluções para a equalização cega de canais por meio do esquema de combinação convexa discutido na Seção 3.1. Assim, são propostos dois esquemas: a combinação convexa entre o CMA e o DFE no modo de decisão direta adaptado com o NLMS, denominado aqui como DFE-NLMS-DD; e a combinação convexa entre o CMA e o algoritmo QKLMS no modo de decisão direta, o QKLMS-DD. Essas combinações são discutidas a seguir.

\subsubsection{Combinação convexa entre o CMA e o DFE-NLMS-DD}

O esquema da combinação convexa entre o CMA e o DFE-NLMS-DD com o erro de decisão global $e_{d}(n)$ é mostrado na Figura 22, em que a atualização do vetor de coeficientes concatenados é dada por

$$
\mathbf{w}_{f b}(n)=\mathbf{w}_{f b}(n-1)+\frac{\mu_{2}}{\delta+\left\|\mathbf{u}_{f b}(n)\right\|^{2}} e_{d 2}(n) \mathbf{u}_{f b}(n),
$$

sendo $\frac{\mu_{2}}{\delta+\left\|\mathbf{u}_{f b}(n)\right\|^{2}}$ o passo de adaptação normalizado e $e_{d 2}=\hat{a}(n-\Delta)-y_{2}(n)$. 
Figura 22 - Combinação convexa de um equalizador CMA com um DFE-NLMS-DD, com $\lambda(n)$ obtido a partir da minimização de $e_{d}^{2}(n)$.

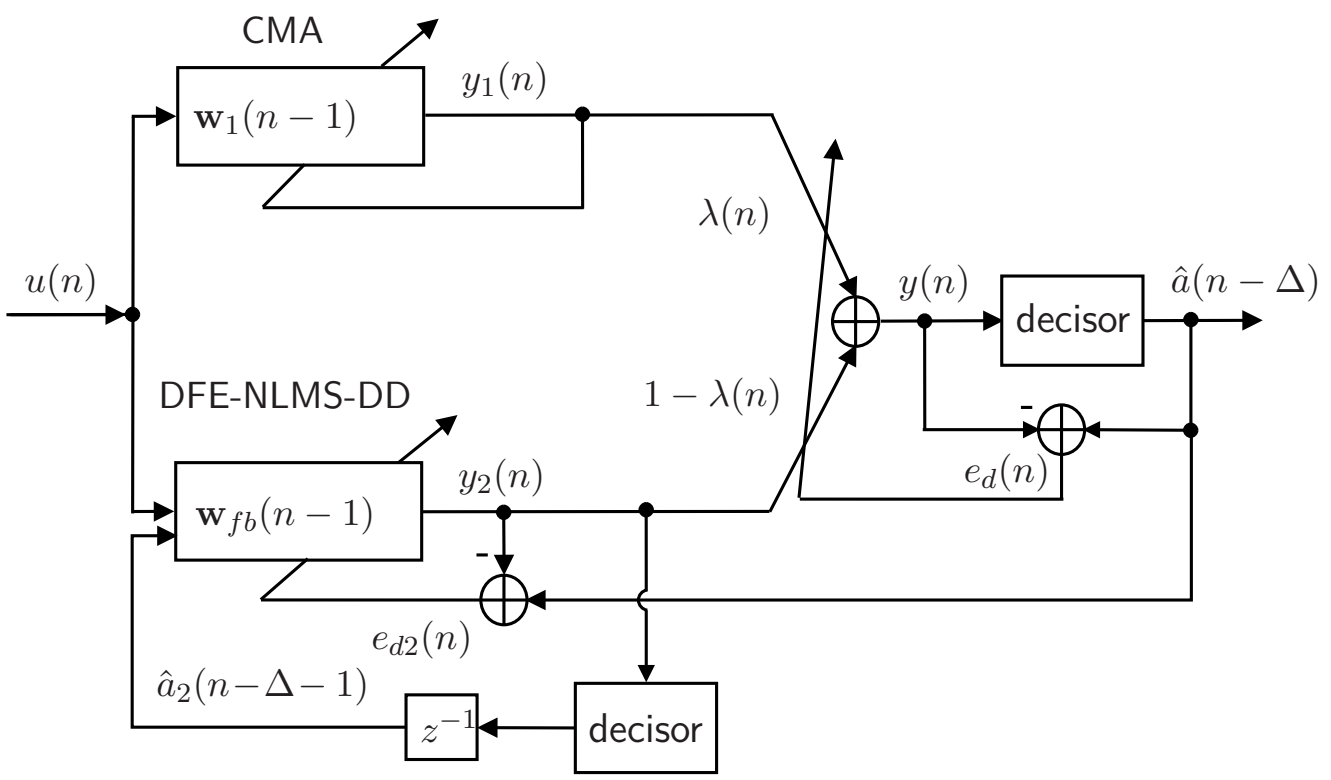

Fonte: Autora.

As equações que implementam o algoritmo de combinação convexa entre o CMA e o DFE-NLMS-DD com o erro de decisão global $e_{d}(n)$ são mostradas na Tabela 5 . Vale ressaltar que o esquema de combinação com o erro do CMA pode ser utilizado substituindo $e_{d}(n)$ na atualização da variável auxiliar $\alpha(n)$ por $\varepsilon(n)$ (definido na Equação (2.8), página 13). 
Tabela 5: Sumário do algoritmo de combinação convexa entre o CMA e o DFE-NLMS-DD, com minimização do erro de decisão global ao quadrado $e_{d}^{2}(n)$.

Combinação convexa entre o CMA e o DFE-NLMS-DD

Escolha dos passos de adaptação $\mu_{1}, \mu_{2}>0 ; \quad r=\frac{\mathrm{E}\left\{a^{4}(n)\right\}}{\mathrm{E}\left\{a^{2}(n)\right\}}$;

Inicialização

$\mathbf{w}_{1}(0)=\left[\begin{array}{lllllll}0 & \cdots & 0 & 1 & 0 & \cdots & 0\end{array}\right]^{T} ; \quad \mathbf{w}_{f b}(0)=\mathbf{0}_{\left(M_{f}+M_{b}\right)}$

$\hat{\mathbf{a}}_{\Delta}(1)=\mathbf{0}_{M_{b}} ; \quad \alpha(0)=\alpha^{+} ; \quad p(0)=1 ; \quad \eta=0,9$

Cálculos

Para $n=1,2, \cdots, N$, faça:

\% Cálculo das saídas:

$\mathbf{u}_{f b}(n)=\left[\begin{array}{ll}\mathbf{u}^{T}(n) & \hat{\mathbf{a}}_{\Delta}^{T}(n)\end{array}\right]^{T}$

$y_{1}(n)=\mathbf{u}^{T}(n) \mathbf{w}_{1}(n-1)$

$y_{2}(n)=\mathbf{u}_{f b}(n)^{T} \mathbf{w}_{f b}(n-1)$

Se $|\alpha(n-1)|>\alpha^{+}: \alpha(n-1) \leftarrow \alpha^{+} \operatorname{sign}[\alpha(n-1)]$

$\lambda(n)=\varphi[\alpha(n-1)]=\frac{\operatorname{sgm}[\alpha(n-1)]-\operatorname{sgm}\left[-\alpha^{+}\right]}{\operatorname{sgm}\left[\alpha^{+}\right]-\operatorname{sgm}\left[-\alpha^{+}\right]}$

$y(n)=\lambda(n) y_{1}(n)+[1-\lambda(n)] y_{2}(n)$

\% Cálculo dos erros:

$\varepsilon_{1}(n)=\left[r-y_{1}^{2}(n)\right] y_{1}(n)$

$\hat{a}(n-\Delta)=\operatorname{decisor}[y(n)]$

$e_{d 2}(n)=\hat{a}(n-\Delta)-y_{2}(n)$

$e_{d}(n)=\hat{a}(n-\Delta)-y(n)$

\% Atualizações: 
Combinação convexa entre o CMA e o DFE-NLMS-DD

$$
\begin{aligned}
& \mathbf{w}_{1}(n)=\mathbf{w}_{1}(n-1)+\mu_{1} \varepsilon_{1}(n) \mathbf{u}(n) \\
& \mathbf{w}_{f b}(n)=\mathbf{w}_{f b}(n-1)+\frac{\mu_{2}}{\delta+\left\|\mathbf{u}_{f b}(n)\right\|^{2}} e_{d 2}(n) \mathbf{u}_{f b}(n) \\
& \varphi^{\prime}[\alpha(n-1)]=\frac{\operatorname{sgm}[\alpha(n-1)]\{1-\operatorname{sgm}[\alpha(n-1)]\}}{\operatorname{sgm}\left[\alpha^{+}\right]-\operatorname{sgm}\left[-\alpha^{+}\right]} \\
& p(n)=\eta p(n-1)+(1-\eta)\left[y_{1}(n)-y_{2}(n)\right]^{2} \\
& \alpha(n)=\alpha(n-1)+\frac{\mu_{\alpha}}{p(n)} e_{d}(n)\left[y_{1}(n)-y_{2}(n)\right] \varphi^{\prime}[\alpha(n-1)] \\
& \hat{a}_{2}(n-\Delta)=\operatorname{decisor}\left[y_{2}(n)\right] \\
& \hat{\mathbf{a}}_{\Delta}(n)=\left[\begin{array}{llll}
\hat{a}_{2}(n-\Delta) & \hat{a}_{2}(n-\Delta-1) & \cdots & \hat{a}_{2}\left(n-\Delta-M_{b}+1\right)
\end{array}\right]^{T}
\end{aligned}
$$

Fim

Fonte: Autora.

\subsubsection{Combinação convexa entre o CMA e o QKLMS-DD}

A combinação convexa entre o algoritmo CMA e QKLMS-DD é proposta para a equalização cega de canais não lineares. A Figura 23 esquematiza a combinação com o erro de decisão global $e_{d}(n)$, lembrando que o erro do CMA $\varepsilon(n)$ também pode ser utilizado na adaptação da variável auxiliar $\alpha(n)$.

Apesar do CMA não ser uma solução apropriada para a equalização não linear, a combinação pode usar suas estimativas para fazer o QKLMS-DD funcionar adequadamente. Como será observado nas simulações da Seção 3.2.3, em alguns canais não lineares, para que o QKLMS-DD comece a funcionar, basta que o CMA obtenha uma primeira estimativa (mesmo que ruim) do sinal transmitido. A Tabela 6 traz as equações que implementam o algoritmo de combinação entre o CMA e o QKLMS-DD utilizando o erro de decisão $e_{d}(n)$. 
Figura 23 - Combinação convexa de um equalizador CMA com um QKLMS-DD, com $\lambda(n)$ obtido a partir da minimização de $e_{d}^{2}(n)$.

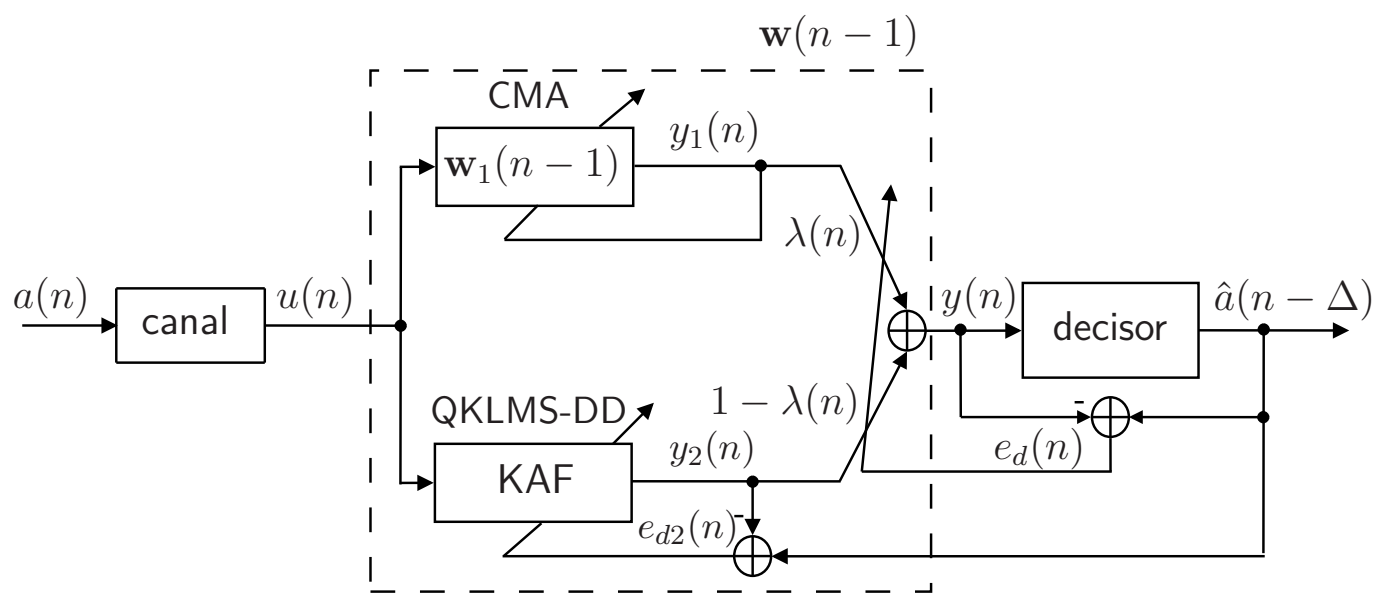

Fonte: Autora.

Tabela 6: Sumário do algoritmo de combinação convexa entre o CMA e o QKLMS-DD, com minimização do erro de decisão global ao quadrado $e_{d}^{2}(n)$.

Combinação convexa entre o CMA e o QKLMS-DD

Escolha dos passos de adaptação $\mu_{1}, \mu_{2}>0$; largura do kernel $\sigma>0$; $\operatorname{limiar} \rho \geq 0 ; r=\frac{\mathrm{E}\left\{a^{4}(n)\right\}}{\mathrm{E}\left\{a^{2}(n)\right\}}$

Inicialização

$\mathbf{w}_{1}(0)=\left[\begin{array}{lllllll}0 & \cdots & 0 & 1 & 0 & \cdots & 0\end{array}\right]^{T} ;$

$\mathbf{b}(1)=[] ; \quad \mathbf{C}(1)=\{\} ;$

$\alpha(0)=\alpha^{+} ; \quad p(0)=1 ; \quad \eta=0,9$

Cálculos

Para $n=1,2, \cdots, N$, faça:

\% Cálculo das saídas:

$y_{1}(n)=\mathbf{u}^{T}(n) \mathbf{w}_{1}(n-1)$

Continuação na próxima página 
Combinação convexa entre o CMA e o QKLMS-DD

$y_{2}(n)=\sum_{i=1}^{N_{c}(n)} b_{i}(n-1) \kappa(\mathbf{u}(n), \mathbf{u}(i))$

Se $|\alpha(n-1)|>\alpha^{+}: \alpha(n-1) \leftarrow \alpha^{+} \operatorname{sign}[\alpha(n-1)]$

$\lambda(n)=\varphi[\alpha(n-1)]=\frac{\operatorname{sgm}[\alpha(n-1)]-\operatorname{sgm}\left[-\alpha^{+}\right]}{\operatorname{sgm}\left[\alpha^{+}\right]-\operatorname{sgm}\left[-\alpha^{+}\right]}$

$y(n)=\lambda(n) y_{1}(n)+[1-\lambda(n)] y_{2}(n)$

\% Cálculo dos erros:

$\varepsilon_{1}(n)=\left[r-y_{1}^{2}(n)\right] y_{1}(n)$

$\hat{a}(n-\Delta)=\operatorname{decisor}[y(n)]$

$e_{d 2}(n)=\hat{a}(n-\Delta)-y_{2}(n)$

$e_{d}(n)=\hat{a}(n-\Delta)-y(n)$

\% Atualizações:

$\mathbf{w}_{1}(n)=\mathbf{w}_{1}(n-1)+\mu_{1} \varepsilon_{1}(n) \mathbf{u}(n)$
$\operatorname{dis}(\mathbf{u}(n), \mathbf{C}(n))=\min _{1 \leq i \leq N_{\mathbf{C}}(n)}\left\|\mathbf{u}(n)-\mathbf{u}\left(c_{i}\right)\right\|$

Se $\operatorname{dis}(\mathbf{u}(n), \mathbf{C}(n)) \leq \rho$ :

$\mathbf{C}(n+1)=\mathbf{C}(n)$

$i^{*}=\arg \min _{1 \leq i \leq N_{\mathbf{C}}(n)}\left\|\mathbf{u}(n)-\mathbf{u}\left(c_{i}\right)\right\|$

$b_{i^{*}}(n)=b_{i^{*}}(n-1)+\mu e_{d 2}(n)$

\section{Caso contrário:}

$$
\begin{aligned}
& \mathbf{C}(n+1)=\{\mathbf{C}(n) \mathbf{u}(n)\} \\
& \mathbf{b}(n)=\left[\begin{array}{lll}
\mathbf{b}(n-1) & \mu e_{d 2}(n)
\end{array}\right] \\
& \varphi^{\prime}[\alpha(n-1)]=\frac{\operatorname{sgm}[\alpha(n-1)]\{1-\operatorname{sgm}[\alpha(n-1)]\}}{\operatorname{sgm}\left[\alpha^{+}\right]-\operatorname{sgm}\left[-\alpha^{+}\right]} \\
& p(n)=\eta p(n-1)+(1-\eta)\left[y_{1}(n)-y_{2}(n)\right]^{2} \\
& \alpha(n)=\alpha(n-1)+\frac{\mu_{\alpha}}{p(n)} e_{d}(n)\left[y_{1}(n)-y_{2}(n)\right] \varphi^{\prime}[\alpha(n-1)]
\end{aligned}
$$

Fim

Fonte: Autora. 


\subsubsection{Resultados de simulações}

Nas simulações foram considerados diferentes cenários baseados no modelo de canal representado na Figura 3 (página 8). A Tabela 7 contém a descrição de cada um desses cenários de simulação, em que $N$ é o número total de iterações e o sinal transmitido é uma sequência assumida iid. Os canais não lineares descritos são baseados em um modelo de canal não linear frequentemente encontrado em canais de satélite (KECHRIOTIS; ZERVAS; MANOLAKOS, 1994). Assim, foram verificados os resultados da combinação do CMA com o DFE-NLMS-DD e do CMA com o QKLMS-DD. Em ambos os casos, foi utilizado o erro de decisão global $e_{d}(n)$ para atualizar o parâmetro de mistura. Os resultados da combinação são comparados com os resultados das respectivas versões supervisionadas do equalizador DD, isto é, o DFE-NLMS e o QKLMS. Para cada simulação são mostrados em uma mesma figura os resultados das saídas $y_{1}(n), y_{2}(n), y(n)$ e $y_{\mathrm{DFE}}(n)$ ou $y_{\mathrm{QKLMS}}(n)$. O MSE e o valor médio do parâmetro de mistura $\mathrm{E}\{\lambda(n)\}$ foram obtidos com uma média de 100 realizações. Para facilitar a visualização, as curvas de MSE ainda foram suavizadas utilizando um filtro de média móvel de 128 coeficientes. Nas simulações que utilizam o QKLMS, mostra-se também o gráfico com as iterações em que há armazenamento de vetores no dicionário. Nesses casos, tanto o QKLMS como a combinação com o QKLMS-DD apresentam gráficos idênticos, pois o armazenamento no dicionário só depende do vetor de entrada $\mathbf{u}(n)$ e do limiar $\rho$. Por fim, em uma figura separada, são mostradas a ocorrência de erro entre o sinal transmitido com atraso $a(n-\Delta)$ e a saída decidida $\hat{a}(n-\Delta)$ para o CMA, a combinação e o DFE-NLMS/QKLMS.

A Figura 24 é referente a combinação do CMA com o DFE-NLMS-DD para o Cenário 2-A, em que são utilizados $\mu_{1}=10^{-7}, \mu_{2}=\mu_{D F E}=10^{-2}, \mu_{\alpha}=2,0 \times 10^{-2}, \alpha^{+}=4$ e os números de coeficientes dos filtros são $M_{1}=7, M_{2 f}=7$ e $M_{2 b}=5$. Na primeira metade das iterações, o canal apresenta uma não linearidade, e como esperado, a saída $y_{1}(n)$ do CMA é muito ruim. Já as saídas $y_{2}(n), y(n)$ e $y_{D F E}(n)$ aparentam ser melhores, uma vez que é possível verificar a separação dos níveis do sinal, excetuando-se a região entre -1 e 1 . O valor de $\operatorname{E}\{\lambda(n)\}$ é próximo a 1 somente no início indicando que o algoritmo CMA apresenta menor MSE nessas iterações. Nas demais iterações, $\mathrm{E}\{\lambda(n)\} \approx 0$, indicando que a combinação chaveou para o DFE-NLMS-DD. Na segunda metade das iterações o canal perde a não linearidade e os algoritmos simulados passam a apresentar um desempenho melhor em termos de MSE. No geral, a combinação apresentou desempenho muito próximo ao do DFE-NLMS supervisionado. 
Tabela 7: Cenários utilizados nas simulações com combinação convexa do CMA com o DFE-NLMS-DD e/ou CMA com QKLMS-DD.

\section{Cenário 2-A}

Sinal 8-PAM

Canal não linear: de 0 a $N / 2$ iterações:

$$
\begin{aligned}
& H(z)=0,2+z^{-1}+0,2 z^{-2} \\
& u(n)=x(n)+0,005 x^{2}(n)+0,005 x^{3}(n)
\end{aligned}
$$

Canal linear: de $N / 2+1$ a $N$ iterações:

$$
H(z)=0,2+z^{-1}+0,2 z^{-2}
$$

\section{Cenário 2-B}

Sinal 8-PAM

Canal não linear: de 0 a $N / 2$ iterações:

$$
\begin{aligned}
& H(z)=0,2+z^{-1}+0,2 z^{-2} \\
& u(n)=x(n)+0,01 x^{2}(n)+0,01 x^{3}(n)
\end{aligned}
$$

Canal linear: de $N / 2+1$ a $N$ iterações:

$$
H(z)=0,2+z^{-1}+0,2 z^{-2}
$$

\section{Cenário 2-C}

Sinal 4-PAM

Canal não linear:

$$
\begin{aligned}
& H(z)=0,2+z^{-1}+0,2 z^{-2} \\
& u(n)=x(n)+0,2 x^{2}(n)+0,1 x^{3}(n)
\end{aligned}
$$

\section{Cenário 2-D}

Sinal 4-PAM

Canal não linear variante no tempo:

$$
\begin{aligned}
& H(z, n)=\frac{0,4}{N} n+z^{-1}+\frac{0,4}{N} n z^{-2} \\
& u(n)=x(n)+0,2 x^{2}(n)+0,05 x^{3}(n)
\end{aligned}
$$

\section{Cenário 2-E}

\section{Sinal 4-PAM}

Canal linear com nulo espectral:

$$
H(z)=0,5+z^{-1}+0,5 z^{-2}
$$

Fonte: Autora. 
Figura 24 - Exemplo de combinação convexa de um equalizador CMA com um DFE-NLMS-DD para o Cenário 2-A da Tabela 7 (página 50). Em que se usa o erro de decisão global $e_{d}(n), \mu_{1}=10^{-7}$, $\mu_{2}=\mu_{\mathrm{DFE}}=10^{-2}, \mu_{\alpha}=2,0 \times 10^{-2}, \alpha^{+}=4, M_{1}=7, M_{2 f}=7$ e $M_{2 b}=5$. O MSE e E $\{\lambda(n)\}$ são calculados para 100 realizações.

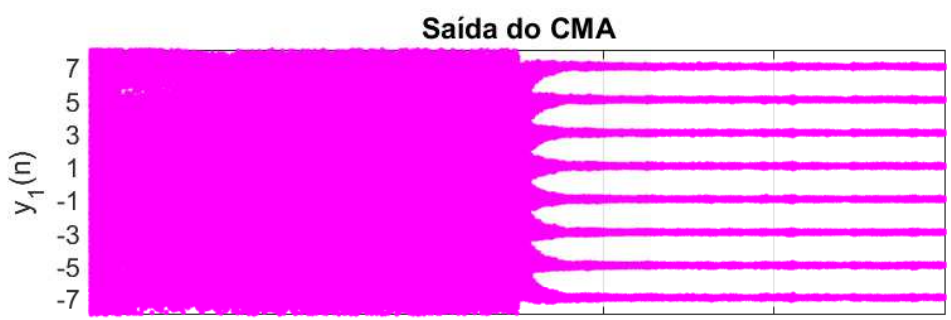

Saída do DFE-NLMS-DD

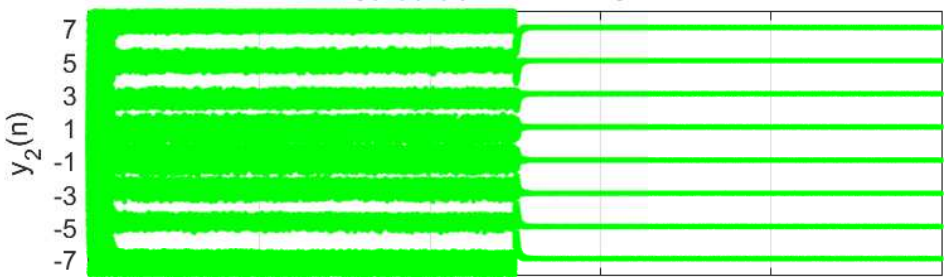

Saída global da combinação

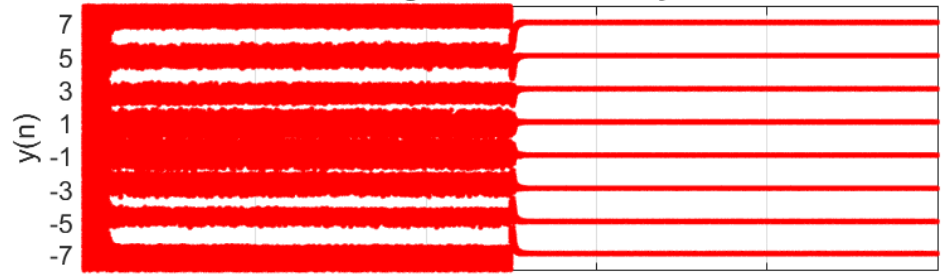

Saída do DFE-NLMS supervisionado
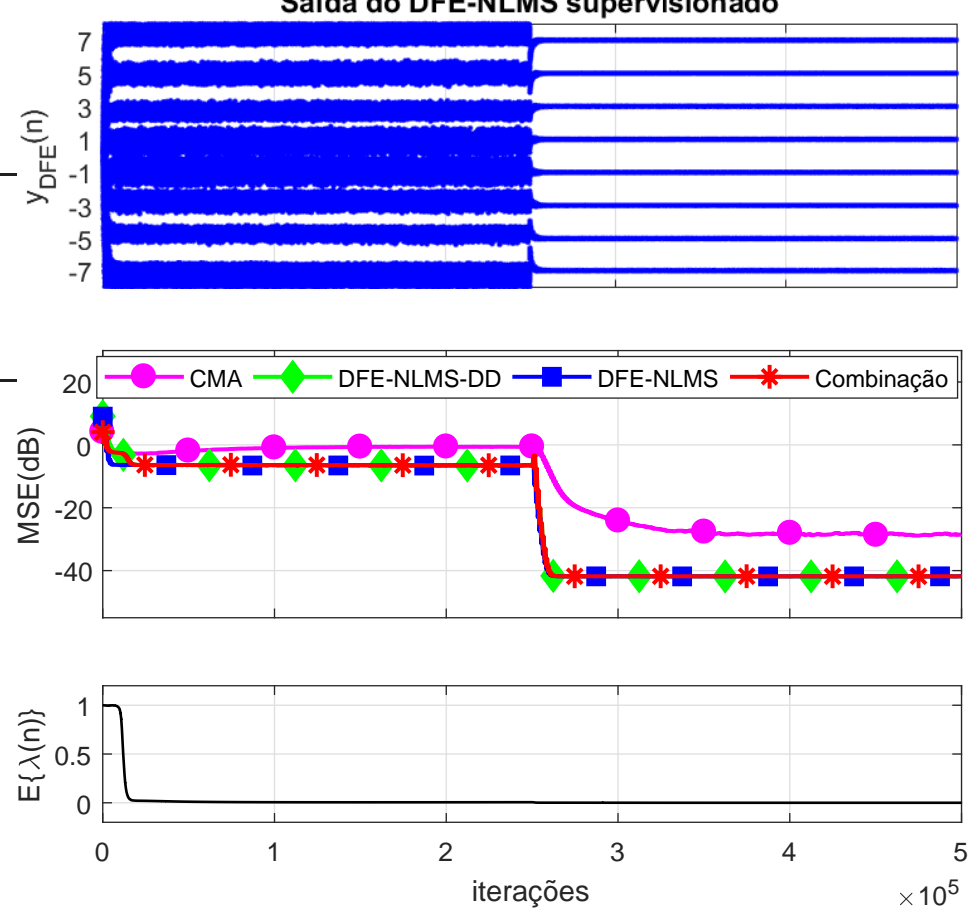

Fonte: Autora 
A Figura 25 mostra os gráficos que representam (por meio de raias) as iterações que apresentam erros entre o sinal transmitido $a(n-\Delta)$ e o sinal de saída decidido $\hat{a}(n-\Delta)$ para o CMA, combinação e DFE-NLMS supervisionado. Esses gráficos mostram que quando o canal é não linear os erros no CMA são mais frequentes que na combinação e no DFE-NLMS supervisionado, sendo nulos para todos os casos quando o canal é linear.

Figura 25 - Diferença entre o sinal transmitido com atraso $a(n-\Delta)$ e o sinal decidido $\hat{a}(n-\Delta)$, para a combinação do CMA com o DFE-NLMS-DD, como minimização do erro de decisão global ao quadrado $e_{d}^{2}(n)$, dado o Cenário 2-A da Tabela 7 (página 50).

CMA: Taxa de Erro $=1,3 \times 10^{-1}$

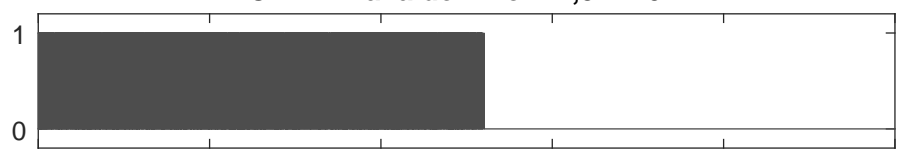

Combinação CMA xDFE-NLMS-DD: Taxa de Erro $=6,1 \times 10^{-3}$

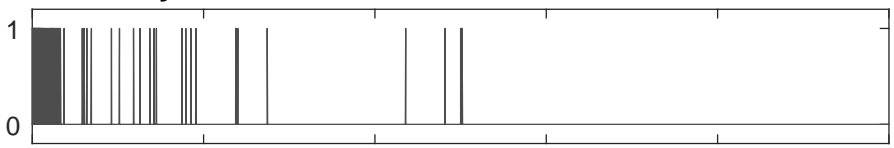

DFE-NLMS supervisionado: Taxa de Erro $=3,0 \times 10^{-3}$

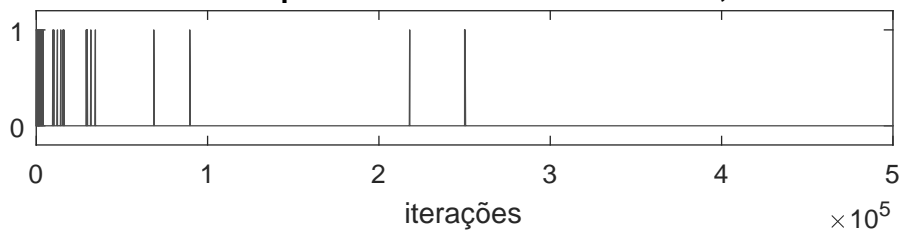

Fonte: Autora.

Ainda para o cenário 2-A, a Figura 26 mostra os resultados da simulação para a combinação do CMA com o QKLMS-DD, em que são utilizados $\mu_{1}=10^{-7}, \mu_{2}=\mu_{\mathrm{QKLMS}}=0,2, \mu_{\alpha}=$ $10^{-2}, \alpha^{+}=4, \sigma=15, \rho=80$ e os números de coeficientes dos filtros são $M_{1}=7$ e $M_{2}=M_{\mathrm{QKLMS}}=5$. Na primeira metade das iteraç̃̃es, quando o canal é não linear, verifica-se que no início da simulação a combinação se comporta como o CMA, mas depois de aproximadamente $n=2,8 \times 10^{4}$ iterações ela chaveia para o QKLMS-DD e $\operatorname{E}\{\lambda(n)\} \approx 0$. Em outras palavras, a combinação segue o QKLMS-DD que apresenta um MSE menor. Além disso, o MSE da combinação se aproxima do MSE do QKLMS supervisionado, mostrando que este esquema de combinação pode ser uma boa alternativa para equalização cega de canais não lineares. 
Figura 26 - Exemplo de combinação convexa de um equalizador CMA com um QKLMS-DD para o Cenário 2-A da Tabela 7 (página 50). Em que se usa o erro de decisão global $e_{d}(n), \mu_{1}=10^{-7}$, $\mu_{2}=\mu_{\mathrm{QKLMS}}=0,2, \mu_{\alpha}=10^{-2}, M_{1}=7, M_{2}=M_{\mathrm{QKLMS}}=5, \sigma=15, \rho=80$ e $\alpha^{+}=4 . \mathrm{O} \mathrm{MSE}$ e $\mathrm{E}\{\lambda(n)\}$ são calculados para 100 realizações.
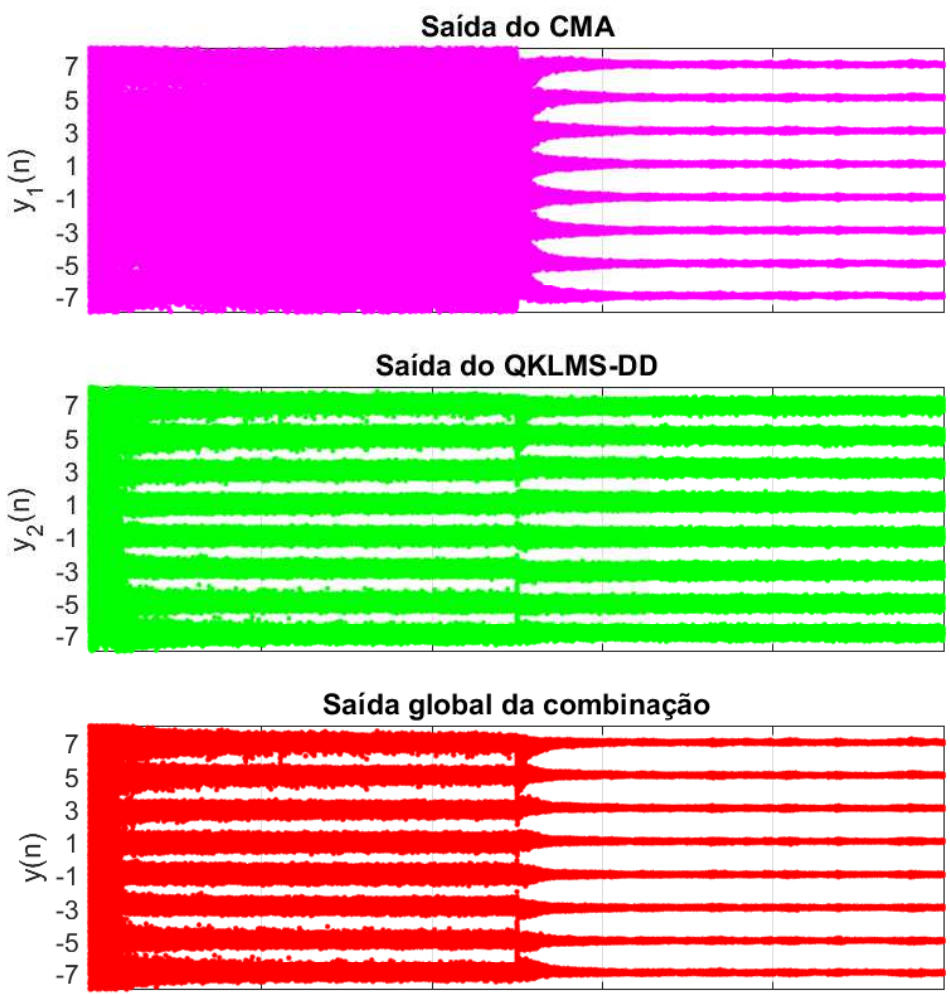

Saída do QKLMS supervisionado
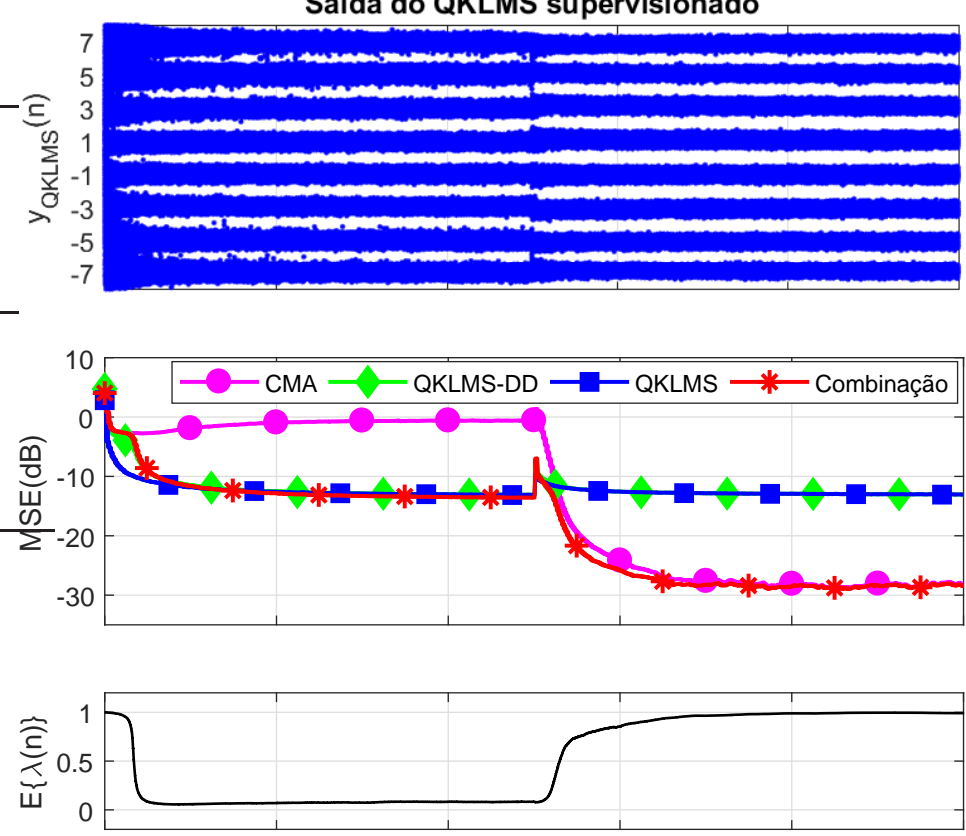

Atualizações no dicionário: 174

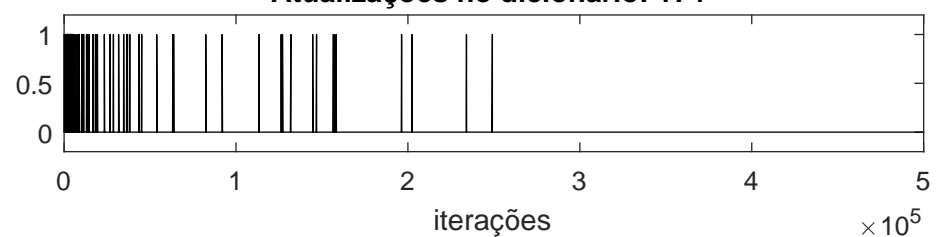

Fonte: Autora. 
Quando o canal torna-se linear, após a iteração $n=2,5 \times 10^{5}$, o CMA passa a funcionar melhor que o QKLMS-DD, o que faz com que $\mathrm{E}\{\lambda(n)\} \approx 1$ e a combinação chaveie para essa solução. Isso acontece porque o CMA é mais apropriado que o QKLMS para a equalização de canais lineares. O último gráfico da Figura 26, que representa o armazenamento de vetores no dicionário $\mathrm{C}$, mostra que o QKLMS armazena mais vetores durante a convergência do algoritmo. Ao todo, foram armazenados 174 vetores, número muito inferior a $5 \times 10^{5}$, que seria o tamanho do dicionário caso o algoritmo KLMS fosse utilizado.

A Figura 27 mostra que a ocorrência de erro entre o sinal transmitido $a(n-\Delta)$ e o sinal de saída decidido $\hat{a}(n-\Delta)$ é muito frequente no CMA, enquanto na combinação e no QKLMS-DD supervisionado os erros são mais frequentes no início das iterações, diminuindo após a convergência desses algoritmos. Quando o canal é linear, esses erros tornam-se nulos para todos os algoritmos.

Figura 27 - Diferença entre o sinal transmitido com atraso $a(n-\Delta)$ e o sinal decidido $\hat{a}(n-\Delta)$, para a combinação do CMA com o QKLMS-DD, como minimização do erro de decisão global ao quadrado $e_{d}^{2}(n)$, dado o Cenário 2-A da Tabela 7 (página 50).

CMA: Taxa de Erro $=1,3 \times 10^{-1}$

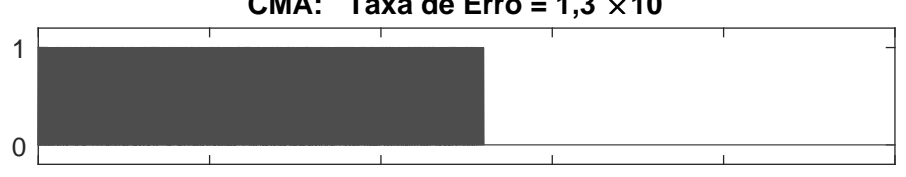

Combinação CMA $\times$ QKLMS-DD: Taxa de Erro = 6,2 $\times 10^{-3}$

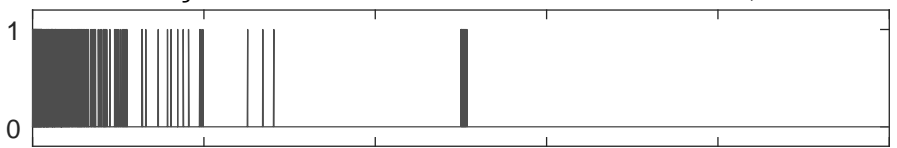

QKLMS supervisionado: Taxa de Erro $=1,2 \times 10^{-3}$

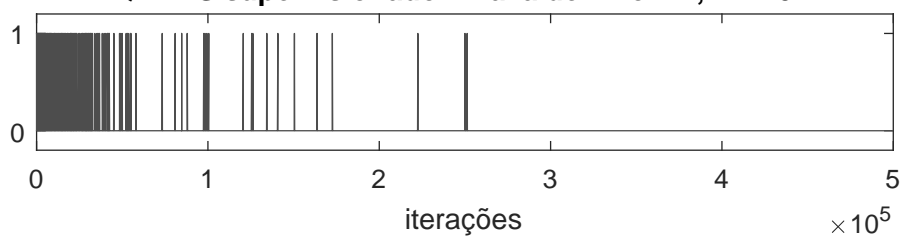

Fonte: Autora. 
Comparando a Figura 25 com a Figura 27, verifica-se que a combinação com DFE-NLMSDD tem um desempenho similar ao da combinação com QKLMS-DD em termos de erro após a decisão. Assim o erro existente entre os símbolos -1 e +1 do DFE acabam sendo "equivalentes" aos erros iniciais da convergência do QKLMS-DD.

O Cenário 2-B é semelhante ao 2-A, a única diferença é que a não linearidade do canal na primeira metade das iterações é mais severa. A Figura 28 mostra os resultados da simulação para a combinação do CMA com o DFE-NLMS-DD. Os parâmetros utilizados nesse caso foram: $\mu_{1}=10^{-8}, \mu_{2}=\mu_{D F E}=10^{-1}, \mu_{\alpha}=1,5 \times 10^{-3}, \alpha^{+}=4$ e os números de coeficientes dos filtros são $M_{1}=7, M_{2 f}=7$ e $M_{2 b}=5$.

Como esperado, o desempenho do CMA é muito ruim devido à não linearidade do canal. Da mesma forma, os desempenhos da combinação, do DFE-NLMS-DD e do DFE-NLMS supervisionado também foram ruins, o que pode ser justificado por uma limitação do DFE em lidar com canais com não linearidade mais severa. Observando os sinais de saída e o MSE de cada algoritmo, nota-se que quando o canal torna-se linear, o CMA melhora seu desempenho aos poucos, o que faz o MSE do DFE-NLMS-DD diminuir para um patamar próximo ao do DFE-NLMS supervisionado, em torno de $-40,0 \mathrm{~dB}$. Com isso, $\mathrm{E}\{\lambda(n)\}$ aproxima-se de 0 , assim como o MSE da combinação aproxima-se do mesmo patamar do DFE-NLMS-DD, o que mostra que a combinação chaveia para o modo DD nas iterações finais.

A Figura 29 ilustra bem o desempenho dessa simulação, uma vez que no canal não linear os erros nas estimativas recuperadas são muito frequentes e no canal linear esses erros são praticamente nulos. 
Figura 28 - Exemplo de combinação convexa de um equalizador CMA com um DFE-NLMS-DD para o Cenário 2-B da Tabela 7 (página 50). Em que se usa o erro de decisão global $e_{d}(n), \mu_{1}=10^{-8}$, $\mu_{2}=\mu_{\mathrm{DFE}}=10^{-1}, \mu_{\alpha}=1,5 \times 10^{-3}, M_{1}=7, M_{2 f}=7, M_{2 b}=5$ e $\alpha^{+}=4$. O MSE e E $\{\lambda(n)\}$ são calculados para 100 realizações.

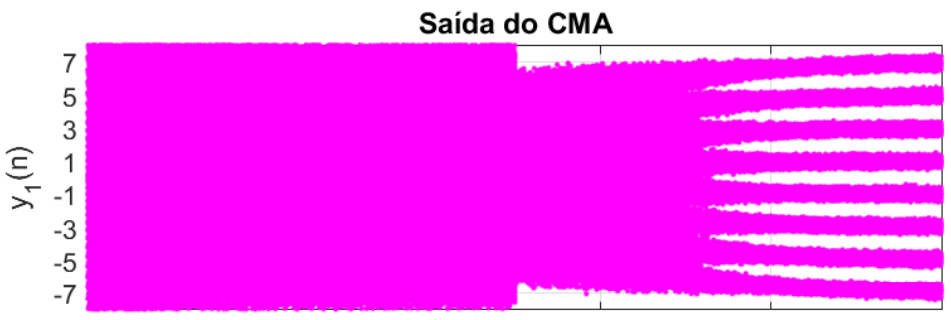

Saída do DFE-NLMS-DD

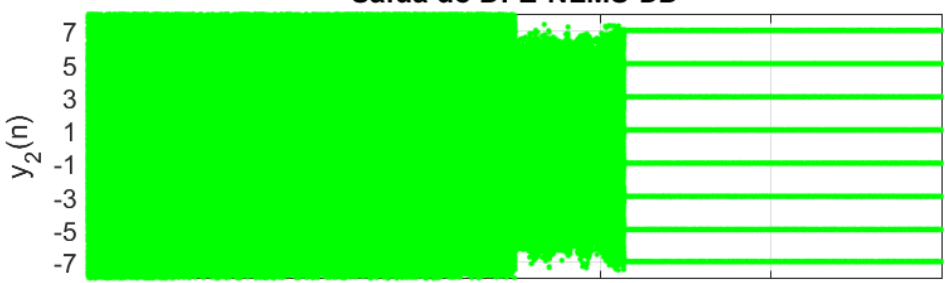

Saída global da combinação

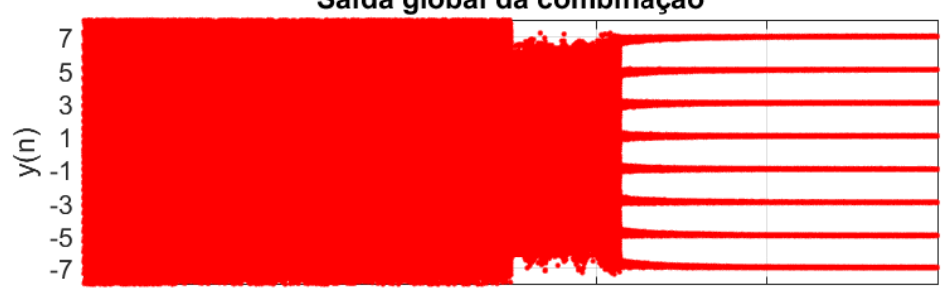

Saída do DFE-NLMS supervisionado
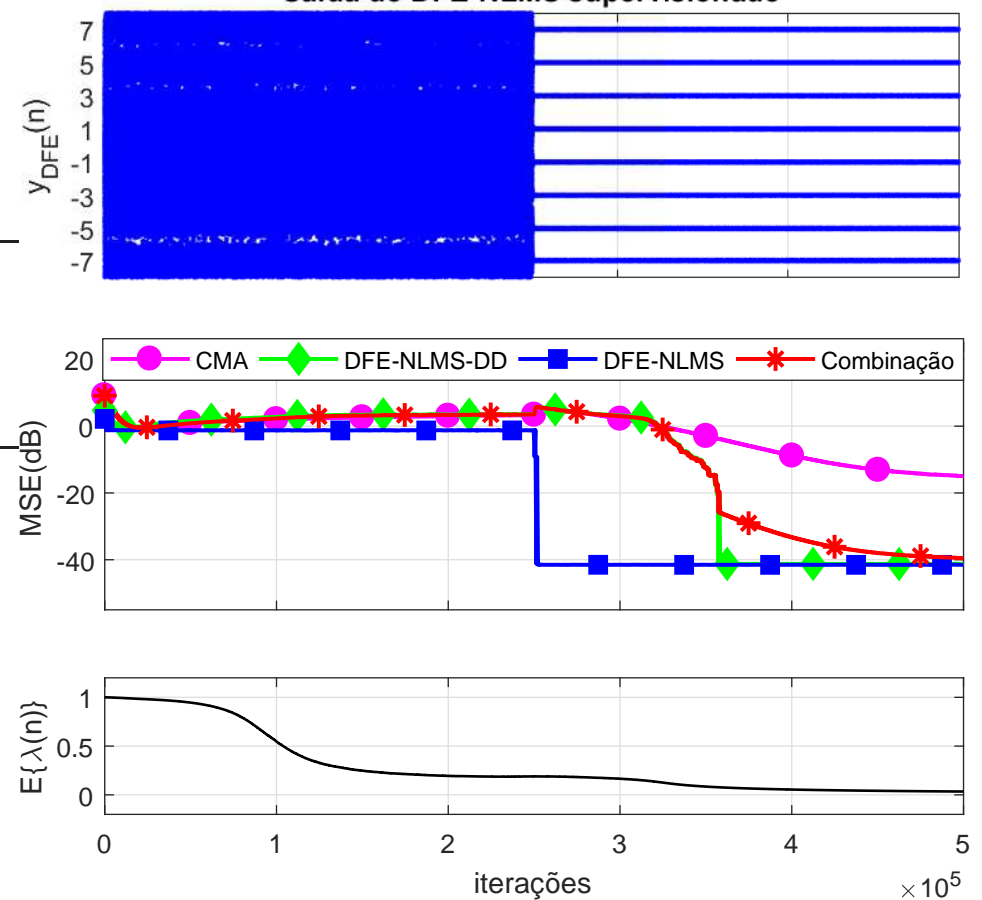

Fonte: Autora. 
Figura 29 - Diferença entre o sinal transmitido com atraso $a(n-\Delta)$ e o sinal decidido $\hat{a}(n-\Delta)$, para a combinação do CMA com o DFE-NLMS-DD, como minimização do erro de decisão global ao quadrado $e_{d}^{2}(n)$, dado o Cenário 2-B da Tabela 7 (página 50).

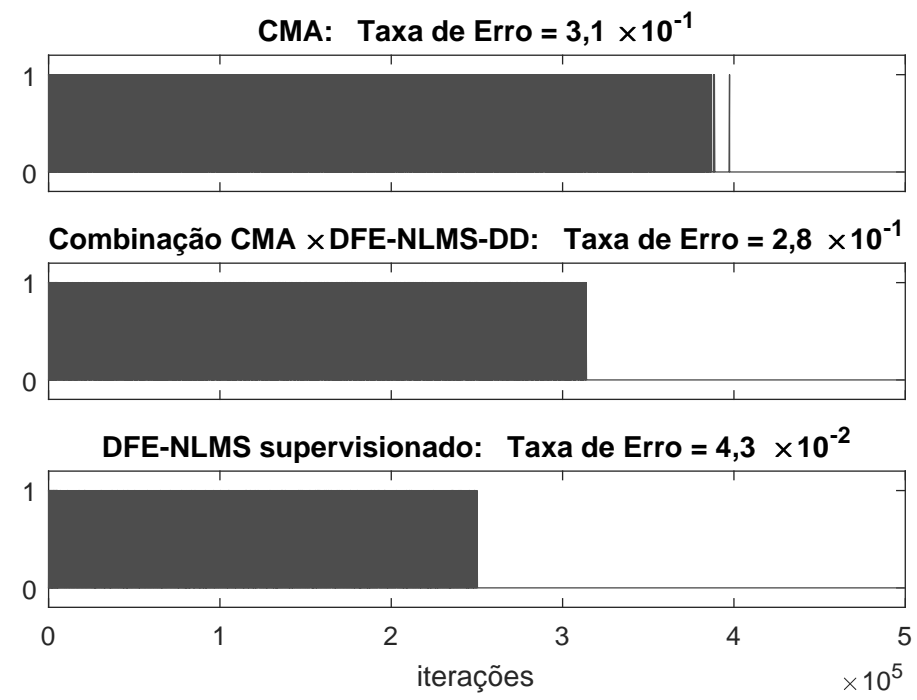

Fonte: Autora.

Na Figura 30, são mostrados os resultados da combinação do CMA com o QKLMS-DD considerando o Cenário 2- $\mathrm{B}$, onde foram utilizados os seguintes parâmetros: $\mu_{1}=2,0 \times 10^{-8}$, $\mu_{2}=\mu_{\mathrm{QKLMS}}=0,4, \mu_{\alpha}=3,0 \times 10^{-2}, \alpha^{+}=4, \sigma=15, \rho=80$, sendo os números de coeficientes dos filtros iguais a $M_{1}=7$ e $M_{2}=M_{\mathrm{QKLMS}}=5$.

Durante as iteraç̃̃es em que o canal é não linear, o desempenho do CMA apesar de ruim, consegue fazer o desempenho do QKLMS-DD melhorar e a combinação chavear para essa solução, com $\mathrm{E}\{\lambda(n)\} \approx 0$. Nesse caso, o MSE da combinação é igual a $-11,4 \mathrm{~dB}$, ficando muito próximo ao MSE do QKLMS supervisionado. Quando o canal torna-se linear, o MSE do CMA diminui e $\mathrm{E}\{\lambda(n)\}$ se aproxima de 1, fazendo a combinação chavear pra o CMA. O MSE do QKLMS-DD e do supervisionado permanecem em $-11,4 \mathrm{~dB}$, mostrando que essas soluções não são as mais adequadas para os canais lineares. Assim, quando o canal é não linear o desempenho da combinação é aproximado ao do QKLMS-DD e quando o canal é linear o desempenho é aproximado ao do CMA. O último gráfico da Figura 30 mostra que o armazenamento de vetores no dicionário, ao todo 403, é maior no início da convergência e quando o canal é linear o dicionário deixa de ser atualizado, pois a degradação do canal diminui assim como a distância entre os novos vetores de entrada e os presentes no dicionário. 
Figura 30 - Exemplo de combinação convexa de um equalizador CMA com um QKLMS-DD para o Cenário 2-B da Tabela 7 (página 50). Em que se usa o erro de decisão global $e_{d}(n), \mu_{1}=2,0 \times 10^{-8}$, $\mu_{2}=\mu_{\mathrm{QKLMS}}=0,4, \mu_{\alpha}=3,0 \times 10^{-2}, M_{1}=7, M_{2}=M_{\mathrm{QKLMS}}=5, \sigma=15, \rho=80$ e $\alpha^{+}=4 . \mathrm{O}$ MSE e $\mathrm{E}\{\lambda(n)\}$ são calculados para 100 realizações.

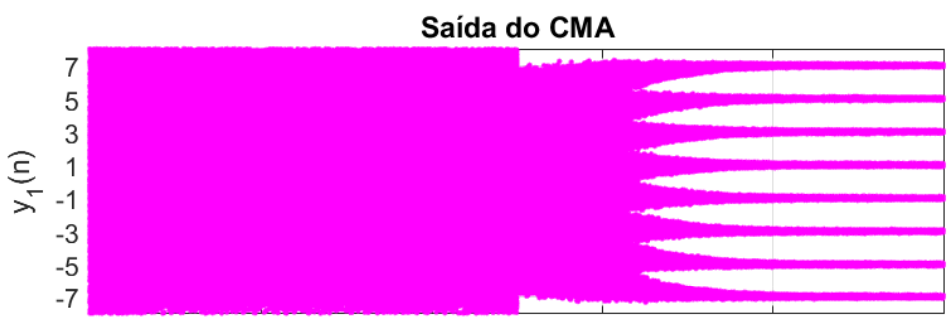

Saída do QKLMS-DD

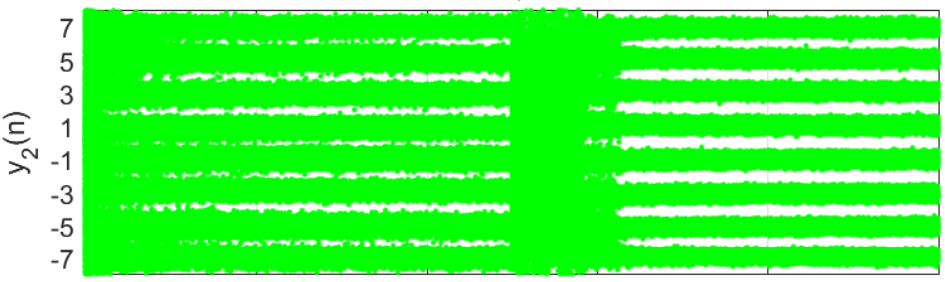

Saída da combinação

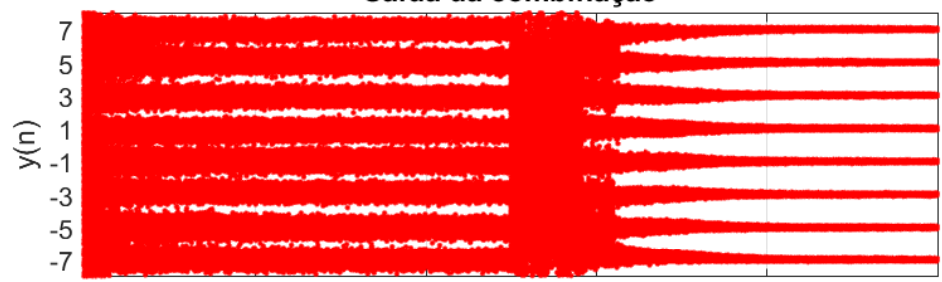

Saída do QKLMS supervisionado
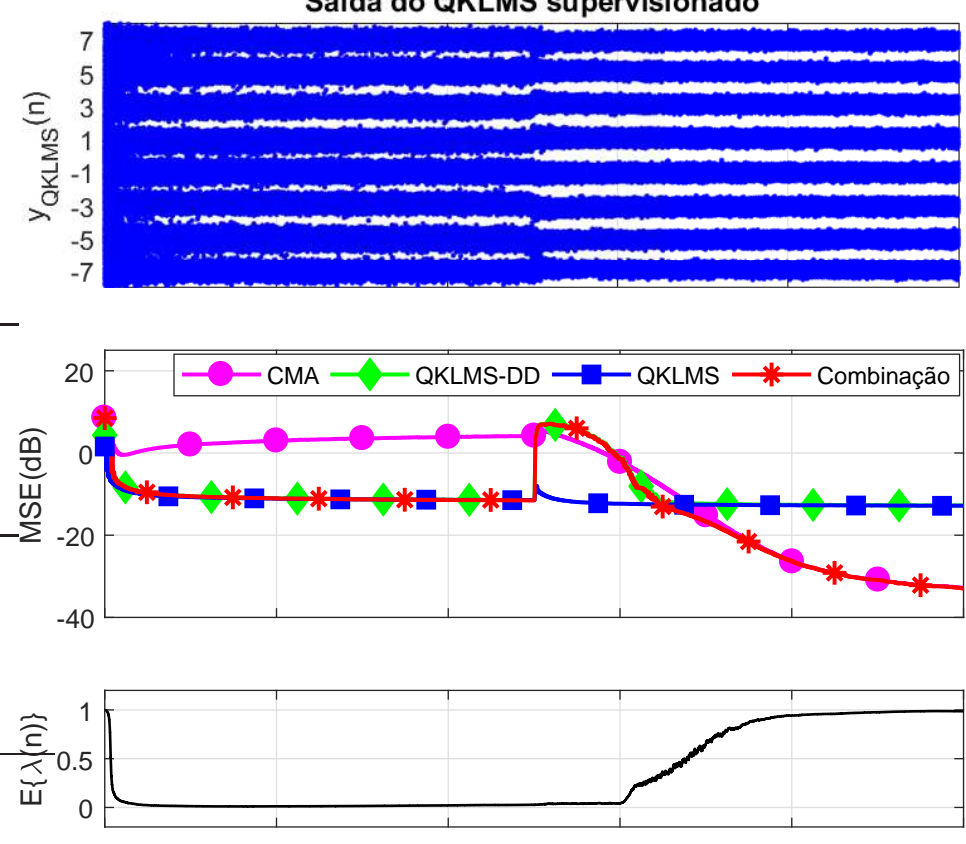

Atualizações no dicionário: 403

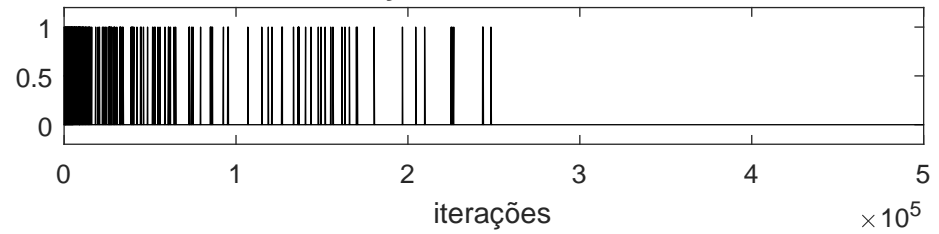

Fonte: Autora. 
Assim como na Figura 27, a Figura 31 mostra que quando o canal é não linear os erros entre o sinal transmitido $a(n-\Delta)$ e o sinal de saída decidido $\hat{a}(n-\Delta)$ são frequentes para o CMA, enquanto para a combinação e o QKLMS supervisionado os erros diminuem com a convergência dos algoritmos. E quando o canal torna-se linear, os erros tornam-se nulos com a convergência de todos os algoritmos.

Figura 31 - Diferença entre o sinal transmitido com atraso $a(n-\Delta)$ e o sinal decidido $\hat{a}(n-\Delta)$, para a combinação do CMA com o QKLMS-DD, como minimização do erro de decisão global ao quadrado $e_{d}^{2}(n)$, dado o Cenário 2-B da Tabela 7 (página 50).

CMA: Taxa de Erro $=2,8 \times 10^{-1}$

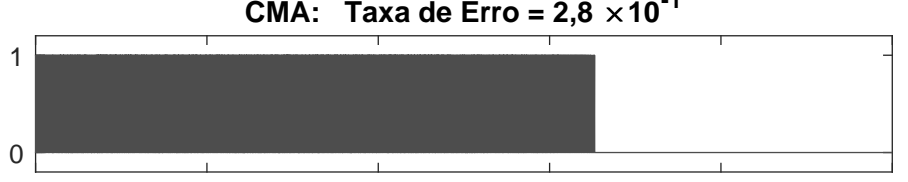

Combinação CMA $\times$ QKLMS-DD: Taxa de Erro = 6,2 × $\times 10^{-2}$

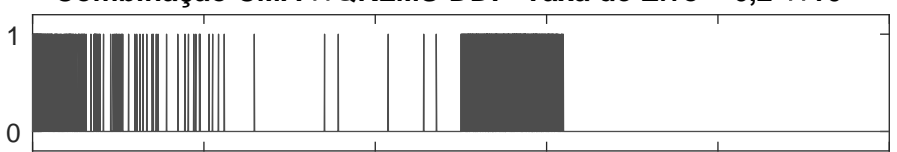

QKLMS Supervisionado: Taxa de Erro $=1,1 \times 10^{-3}$

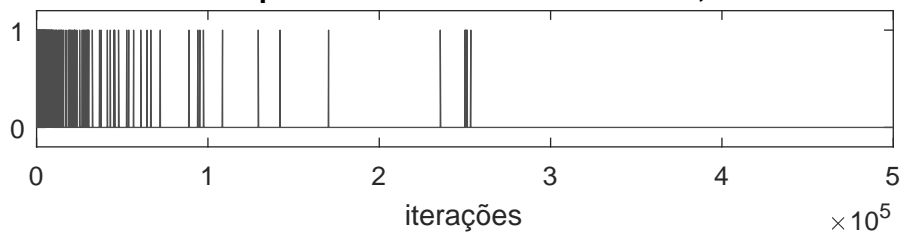

Fonte: Autora.

No Cenário 2-C, o canal de comunicação é fixo ao longo das $N=10^{5}$ iterações e possui uma não linearidade mais severa que as dos Cenários 2-A e 2-B. Nesse caso, as Figuras 32 e 33 mostram que tanto a combinação do CMA com o DFE-NLMS-DD como o DFE-NLMS supervisionado apresentam um desempenho muito ruim para equalização. Os parâmetros usados são $\mu_{1}=2,0 \times 10^{-7}, \mu_{2}=\mu_{\mathrm{DFE}}=5,0 \times 10^{-2}, \mu_{\alpha}=10^{-1}, \alpha^{+}=4$, e os números de coeficientes dos filtros são $M_{1}=7, M_{2 f}=7$ e $M_{2 b}=5$. 
Figura 32 - Exemplo de combinação convexa de um equalizador CMA com um DFE-NLMS-DD para o Cenário 2-C da Tabela 7 (página 50). Em que se usa o erro de decisão global $e_{d}(n), \mu_{1}=2,0 \times 10^{-7}$, $\mu_{2}=\mu_{\mathrm{DFE}}=5,0 \times 10^{-2}, \mu_{\alpha}=10^{-1}, M_{1}=7, M_{2 f}=7, M_{2 b}=5$ e $\alpha^{+}=4$. O MSE e E $\{\lambda(n)\}$ são calculados para 100 realizações.
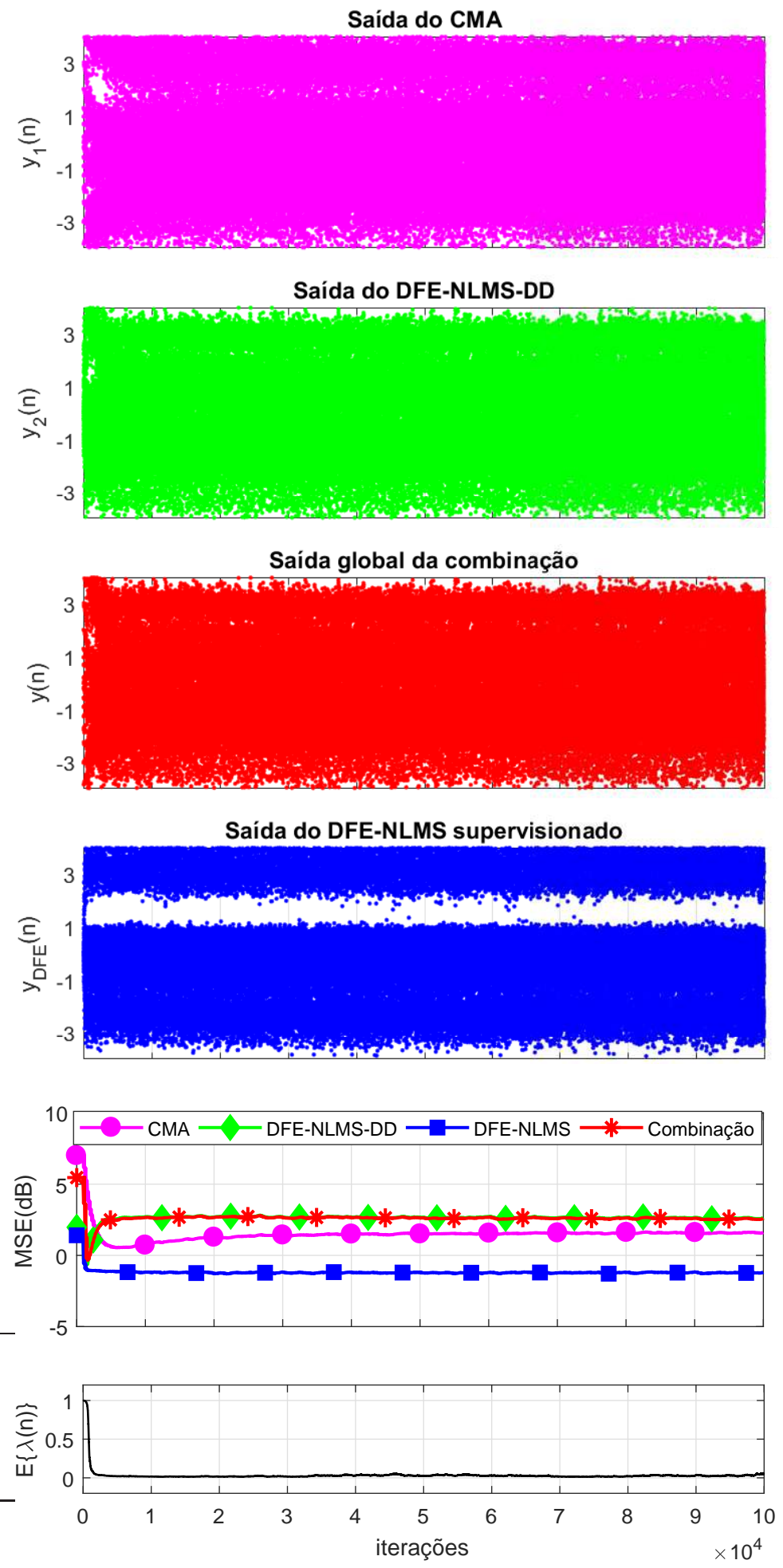

Fonte: Autora. 
Figura 33 - Diferença entre o sinal transmitido com atraso $a(n-\Delta)$ e o sinal decidido $\hat{a}(n-\Delta)$, para a combinação do CMA com o DFE-NLMS-DD, como minimização do erro de decisão global ao quadrado $e_{d}^{2}(n)$, dado o Cenário 2-C da Tabela 7 (página 50).

CMA: Taxa de Erro $=3,8 \times 10^{-1}$

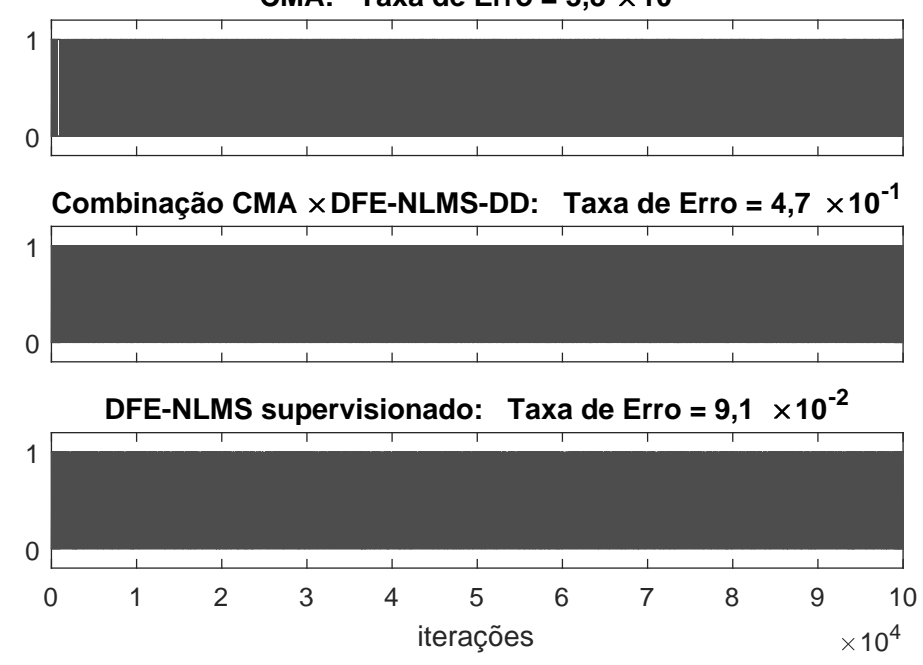

Fonte: Autora.

A Figura 34 mostra os resultados da combinação do CMA com o QKLMS-DD para o Cenário 2-C ao longo de $N=10^{5}$ iterações, em que são utilizados os seguintes parâmetros: $\mu_{1}=2,0 \times 10^{-7}, \mu_{2}=\mu_{\mathrm{QKLMS}}=10^{-1}, \mu_{\alpha}=10^{-1}, \alpha^{+}=4, \sigma=8, \rho=20$, e os números de coeficientes dos filtros iguais a $M_{1}=7$ e $M_{2}=M_{\mathrm{QKLMS}}=5$. Esses resultados mostram que mesmo com o desempenho pobre do CMA, a combinação chaveia para o QKLMS-DD quando $\mathrm{E}\{\lambda(n)\} \approx 0$, funcionando com desempenho semelhante ao do QKLMS supervisionado em termos de MSE. A atualização do dicionário diminui ao longo das iterações, sendo armazenados ao todo 478 vetores.

A Figura 35 mostra que a ocorrência de erro entre o sinal transmitido e as saídas decididas diminui com as iterações, em uma taxa mais lenta para a combinação em comparação ao QKLMS supervisionado, enquanto o CMA não consegue obter trechos sem erros. 
Figura 34 - Exemplo de combinação convexa de um equalizador CMA e com um QKLMS-DD para o Cenário 2-C da Tabela 7 (página 50). Em que se usa o erro de decisão global $e_{d}(n), \mu_{1}=2,0 \times 10^{-7}$, $\mu_{2}=\mu_{\mathrm{QKLMS}}=10^{-1}, \mu_{\alpha}=10^{-1}, M_{1}=7, M_{2}=M_{\mathrm{QKLMS}}=5, \sigma=8, \rho=20$ e $\alpha^{+}=4 . \mathrm{O} \mathrm{MSE}$ e $\mathrm{E}\{\lambda(n)\}$ são calculados para 100 realizações.
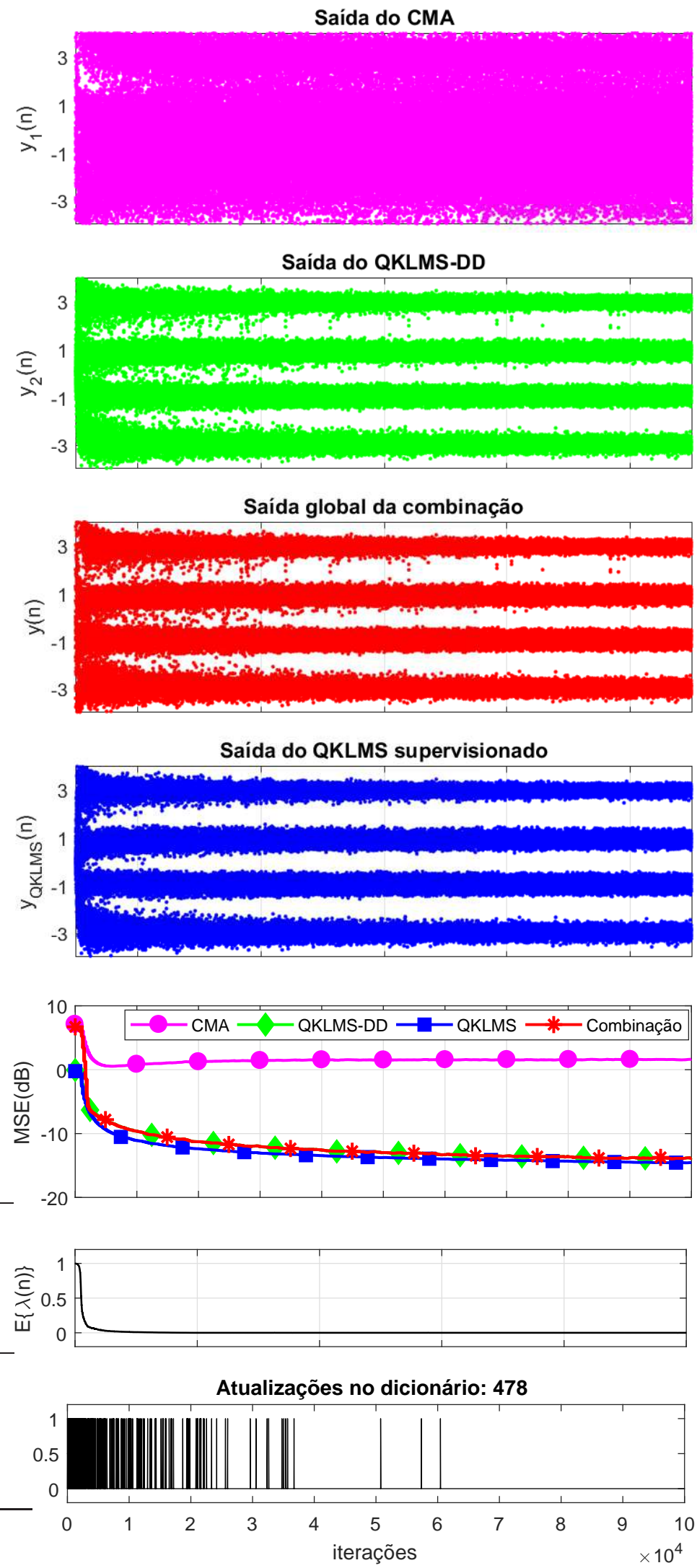

Fonte: Autora. 
Figura 35 - Diferença entre o sinal transmitido com atraso $a(n-\Delta)$ e o sinal decidido $\hat{a}(n-\Delta)$, para a combinação do CMA com o QKLMS-DD, como minimização do erro de decisão global ao quadrado $e_{d}^{2}(n)$, dado o Cenário 2-C da Tabela 7 (página 50).

CMA: Taxa de Erro $=3,8 \times 10^{-1}$

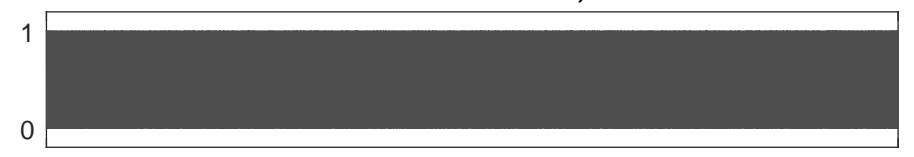

Combinação CMA $\times$ QKLMS-DD: Taxa de Erro $=4,3 \times 10^{-3}$

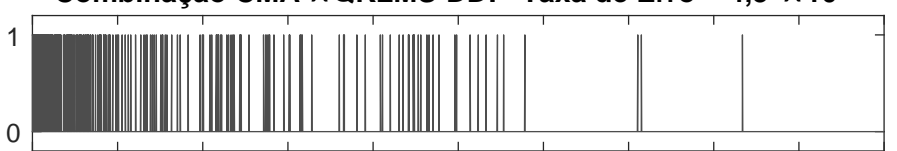

QKLMS supervisionado: Taxa de Erro $=3,7 \times 10^{-3}$

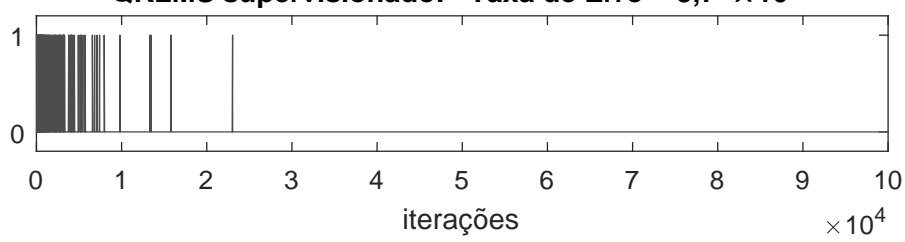

Fonte: Autora.

No cenário 2-D, o canal de comunicação é não linear variante e sua função de transferência é representada por $H(z, n)=k(n)+z^{-1}+k(n) z^{-2}$, em que $k$ varia linearmente de 0 a 0,4 ao longo das $N=5,0 \times 10^{5}$ iterações. A não linearidade é mantida constante ao longo das iterações. Parte-se de um caso em que a degradação ocorre só devido a não linearidade $(k(1)=0)$ e chega-se ao caso com degradação severa $(k(N)=0,4)$.

A Figura 36 mostra os resultados da simulação da combinação do CMA com o DFENLMS-DD, em que os parâmetros usados são $\mu_{1}=5,0 \times 10^{-7}, \mu_{2}=\mu_{\mathrm{DFE}}=5,0 \times 10^{-2}$, $\mu_{\alpha}=10^{-2}, \alpha^{+}=4$, e os números de coeficientes dos filtros são $M_{1}=7, M_{2 f}=15 \mathrm{e}$ $M_{2 b}=7$. Na Figura 36, tanto as saídas de cada algoritmo como suas respectivas curvas de MSE mostram que os desempenhos dessas soluções deterioram-se ao longo das iterações. Além disso, a Figura 37 mostra que os erros entre o sinal transmitido e a saída decidida são muito frequentes para todos os algoritmos. 
Figura 36 - Exemplo de combinação convexa de um equalizador CMA com um DFE-NLMS-DD para o Cenário 2-D da Tabela 7 (página 50). Em que se usa o erro de decisão global $e_{d}(n), \mu_{1}=5,0 \times 10^{-7}$, $\mu_{2}=\mu_{\mathrm{DFE}}=5,0 \times 10^{-2}, \mu_{\alpha}=10^{-2}, M_{1}=7, M_{2 f}=15, M_{2 b}=7$, e $\alpha^{+}=4$. O MSE e E $\{\lambda(n)\}$ são calculados para 100 realizações.
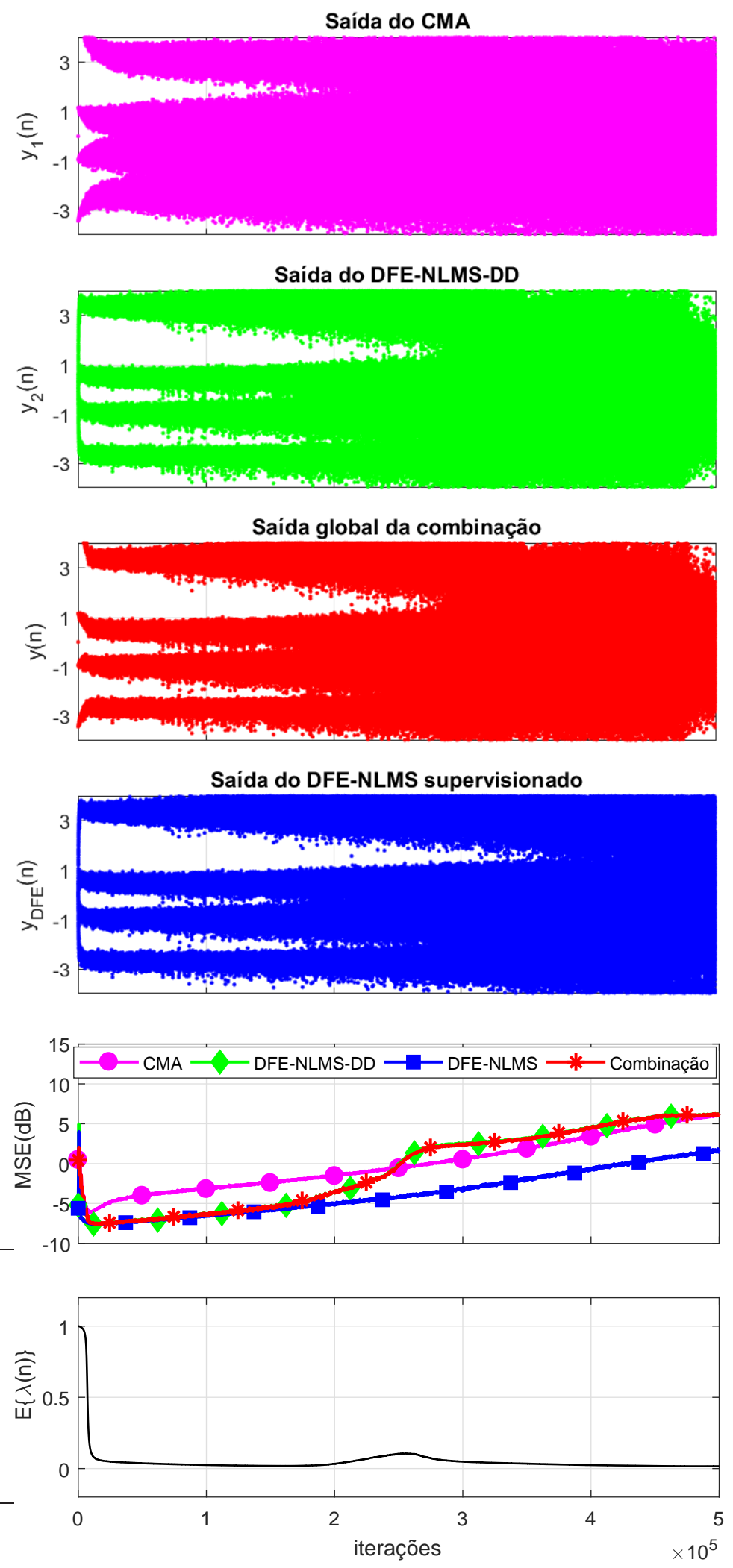

Fonte: Autora. 
Figura 37 - Diferença entre o sinal transmitido com atraso $a(n-\Delta)$ e o sinal decidido $\hat{a}(n-\Delta)$, para a combinação do CMA com o DFE-NLMS-DD, como minimização do erro de decisão global ao quadrado $e_{d}^{2}(n)$, dado o Cenário 2-D da Tabela 7 (página 50).

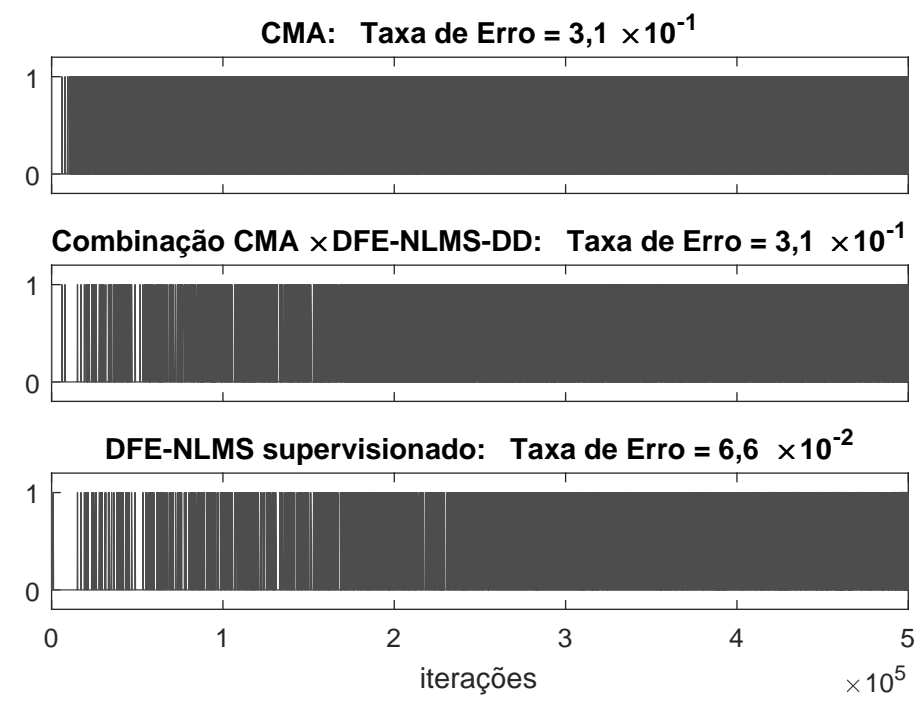

Fonte: Autora.

A Figura 38 mostra os resultados para a combinação do CMA com o QKLMS-DD para o Cenário 2-D, em que se utiliza $\mu_{1}=10^{-6}, \mu_{2}=\mu_{\mathrm{QKLMS}}=10^{-1}, \mu_{\alpha}=2,0 \times 10^{-2}, \alpha^{+}=4$, $\sigma=8, \rho=20$ e os números de coeficientes dos filtros são $M_{1}=7$ e $M_{2}=M_{\mathrm{QKLMS}}=5$. Apesar da saída do CMA ser muito ruim, as saídas da combinação e do QKLMS-DD são relativamente boas, sendo pioradas conforme a degradação do canal aumenta. No início das iterações, o MSE do CMA é o menor e $\mathrm{E}\{\lambda(n)\} \approx 1$, portanto a combinação utiliza o CMA para melhorar as estimativas do QKLMS-DD, fazendo com que a combinação chaveie para essa solução e $\mathrm{E}\{\lambda(n)\} \approx 0$ após aproximadamente $n=3660$ iterações. Assim, o MSE da combinação é menor que o do CMA e segue o MSE do QKLMS supervisionado, aumentando ao longo das iterações devido a piora do canal. O gráfico com as atualizações no dicionário mostra que conforme a degradação aumenta, mais vetores são incluídos. Isso ocorre devido ao aumento da distância entre os vetores novos e os já armazenados, que ao todo foram 637 . 
Figura 38 - Exemplo de combinação convexa de um equalizador CMA com um QKLMS-DD para o Cenário 2-D da Tabela 7 (página 50). Em que se usa o erro de decisão global $e_{d}(n), \mu_{1}=10^{-6}$, $\mu_{2}=\mu_{\mathrm{QKLMS}}=10^{-1}, \mu_{\alpha}=2,0 \times 10^{-2}, M_{1}=7, M_{2}=M_{\mathrm{QKLMS}}=5, \sigma=8, \rho=20$ e $\alpha^{+}=4$.

O MSE e $\mathrm{E}\{\lambda(n)\}$ são calculados para uma média de 100 realizações.
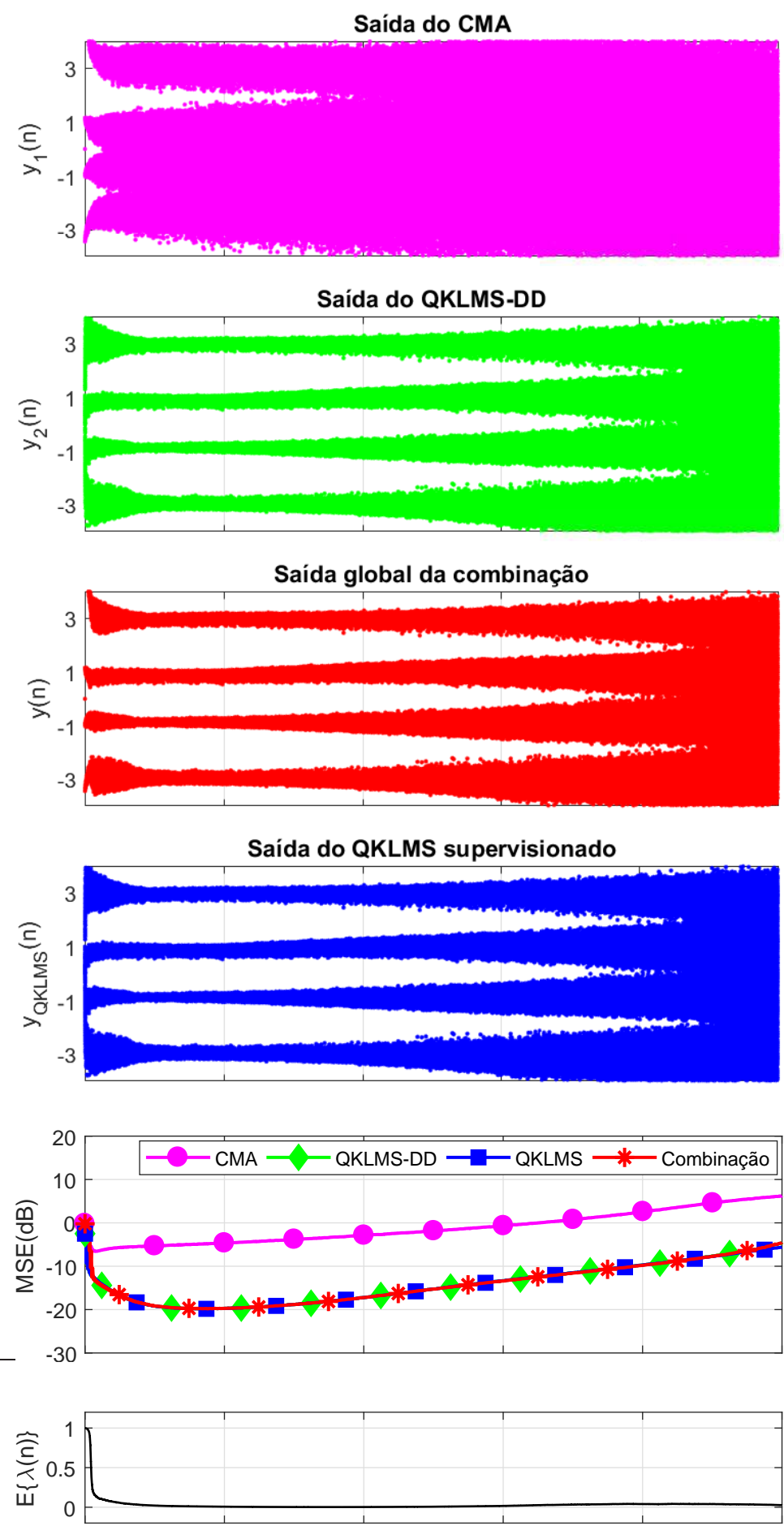

Atualizações no dicionário: 637

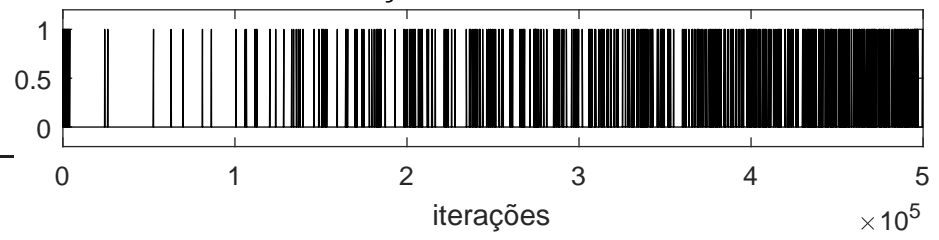

Fonte: Autora. 
A Figura 39 mostra que a ocorrência de erro entre o sinal transmitido e o sinal de saída decidido é grande para o CMA ao longo das iterações, sendo quase nula para a combinação e o QKLMS supervisionado até mais ou menos a iteração $n=3,5 \times 10^{5}$, em que $k(n) \approx 0,3$, e a partir desse ponto, a quantidade de erros cresce devido ao aumento da degradação do canal.

Figura 39 - Diferença entre o sinal transmitido com atraso $a(n-\Delta)$ e o sinal decidido $\hat{a}(n-\Delta)$, para a combinação do CMA com o QKLMS-DD, como minimização do erro de decisão global ao quadrado $e_{d}^{2}(n)$, dado o Cenário 2-D da Tabela 7 (página 50 ).

CMA: Taxa de Erro $=2,6 \times 10^{-1}$

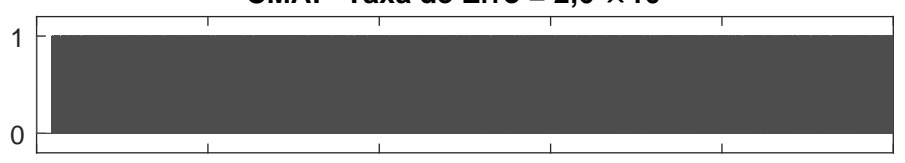

QKLMS supervisionado: Taxa de Erro $=2,8 \times 10^{-3}$

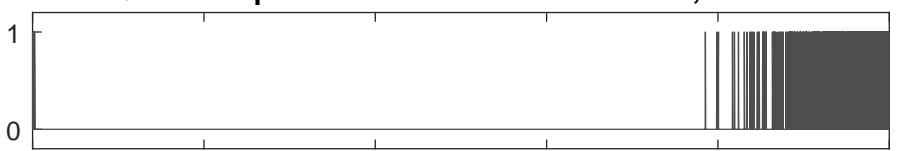

Combinação CMA $\times$ QKLMS-DD: Taxa de Erro = 5,6 $\times 10^{-3}$

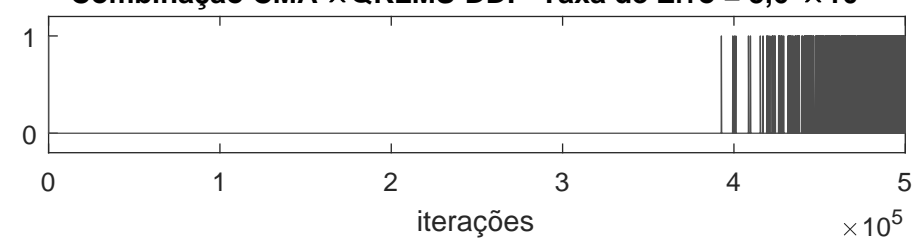

Fonte: Autora.

No Cenário 2-E, o canal é linear e possui um nulo espectral, o que significa que é um canal difícil de equalizar com o LTE. A Figura 40 mostra os resultados para a combinação do CMA com o DFE-NLMS-DD, em que são utilizados $\mu_{1}=10^{-4}, \mu_{2}=\mu_{\mathrm{DFE}}=0,2, \mu_{\alpha}=5,0 \times 10^{-2}$, $\alpha^{+}=4$, e os números de coeficientes dos filtros são $M_{1}=7, M_{2 f}=7$ e $M_{2 b}=5$. Como esperado, o CMA, mesmo com o desempenho ruim, faz o desempenho do DFE-NLMS-DD melhorar e a combinação chaveia para o modo DD. Analisando os resultados da Figura 40 e 41, verifica-se que a combinação funciona bem, uma vez que o seu MSE diminui com as iterações e sua taxa de erro vai para 0. Dessa forma, o desempenho da combinação é próximo ao do DFE-NLMS supervisionado. 
Figura 40 - Exemplo de combinação convexa de um equalizador CMA com um DFE-NLMS-DD para o Cenário 2-E da Tabela 7 (página 50). Em que se usa o erro de decisão global $e_{d}(n), \mu_{1}=10^{-4}$, $\mu_{2}=\mu_{\mathrm{DFE}}=0,2, \mu_{\alpha}=5,0 \times 10^{-2}, M_{1}=7, M_{2 f}=7$ e $M_{2 b}=5$ e $\alpha^{+}=4$. O MSE e E $\{\lambda(n)\}$ são calculados para 100 realizações.
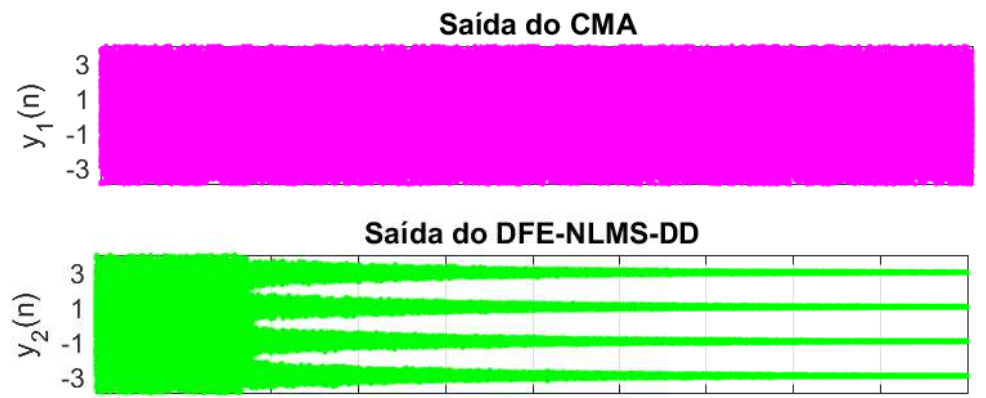

Saída global da combinação

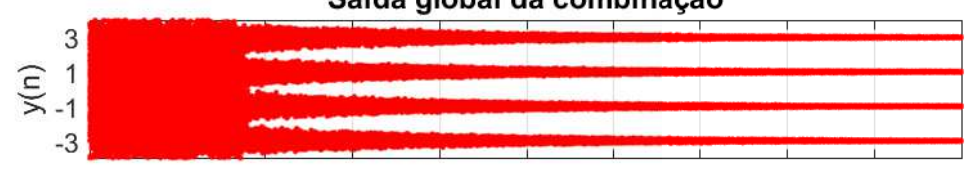

Saída do DFE-NLMS supervisionado
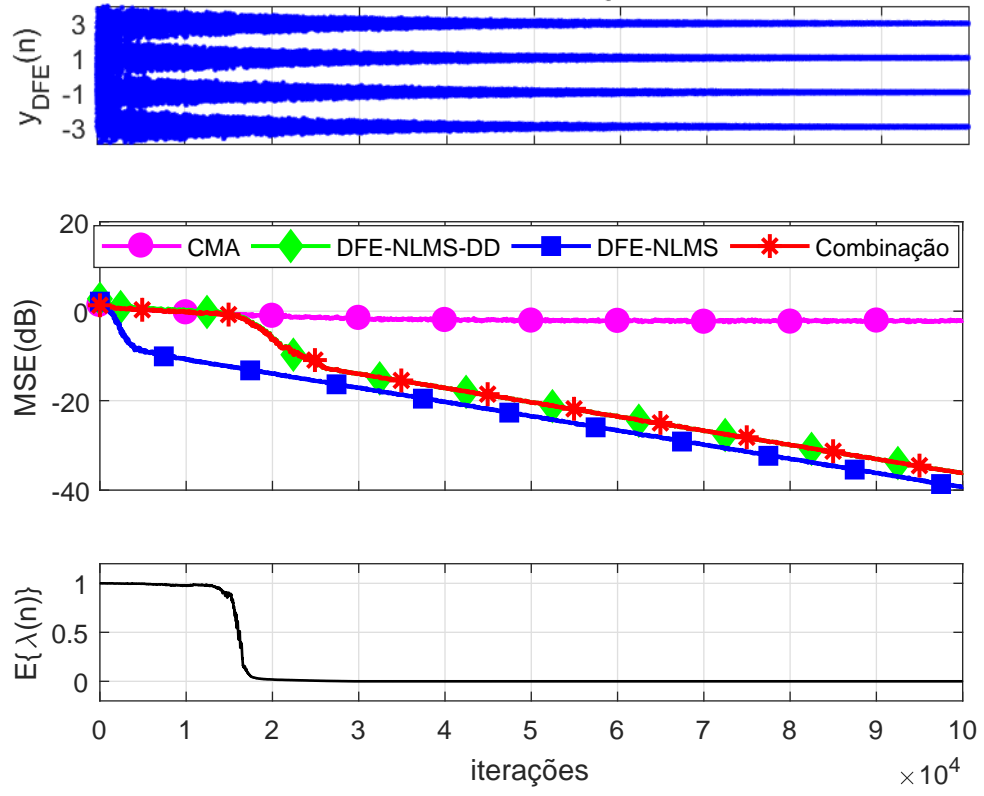

Fonte: Autora. 
Figura 41 - Diferença entre o sinal transmitido com atraso $a(n-\Delta)$ e o sinal decidido $\hat{a}(n-\Delta)$, para a combinação do CMA com o QKLMS-DD, como minimização do erro de decisão global ao quadrado $e_{d}^{2}(n)$, dado o Cenário 2-E da Tabela 7 (página 50 ).

CMA: Taxa de Erro $=2,2 \times 10^{-1}$

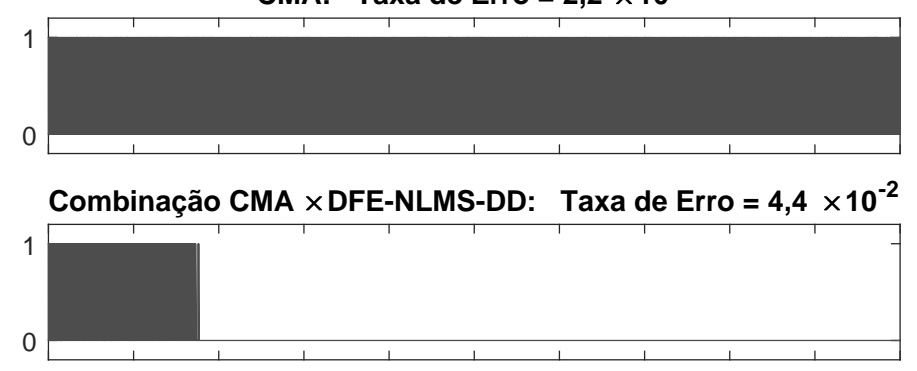

DFE-NLMS supervisionado: Taxa de Erro $=7,3 \times 10^{-3}$

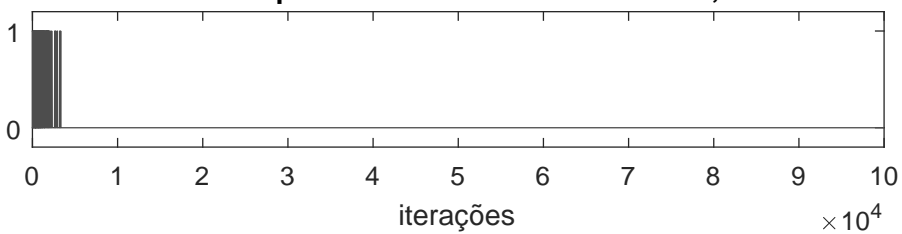

Fonte: Autora.

Apesar do QKLMS ser uma solução adequada à equalização de canais não lineares, a combinação do CMA com o QKLMS-DD foi simulada para o Cenário 2-E para verificar a aplicação dessa solução em um canal linear com nulo espectral. Os resultados são mostrados na Figura 42, em que são utilizados $\mu_{1}=10^{-4}, \mu_{2}=\mu_{\mathrm{QKLMS}}=0,5, \mu_{\alpha}=10^{-1}, \sigma=1, \rho=0,1$ e $\alpha^{+}=4$, sendo os números de coeficientes dos filtros iguais a $M_{1}=5$ e $M_{2}=M_{\mathrm{QKLMS}}=3$.

Os resultados mostram que a combinação chaveou para o QKLMS-DD ao longo das iterações, pois $\mathrm{E}\{\lambda(n)\} \approx 0$. No entanto, apesar das saídas $y_{2}(n)$ e $y(n)$ convergirem para uma solução onde é possível observar os 4 níveis do sinal transmitido, as respectivas curvas de MSE dessas saídas convergiram para o patamar de $7,30 \mathrm{~dB}$, indicando que seus desempenhos foram na realidade ruins. A curva de MSE do QKLMS supervisionado convergiu para 4,91 dB, patamar inferior ao alcançado pela combinação, apesar da saída $y_{Q K L M S}(n)$ aparentar ter sido pior. A Figura 43, com os gráficos da ocorrência de erro nas saídas decididas, mostra que as taxas de erro do CMA, da combinação e do QKLMS supervisionado foram altas, corroborando com o que foi observado nas curvas de MSE. Portanto, acredita-se que a combinação resultou em uma solução degenerada, pois apesar das saídas convergirem para uma solução aparentemente boa, essa solução não tem relação alguma com o sinal transmitido. 
Figura 42 - Exemplo de combinação convexa de um equalizador CMA e com um QKLMS-DD para o Cenário 2-E da Tabela 7 (página 50). Em que se usa o erro de decisão global $e_{d}(n), \mu_{1}=10^{-4}$, $\mu_{2}=\mu_{\mathrm{QKLMS}}=0,5, \mu_{\alpha}=10^{-1}, M_{1}=5, M_{2}=M_{\mathrm{QKLMS}}=3, \sigma=1, \rho=0,1$ e $\alpha^{+}=4 . \mathrm{O} \mathrm{MSE}$ e $\mathrm{E}\{\lambda(n)\}$ são calculados para 100 realizações.
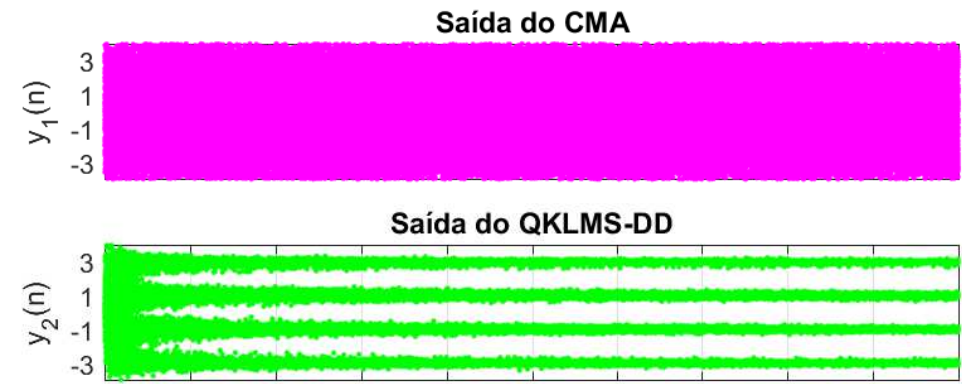

Saída global da combinação

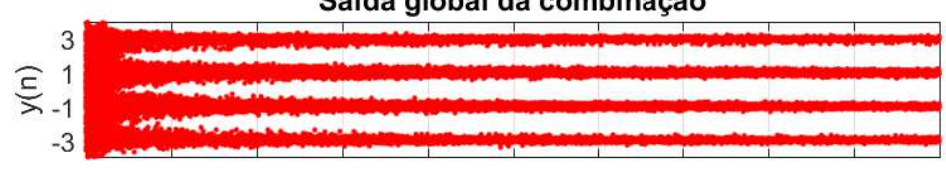

Saída do QKLMS supervisionado
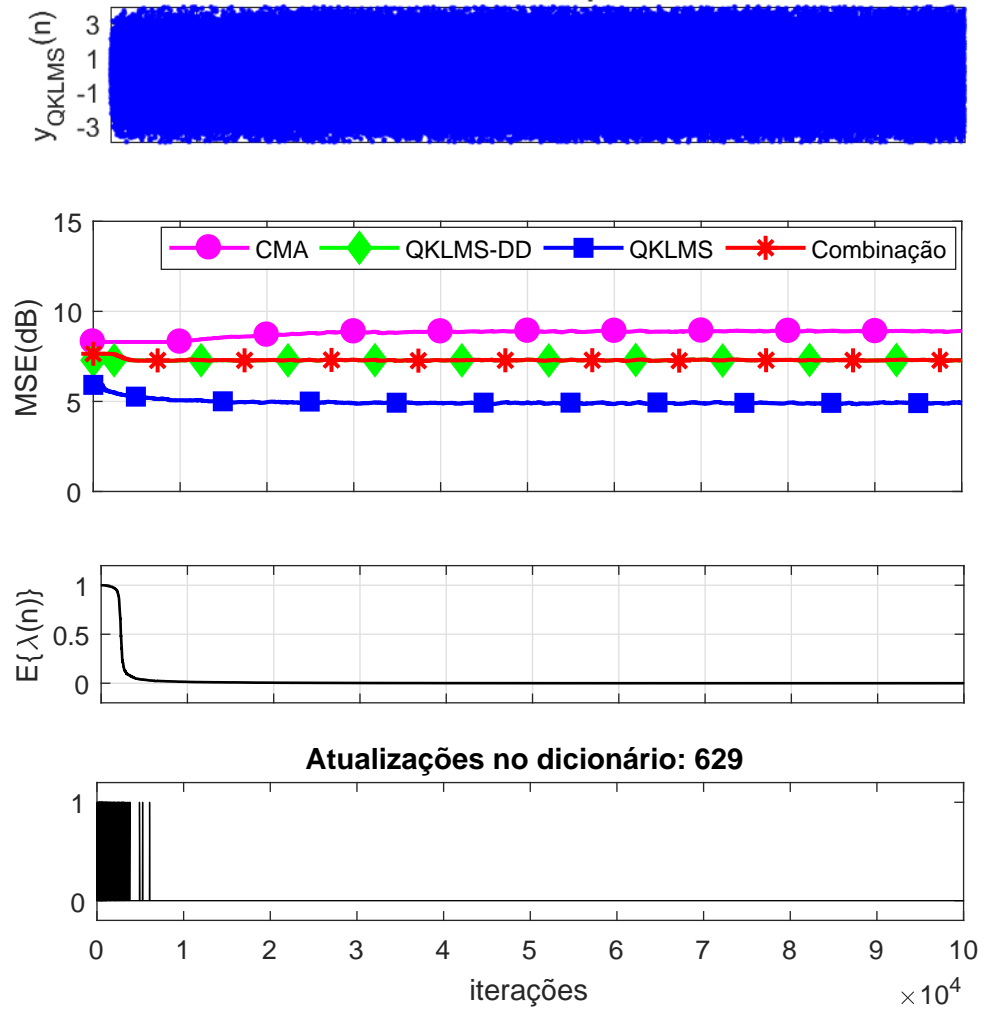

Fonte: Autora. 
Figura 43 - Diferença entre o sinal transmitido com atraso $a(n-\Delta)$ e o sinal decidido $\hat{a}(n-\Delta)$, para a combinação do CMA com o QKLMS-DD, como minimização do erro de decisão global ao quadrado $e_{d}^{2}(n)$, dado o Cenário 2-E da Tabela 7 (página 50).

CMA: Taxa de Erro $=7,2 \times 10^{-1}$

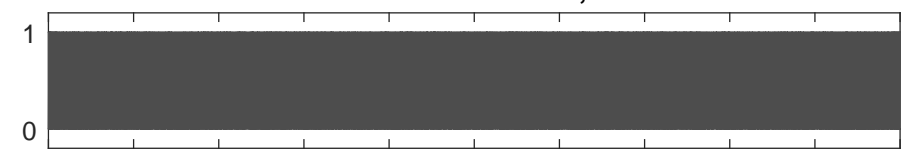

Combinação CMA $\times$ QKLMS-DD: Taxa de Erro $=6,5 \times 10^{-1}$

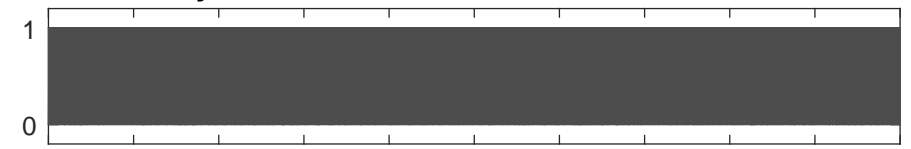

QKLMS supervisionado: Taxa de Erro $=4,8 \times 10^{-1}$

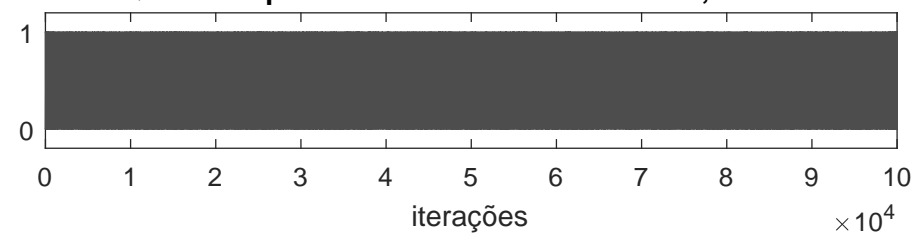

Fonte: Autora.

As soluções degeneradas também podem ocorrer no esquema de combinação do CMA com o DFE-NLMS-DD, devido à utilização da decisão global da combinação como sinal desejado para o DFE. Contudo, essas soluções não foram observadas nas simulações deste capítulo, realizadas com essa combinação. Para evitar tais soluções pode-se utilizar o método proposto em (SZCZECINSKI; GEI, 2002), que possui uma restrição na função custo minimizada pelos filtros direto e de realimentação. No entanto, a partir de simulações, verifica-se que apesar desse método conseguir de fato evitar soluções degeneradas, elas podem comprometer significativamente o desempenho da combinação e por isso esse tema precisa ser mais bem investigado em um trabalho futuro.

Na Figura 44 são mostradas as curvas de SER em função da SNR para o CMA, o DFENLMS e o QKLMS supervisionados, os esquemas de combinação do CMA com o DFE-NLMSDD e do CMA com o QKLMS-DD, levando em conta o Cenário 2-C da Tabela 7 (página 50). A SER é calculada após a convergência de cada algoritmo, comparando o sinal transmitido com a saída decidida e contando o número de erros. Foram desprezados $10^{5}$ símbolos devido à convergência e usados $10^{6}$ símbolos para estimar a SER para cada valor de SNR. Como esperado, a curva com o melhor desempenho é a do QKLMS supervisionado, cuja SER diminui com o aumento da SNR. A curva da combinação do CMA com QKLMS-DD apresentou um 
desempenho muito próximo ao do QKLMS, sendo a SER ligeiramente maior na maioria dos pontos. Embora o DFE-NLMS supervisionado tenha apresentado menos erros que o CMA e a combinação do CMA com o DFE-NLMS-DD, todos esses algoritmos apresentaram um desempenho ruim em termos de SER.

Figura 44 - SER em regime em função da SNR para o CMA, DFE-NLM e QKLMS supervisionado, combinação do CMA com o DFE-NLMS-DD e com o QKLMS-DD, para o Cenário 2-C da Tabela 7 (página 50).

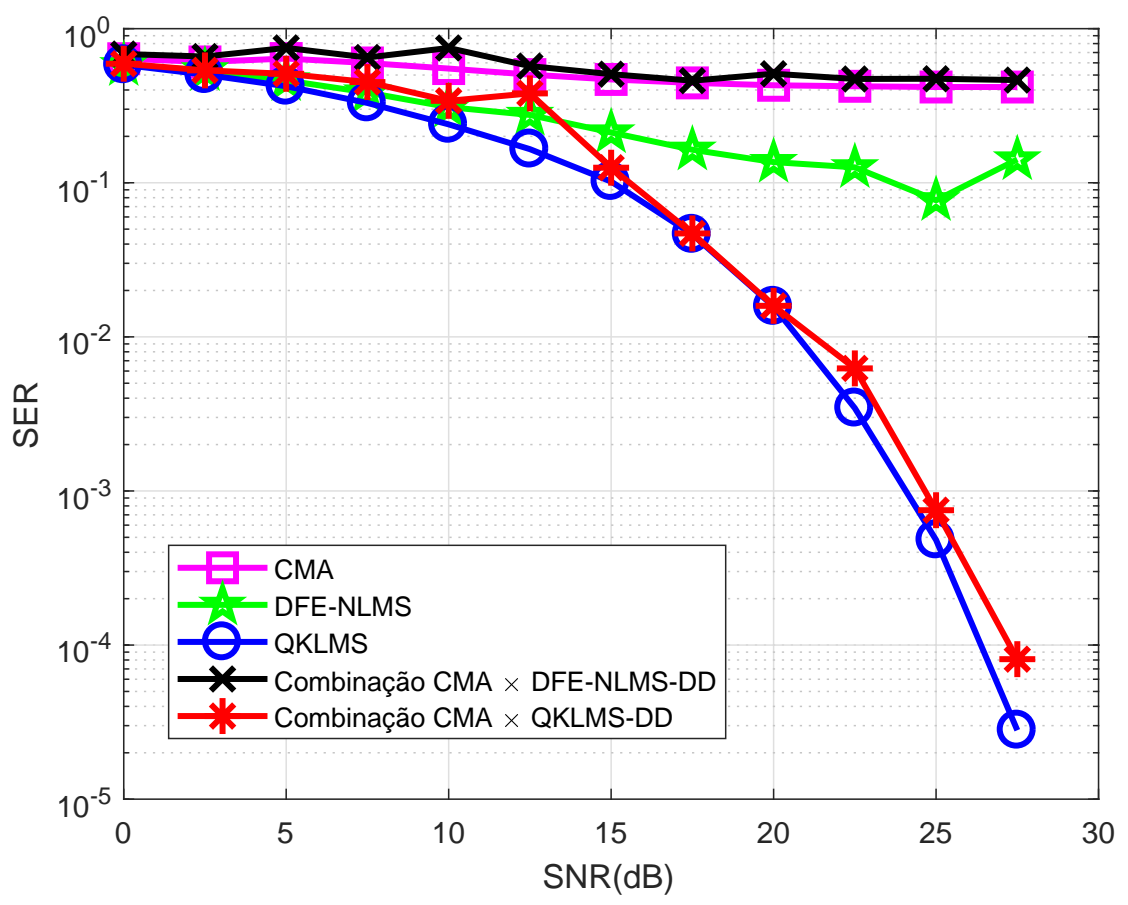

Fonte: Autora.

\subsection{Conclusão}

Neste capítulo, foram propostas soluções adaptativas para a equalização cega de canais baseadas na combinação convexa entre um equalizador cego e um equalizador no modo de decisão direta. Estas soluções foram obtidas inspirando-se na solução existente para a equalização cega que permite o chaveamento automático entre os modos de treinamento cego e o de decisão direta, evitando a seleção a priori de um limiar de MSE (SILVA; ARENAS-GARCÍA, 2013). Com o objetivo de estender essa solução para equalização de canais mais complicados, como os que possuem nulos espectrais e/ou não linearidades, propõem-se a combinação entre o algoritmo CMA e o algoritmo DFE-NLMS-DD e a combinação entre o CMA e o algoritmo QKLMS-DD. Como o DFE e o QKLMS são soluções não lineares, o desempenho desses es- 
quemas foi avaliado por meio de simulações numéricas, que atestaram que mesmo o CMA sendo uma solução mais adequada para canais lineares mais fáceis, a combinação consegue funcionar em alguns casos, sendo a combinação com o DFE mais apropriada para canais com nulos espectrais e a combinação com o QKLMS-DD mais apropriada para canais não lineares. 


\section{RESTAURAÇÃO DE IMAGENS}

Este capítulo aborda a aplicação de equalizadores adaptativos para a restauração cega de imagens, onde o esquema da combinação convexa entre um equalizador cego e um equalizador no modo DD é estendido para um sinal de imagem. Na Seção 4.1, a formulação do problema é apresentada. Na Seção 4.2, são discutidos os procedimentos utilizados para possibilitar o uso dos equalizadores adaptativos na restauração de imagens. Na Seção 4.3, descrevese como exemplo um dos algoritmos de combinação usado. E em seguida, na Seção 4.4, são apresentados os parâmetros de avaliação das simulações, as degradações utilizadas, e os resultados obtidos com a combinação usando soluções lineares e não lineares como equalizador DD. Por fim, a conclusão do capítulo é feita na Seção 4.5 .

\subsection{Formulação do problema}

Na restauração de imagens com filtros adaptativos, o problema é tratado como uma equalização adaptativa, onde a imagem degradada por uma PSF faz o papel de um sinal corrompido por um canal de comunicação e o filtro adaptativo tem a função de reverter os efeitos da PSF, gerando uma estimativa da imagem original. Assim, é possível utilizar algoritmos de equalização cega para a desconvolução cega de imagens, como é o caso do CMA, que foi estendido para duas dimensões e usado na restauração de imagens em (VURAL; SETHARES, 2002). Dessa forma, os esquemas cegos baseados na combinação convexa de equalizadores, discutidos no Capítulo 3, também podem ser estendidos para a restauração cega de imagens, como será detalhado na Seção 4.3. Nesses casos, devido ao uso do CMA, consideram-se os pixels da imagem original mapeados em um sinal PAM, o que pode ser feito incluindo um dispositivo de mapeamento no sistema de aquisição da imagem.

A Figura 45 mostra o esquema geral da desconvolução de uma imagem com o uso de algoritmos de equalização cega, em que se considera a imagem original $\mathbf{F}$, de dimensão $N_{1} \times N_{2}$ e $B$-bits / pixel, mapeada em um sinal $2^{B}$-PAM, modelado pelo alfabeto $\pm 1, \pm 3, \ldots, \pm\left(2^{B}-1\right)$ (VURAL; SETHARES, 2006). Essa imagem sofre os efeitos da PSF e do ruído, resultando na imagem degradada G. Por meio da utilização de uma janela de seleção e um percurso de varredura (definidos a seguir nas subseções 4.2 .1 e 4.2.3, respectivamente), uma matriz 
$\mathbf{U}_{n}$ de dimensão $M \times M$ é definida para cada pixel da imagem e os elementos dessa matriz são rearranjados em um vetor coluna $\mathbf{u}(n)$. Esse vetor é utilizado como entrada de um filtro adaptativo com $M^{2}$ coeficientes, agrupados no vetor coluna $\mathbf{w}(n-1)$ e a saída do filtro é calculada pelo produto interno $y(n)=\mathbf{u}^{T}(n) \mathbf{w}(n-1)$. A saída $y(n)$ entra em um decisor e uma estimativa do pixel $\mathbf{F}\left(k_{1}, k_{2}\right)$, designada por $\widehat{\mathbf{F}}\left(k_{1}, k_{2}\right)$, é obtida. Assim, usando $y(n)$, $\widehat{\mathbf{F}}\left(k_{1}, k_{2}\right)$ e informações estatísticas de $\mathbf{F}$, o algoritmo atualiza o vetor $\mathbf{w}(n-1)$.

Figura 45 - Desconvolução de imagens usando algoritmos de equalização cega.

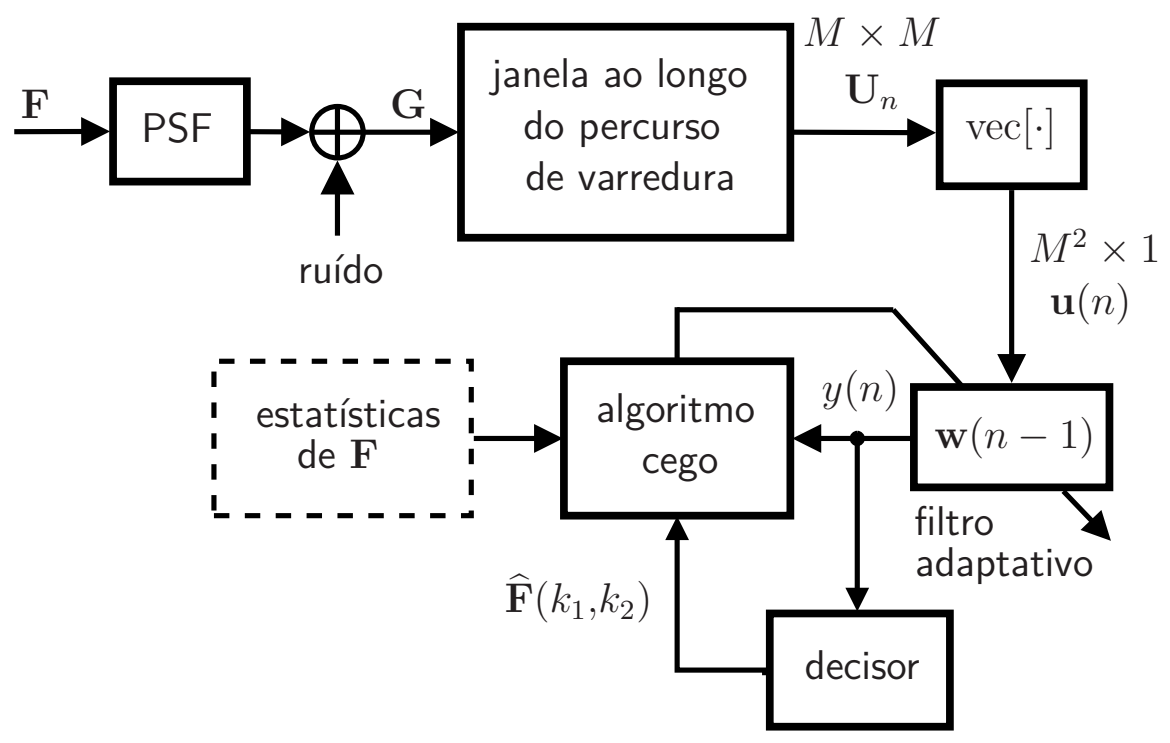

Fonte: Autora.

Em geral, os algoritmos adaptativos precisam de um número de iterações suficiente para atingir o regime estacionário e convergir. Na aplicação em imagens, o número de iterações é igual ao número de pixels da imagem, o que pode ser um valor insuficiente para garantir a convergência do algoritmo. Portanto, a filtragem deve ser realizada repetidas vezes sobre a mesma imagem até que a convergência seja alcançada por todos os pixels, sendo que o número de repetições necessárias depende do nível da degradação causada pela PSF (KUNDUR; HATZINAKOS, 1996a; VURAL; SETHARES, 2006; ABREU; SILVA, 2011; MENDES-FILHO; MIRANDA; SILVA, 2014).

Os procedimentos utilizados para a aplicação dos filtros adaptativos na restauração de imagens são detalhados a seguir. 


\subsection{Procedimentos para o uso de algoritmos adaptativos na restauração de imagens}

O problema da restauração de imagens com filtros adaptativos difere-se da equalização adaptativa principalmente pelo fato da imagem ser um sinal bidimensional e por dispor de menos amostras que um sinal de comunicação normalmente dispõe. Por isso, são necessários os procedimentos descritos a seguir para possibilitar o uso dos filtros adaptativos na restauração de imagens.

\subsubsection{Definição de janelas}

Para a estimação de cada pixel da imagem, é necessário selecionar uma janela $\mathbf{U}_{n}$ que contenha o pixel de interesse, já que os algoritmos de filtragem utilizam a informação das vizinhanças para a estimação do mesmo. A Figura 46 mostra um tipo de janela centralizada, utilizada neste trabalho, no qual um pixel central representado por $\star$ é estimado utilizando os pixels à sua volta (veja por exemplo, (VURAL; SETHARES, 2002) e suas referências). A janela é uma matriz de dimensão $M \times M$, em que $M$ deve ser ímpar. O tamanho de $M$ influencia na velocidade de convergência do algoritmo, uma vez que quanto maior a quantidade de pixels na janela, maior as variações entre o pixel desejado e a sua vizinhança, o que pode dificultar a sua estimação.

Figura 46 - Modelo de seleção de janela (dimensão $M \times M$ e $M$ ímpar) e inicialização de borda.
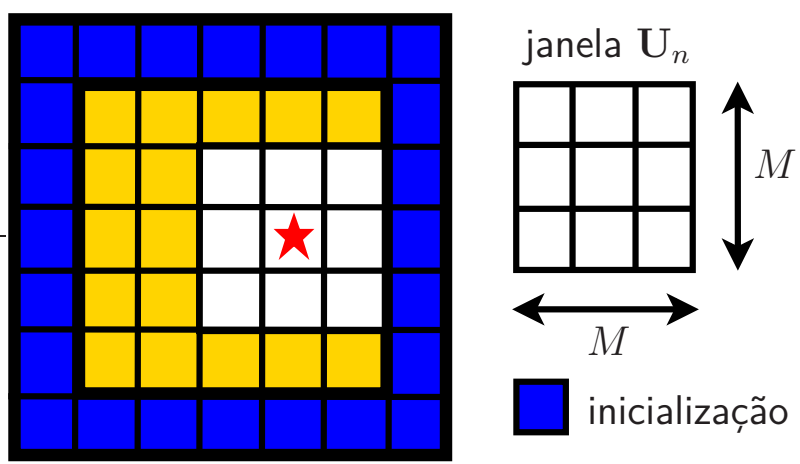

Fonte: Figura 4 de (MENDES-FILHO; MIRANDA; SILVA, 2014)

\subsubsection{Interfaces de borda}

Para estimar um pixel presente na região da borda da imagem, é necessário conhecer os pixels da sua vizinhança, que nesse caso não é bem definida. Por isso, o uso da janela centra- 
lizada requer um tratamento de borda específico ao redor de toda a imagem, representado na Figura 46, podendo ser encarado como uma condição de inicialização dos algoritmos.

Para facilitar o trabalho dos filtros adaptativos, o ideal é evitar mudanças bruscas na imagem. Uma boa solução é o uso de uma interface de borda replicada e reversa, obtida por meio do espelhamento dos pixels mais externos da borda da imagem degradada, como mostrado na Figura 47. Com isso, as informações além da extremidade da imagem tem condições semelhantes às da borda, o que torna as estimativas dos filtros mais efetivas.

Figura 47 - Exemplo de borda replicada e reversa.

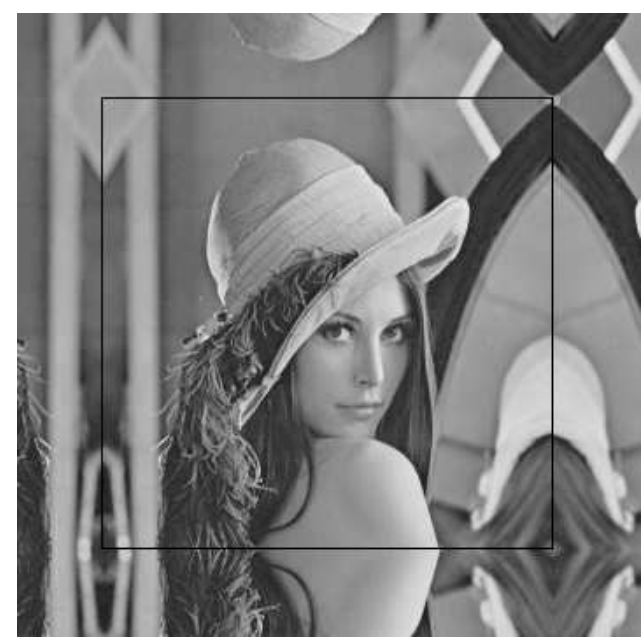

Fonte: Autora.

\subsubsection{Percurso de varredura}

Nos algoritmos adaptativos bidimensionais, o percurso de varredura corresponde ao caminho que a janela $\mathbf{U}_{n}$ faz sobre a imagem para estimar cada pixel. Normalmente, a varredura ocorre linha por linha, sempre da esquerda para direita (HADHOUD; THOMAS, 1998). Um problema desse método é que podem ocorrer mudanças muito bruscas nos coeficientes do filtro quando uma nova linha é percorrida devido à não estacionariedade das imagens reais.

Para resolver esse problema, deve-se evitar variações abruptas entre uma janela e outra. Uma forma de garantir isso, é aplicando uma varredura que muda de direção a cada nova linha varrida, como representado na Figura 48. Assim as condições estatísticas de cada região são preservadas e os transientes vistos na varredura convencional são minimizados (ABREU, 2011). Além disso, pode-se utilizar um esquema de sucessivas varreduras alternadas nas direções horizontal e vertical, conforme mostra a Figura 48, em que inicialmente é feita uma varredura horizontal completa na imagem, seguida por uma varredura vertical, uma outra 
horizontal e uma última vertical, considerando que cada nova varredura se inicia a partir do pixel da última iteração da varredura anterior. Esse conjunto de quatro varreduras alternadas é denominado ciclo de varredura do algoritmo, que é repetido até se obter a convergência do filtro (MENDES-FILHO; MIRANDA; SILVA, 2014).

Figura 48 - Combinação dos percursos de varreduras horizontais e verticais.

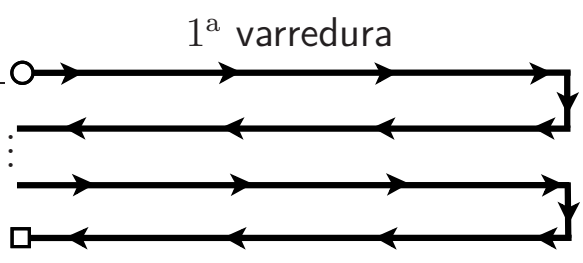

$3^{\mathrm{a}}$ varredura

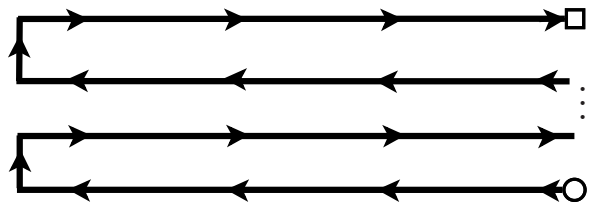

Fonte: Figura adaptada de (MENDES-FILHO; MIRANDA; SILVA, 2014).

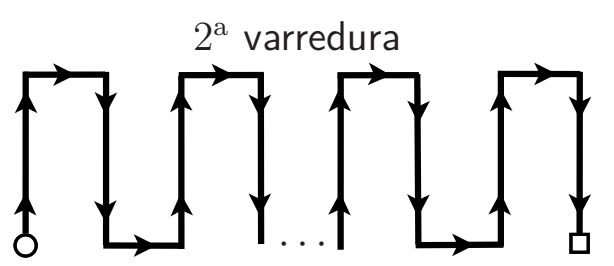

$4^{\mathrm{a}}$ varredura

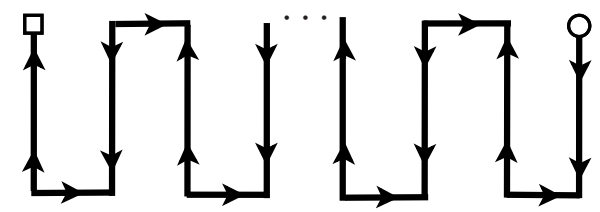

\subsubsection{Imagem como sinal unidimensional}

Os procedimentos definidos anteriormente foram desenvolvidos considerando a matriz $\mathbf{U}_{n}$ como sinal de entrada do filtro, levando o algoritmo adaptativo a trabalhar com operações matriciais. Para evitar essas operações e facilitar a extensão dos algoritmos de equalização para restauração de imagens, optou-se neste trabalho por utilizar a janela $\mathbf{U}_{n}$ na forma de um vetor. Assim, os elementos da matriz $\mathbf{U}_{n}$ são rearranjados em um vetor coluna $\mathbf{u}(n)$ por meio de um operador de vetorização, denotado por vec[·], conforme é mostrado no exemplo a seguir para uma matriz $\mathbf{A}$, de dimensão $3 \times 3$, dada por

$$
\mathbf{A}=\left(a_{i j}\right)_{3 \times 3}=\left(\begin{array}{ccc}
a_{11} & a_{12} & a_{13} \\
a_{21} & a_{22} & a_{23} \\
a_{31} & a_{32} & a_{33}
\end{array}\right)
$$

em que a vetorização concatena as linhas da matriz resultando no vetor coluna

$$
\operatorname{vec}[\mathbf{A}]=\left[\begin{array}{lllllllll}
a_{11} & a_{12} & a_{13} & a_{21} & a_{22} & a_{23} & a_{31} & a_{32} & a_{33}
\end{array}\right]^{T} .
$$

Portanto, os algoritmos de filtragem adaptativa podem ser estendidos diretamente para a reconstrução de imagens, desde que os conceitos discutidos nesta seção sejam atendidos. 


\subsection{Restauração cega de imagens com a combinação de equalizadores adaptativos}

Adotando os procedimentos discutidos na Seção 4.2, a combinação convexa entre um equalizador cego e um equalizador no modo DD é estendida para a desconvolução cega de imagens, representada pelo bloco "algoritmo cego" no esquema da Figura 45 (página 75). O vetor $\mathbf{u}(n)$, gerado a partir da vetorização de uma janela $\mathbf{U}_{n}$, é o vetor de entrada da combinação, cuja implementação é similar às dos algoritmos discutidos no Capítulo 3, substituindo o sinal decidido $\hat{a}(n)$ pela estimativa do pixel decidido $\widehat{\mathbf{F}}\left(k_{1}, k_{2}\right)$ e o fator de dispersão $r$ por

$$
r=\frac{\mathrm{E}\left\{\mathbf{F}^{4}\left(k_{1}, k_{2}\right)\right\}}{\mathrm{E}\left\{\mathbf{F}^{2}\left(k_{1}, k_{2}\right)\right\}}
$$

que contem as informações estatísticas da imagem original $\mathbf{F}$.

O algoritmo da combinação entre o CMA e o NLMS-DD com o erro de decisão global $e_{d}(n)$ está resumido na Tabela 8, para cada ciclo de varredura. Assim, como um ciclo contém quatro varreduras alternadas, uma imagem de dimensão $N_{1} \times N_{2}$ possui $N=4 \times N_{1} \times N_{2}$ iterações/ciclo. Cabe ressaltar que o erro $e_{d}(n)$ na equação de atualização da variável auxiliar $\alpha(n)$ pode ser substituído pelo erro do CMA $\varepsilon(n)$, conforme foi discutido na Seção 3.1. Os esquemas de combinação levando em conta soluções não lineares, discutidos na Seção 3.2, podem ser estendidos para imagens, seguindo os mesmos procedimentos do algoritmo da Tabela 8.

Tabela 8: Sumário do algoritmo de combinação convexa entre o CMA e o NLMS-DD aplicado à restauração de imagens, com minimização do erro de decisão global ao quadrado $e_{d}^{2}(n)$.

Combinação convexa entre o CMA e o NLMS-DD aplicada à restauração de imagens

Escolha dos passos de adaptação $\mu_{1}, \mu_{2}>0 ; \quad r=\frac{\mathrm{E}\left\{\mathbf{F}^{4}(n)\right\}}{\mathrm{E}\left\{\mathbf{F}^{2}(n)\right\}}$

Inicialização

Definição de janela $\mathbf{U}_{n}$

Definição de condição de borda

Definição de varredura

Continuação na próxima página 
Combinação convexa entre o CMA e o NLMS-DD aplicada à restauração de imagens

$\mathbf{w}_{1}(0)=\left[\begin{array}{lllllll}0 & \cdots & 0 & 1 & 0 & \cdots & 0\end{array}\right]^{T} ; \quad \mathbf{w}_{2}(0)=\mathbf{0}_{M^{2}}$

$\alpha(0)=\alpha^{+} ; \quad p(0)=1 ; \quad \eta=0,9$

\section{Cálculos}

Para $n=1,2, \cdots, N$ (isto é, para cada posição $\left(k_{1}, k_{2}\right)$, em que $k_{1}=1,2, \cdots, N_{1}$ e $\left.k_{2}=1,2, \cdots, N_{2}\right)$, faça:

$\mathbf{u}(n)=\operatorname{vec}\left[\mathbf{U}_{n}\right]$

\% Cálculo das saídas:

$y_{1}(n)=\mathbf{u}^{T}(n) \mathbf{w}_{1}(n-1)$

$y_{2}(n)=\mathbf{u}^{T}(n) \mathbf{w}_{2}(n-1)$

Se: $|\alpha(n-1)|>\alpha^{+}: \alpha(n-1) \leftarrow \alpha^{+} \operatorname{sign}[\alpha(n-1)]$

$\lambda(n)=\varphi[\alpha(n-1)]=\frac{\operatorname{sgm}[\alpha(n-1)]-\operatorname{sgm}\left[-\alpha^{+}\right]}{\operatorname{sgm}\left[\alpha^{+}\right]-\operatorname{sgm}\left[-\alpha^{+}\right]}$

$y(n)=\lambda(n) y_{1}(n)+[1-\lambda(n)] y_{2}(n)$

\% Cálculo dos erros:

$\varepsilon_{1}(n)=\left[r-y_{1}^{2}(n)\right] y_{1}(n)$

$\widehat{\mathbf{F}}\left(k_{1}, k_{2}\right)=\operatorname{decisor}[y(n)]$

$e_{d 2}(n)=\widehat{\mathbf{F}}\left(k_{1}, k_{2}\right)-y_{2}(n)$

$e_{d}(n)=\widehat{\mathbf{F}}\left(k_{1}, k_{2}\right)-y(n)$

\% Atualizações:

$\mathbf{w}_{1}(n)=\mathbf{w}_{1}(n-1)+\mu_{1} \varepsilon_{1}(n) \mathbf{u}(n)$

$\mathbf{w}_{2}(n)=\mathbf{w}_{2}(n-1)+\frac{\mu_{2}}{\delta+\|\mathbf{u}(n)\|^{2}} e_{d 2}(n) \mathbf{u}(n)$

$\varphi^{\prime}[\alpha(n-1)]=\frac{\operatorname{sgm}[\alpha(n-1)]\{1-\operatorname{sgm}[\alpha(n-1)]\}}{\operatorname{sgm}\left[\alpha^{+}\right]-\operatorname{sgm}\left[-\alpha^{+}\right]}$

$p(n)=\eta p(n-1)+(1-\eta)\left[y_{1}(n)-y_{2}(n)\right]^{2}$

$\alpha(n)=\alpha(n-1)+\frac{\mu_{\alpha}}{p(n)} e_{d}(n)\left[y_{1}(n)-y_{2}(n)\right] \varphi^{\prime}[\alpha(n-1)]$

Fim

Fonte: Autora. 


\subsection{Resultados de simulações}

Nesta seção são apresentados os parâmetros de avaliação dos resultados, as PSFs utilizadas, e os resultados de simulações obtidos com combinação do CMA com NLMS-DD e com a combinação do CMA com o DFE-NLMS-DD.

\subsubsection{Parâmetros de avaliação}

Para avaliar a qualidade das imagens restauradas, foram utilizadas duas métricas: o erro quadrático médio percentual (\%MSE) (KUNDUR; HATZINAKOS, 1996a, Eq. (25)) e a similaridade estrutural média (mean structural similarity - MSSIM) (WANG et al., 2004, Eq. (17)).

O \%MSE é o erro quadrático médio percentual, obtido por meio da comparação entre a imagem restaurada e a original, sendo calculado por (KUNDUR; HATZINAKOS, 1996a)

$$
\% \mathrm{MSE}=100 \frac{\sum_{\forall\left(k_{1}, k_{2}\right)}\left[\theta \widehat{\mathbf{F}}\left(k_{1}, k_{2}\right)-\mathbf{F}\left(k_{1}, k_{2}\right)\right]^{2}}{\sum_{\forall\left(k_{1}, k_{2}\right)} \widehat{\mathbf{F}}^{2}\left(k_{1}, k_{2}\right)},
$$

em que $\theta$ é um fator de normalização, dado por

$$
\theta=\frac{\sum_{\forall\left(k_{1}, k_{2}\right)} \mathbf{F}\left(k_{1}, k_{2}\right) \widehat{\mathbf{F}}\left(k_{1}, k_{2}\right)}{\sum_{\forall\left(k_{1}, k_{2}\right)} \widehat{\mathbf{F}}^{2}\left(k_{1}, k_{2}\right)} .
$$

O MSSIM, por sua vez, mede a similaridade entre duas imagens e funciona como uma medida de qualidade de uma imagem em relação a outra, assumindo valor máximo igual a um quando as duas imagens são iguais (WANG et al., 2004). Enquanto o \%MSE é uma abordagem que estima erros absolutos, o MSSIM é baseado em características da percepção humana, como sensibilidade ao contraste, percepção de luz e luminância, noção de estrutura e dispersão de ponto (WANG et al., 2004). No cálculo do MSSIM, foi usado o código em MATLAB disponível em https://ece.uwaterloo.ca/ z70wang/research/ssim/, sendo $K=\left[\begin{array}{ll}0.01 & 0.03\end{array}\right]$ e window = fspecial('gaussian', 11, 1.5), parâmetros de entrada da função que calcula o MSSIM.

Como o número de iterações dos algoritmos é muito grande devido às repetidas varreduras sobre a imagem, optou-se por mostrar o valor médio do parâmetro de mistura $\bar{\lambda}$ em cada ciclo de varredura $c$, definido por

$$
\bar{\lambda}(c)=\frac{1}{N} \sum_{n=1}^{N} \lambda(n)
$$


em que $N$ é a quantidade de iterações existentes em um ciclo de varredura.

\subsubsection{PSFs utilizadas}

As degradações utilizadas nas simulações desta seção são definidas na Tabela 9, onde são descritos os modelos das PSFs e os parâmetros utilizados. Cabe destacar, que esses modelos resultam em PSFs simétricas de dimensão $L \times L$. A PSF $\mathbf{H}_{3}\left(k_{1}, k_{2}\right)$ é conhecida como blur gaussiano, sendo $K$ uma constante de normalização para preservar o valor médio da imagem original e $\sigma_{b}^{2}$ a variância do blur, responsável pela severidade da degradação (CAMPISI; EGIAZARIAN, 2007). Essa PSF pode ser gerada pela função do MATLAB dada por fspecial('gaussian', $\left.L, \sigma_{b}\right)$, sendo $\sigma_{b}$ o desvio padrão referente à variância $\sigma_{b}^{2}$. A PSF $\mathbf{H}_{4}\left(k_{1}, k_{2}\right)$ corresponde ao blur de desfocagem uniforme, degradação que ocorre geralmente em imagens de câmeras fotográficas, podendo estar relacionada à distância entre a câmera e o objeto ou aos efeitos de difração da luz (CAMPISI; EGIAZARIAN, 2007). O modelo possui um único parâmetro $R$, denominado raio de desfocagem, e a PSF pode ser obtida por meio da função do MATLAB fspecial('disk', $R$ ), cuja dimensão resultante é $L=2 R+1$. A PSF $\mathbf{H}_{5}\left(k_{1}, k_{2}\right)$ tem o modelo definido em (VURAL; SETHARES, 2006), onde o parâmetro $\beta$ tem relação com a severidade da PSF e $K$ é a constante de normalização.

A partir de simulações com diferentes modelos de degradação, verificou-se que existem PSFs mais fáceis para um filtro adaptativo, e consequentemente a combinação, obter uma boa estimativa de $\mathbf{F}$. Assim, dada uma PSF $\mathbf{H}$ de dimensão $L \times L$, em que $L$ é ímpar, considera-se essa PSF "fácil" se $\mathbf{H}$ apresentar mais energia no seu centro do que nas demais posições, sendo que essa energia decresce com a distância dessas posições em relação ao centro. Tais características são observadas em $\mathbf{H}_{3}, \mathbf{H}_{4}$ e $\mathbf{H}_{5}$, que deram origem aos canais de comunicação $H_{3}, H_{4}$ e $H_{5}$, respectivamente, utilizados nas simulações do Capítulo 3 (descritos na Tabela 4 da página 37). Esses canais também são considerados fáceis, pois suas respostas ao pulso unitário têm mais energia concentrada no termo central, de forma que os efeitos da ISI não são tão fortes e o LTE é suficiente para equalizar. 
Tabela 9: Descrição das degradações de imagem utilizadas nas simulações para a restauração de imagens.

\begin{tabular}{|c|c|}
\hline Modelo de degradação da PSF & Parâmetros utilizados \\
\hline $\mathrm{H}_{3}\left(k_{1}, k_{2}\right)=\exp \left(-\frac{k_{1}^{2}+k_{2}^{2}}{2 \sigma_{b}^{2}}\right)$ & $L=5$ \\
\hline $\begin{array}{c}\mathbf{H}_{3}\left(k_{1}, k_{2}\right)=K \times \mathrm{H}_{3}\left(k_{1}, k_{2}\right) \\
\text { para }\left|k_{1}\right|,\left|k_{2}\right| \leq \frac{L-1}{2}\end{array}$ & $\begin{array}{c}\sigma_{b}^{2}=0,64 \\
K=1 / \sum_{k_{1}} \sum_{k_{2}} \mathrm{H}_{3}\left(k_{1}, k_{2}\right)\end{array}$ \\
\hline $\mathbf{H}_{4}\left(k_{1}, k_{2}\right)= \begin{cases}\frac{1}{\pi R^{2}}, & \text { se } \sqrt{k_{1}^{2}+k_{2}^{2}} \leq R \\
0, & \text { caso contrário }\end{cases}$ & $\begin{array}{c}R=1 \\
L=2 R+1\end{array}$ \\
\hline $\mathrm{H}_{5}\left(k_{1}, k_{2}\right)=\frac{1}{\left(\beta^{2}+k_{1}^{2}+k_{2}^{2}\right)^{3 / 2}}$ & $L=9$ \\
\hline $\begin{array}{c}\mathbf{H}_{5}\left(k_{1}, k_{2}\right)=K \times \mathrm{H}_{5}\left(k_{1}, k_{2}\right) \\
\text { para }\left|k_{1}\right|,\left|k_{2}\right| \leq \frac{L-1}{2}\end{array}$ & $\begin{array}{c}\beta=1,5 \\
K=1 / \sum_{k_{1}} \sum_{k_{2}} \mathrm{H}_{5}\left(k_{1}, k_{2}\right)\end{array}$ \\
\hline
\end{tabular}

Fonte: Autora.

\subsubsection{Resultados da combinação do CMA com o NLMS-DD}

O esquema da combinação do CMA com o NLMS-DD para a restauração de imagens é comparado com os algoritmos NLMS, CMA, e RMA (MENDES-FILHO; MIRANDA; SILVA, 2014), levando em conta as questões de implementação discutidas na Seção 4.2. O algoritmo NLMS é tomado como referência por ser um método supervisionado, pois utiliza a imagem original como sinal desejado. O RMA (Apêndice A) é uma solução para equalização cega mais eficiente que o CMA quando usado em sinais de módulo não constante.

Nas simulações apresentadas a seguir, a imagem original $\mathbf{F}$ passou pela equalização de histograma antes de sofrer a degradação H. Além disso, escolheu-se a combinação adaptada com o erro de decisão global $e_{d}(n)$, pois esse esquema apresentou melhores resultados que o erro do $\mathrm{CMA} \varepsilon(n)$, como será demonstrado mais à frente nesta seção.

Na primeira simulação, considerou-se a imagem Cameraman de dimensão $128 \times 128$ e 
4-bits/ pixel, mostrada na Figura 49(a). Essa imagem foi degradada pela PSF $\mathbf{H}_{3}$ da Tabela 9, resultando na imagem mostrada na Figura 49(b). As imagens restauradas pelos algoritmos NLMS, CMA, RMA e pela combinação depois de $10^{4}$ ciclos são mostradas respectivamente nas Figuras 49(c), 49(d), 49(e) e 49(f). A imagem degradada apresenta \%MSE de 4,874, sendo que o \%MSE atingido pela combinação ao final dos $10^{4}$ ciclos de varredura foi igual a 0,402 , valor inferior ao do CMA $(5,175)$ e ao do RMA $(0,763)$, e mais próximo do NLMS $(0,338)$. Ao comparar as imagens restauradas por cada algoritmo com a imagem original (Figura 49(a)), verifica-se que em termos qualitativos o resultado do CMA foi inferior aos dos demais algoritmos, que apresentaram resultados muito semelhantes à imagem original.

O MSSIM, mostrado na Figura 50 ao longo dos $10^{4}$ ciclos de varredura, corrobora com essa observação. O MSSIM da imagem degradada é igual a 0,766. Ao final dos ciclos, os valores alcançados pelos algoritmos foram: 0,992 (NLMS), 0,977 (RMA), 0,871 (CMA) e 0.990 (combinação). Dessa forma, a combinação foi a que mais se aproximou do algoritmo NLMS, graças ao chaveamento automático para o modo de decisão direta, o que pode ser verificado observando o parâmetro de mistura médio $\bar{\lambda}(c)$, mostrado na Figura 50 , que após alguns ciclos iniciais converge para próximo de zero.

Na segunda simulação, considerou-se a imagem Peppers de dimensão $128 \times 128$ e 5bits/pixel, mostrada na Figura 51(a). Essa imagem foi degradada pela PSF $\mathbf{H}_{4}$ da Tabela 9, resultando na imagem mostrada na Figura 51(b). Os resultados das restaurações com o NLMS, CMA, RMA e a combinação após $10^{4}$ ciclos de varredura são mostrados nas Figuras 51(c), 51(d), 51(e) e 51(f), respectivamente. O \%MSE da imagem degradada é 3,227 e o da imagem resultante da combinação foi 0,451 , valor próximo ao do $\operatorname{NLMS}(0,364)$ e inferior a o do CMA $(3,481)$ e RMA $(2,428)$.

O MSSIM da imagem degradada é 0,906. A Figura 52 mostra as curvas com o MSSIM de cada algoritmo ao longo dos $10^{4}$ ciclos de varredura, onde nota-se que quando o número de ciclos $c$ é aproximadamente $4,0 \times 10^{3}$, a curva da combinação converge para o patamar de 0,987 chegando muito próximo ao do $\operatorname{NLMS}(0,988)$, com $\bar{\lambda}(c) \approx 0$. A curva do CMA converge para 0,890 e a do RMA para 0,923. Assim, verifica-se que a combinação conseguiu restaurar a imagem com qualidade superior a do CMA e RMA e próxima a do NLMS. 
Figura 49 - Efeitos da restauração de imagens com filtros adaptativos. Imagem Cameraman $128 \times 128$ pixels, 4-bits/pixel, degradada pela PSF $\mathbf{H}_{3}$ da Tabela 9 (página 83).

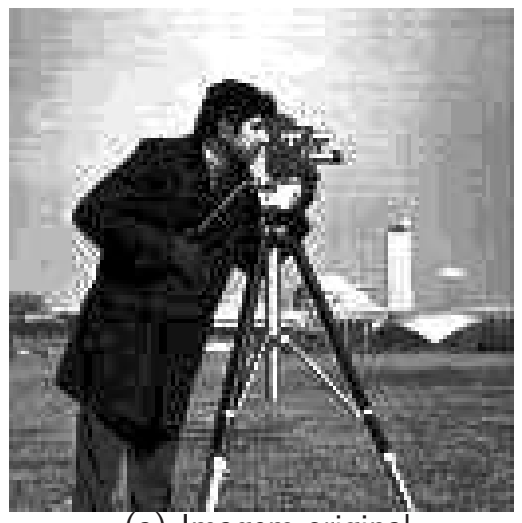

(a) Imagem original

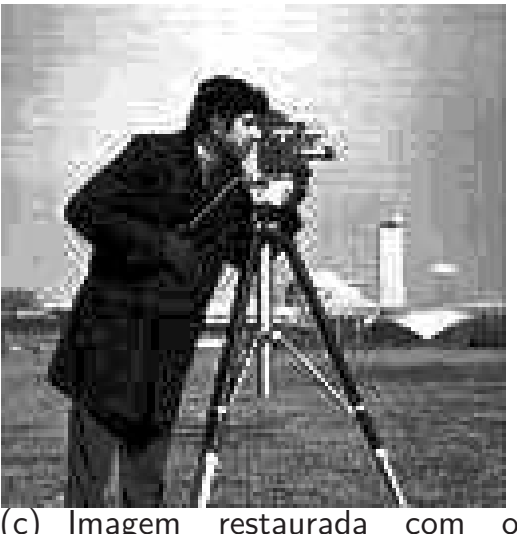

(c) Imagem restaurada NLMS, $\left(\% \mathrm{MSE}=0,338, \mu=10^{-2}\right.$, $M=5)$

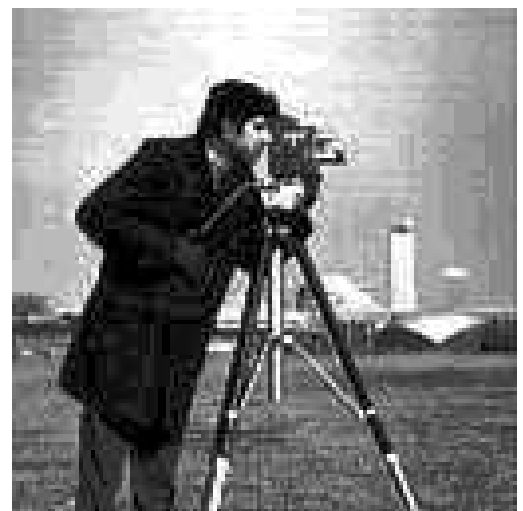

(e) Imagem restaurada com o RMA,

$\left(\% \mathrm{MSE}=0,763, \mu=10^{-4}, M=5\right)$

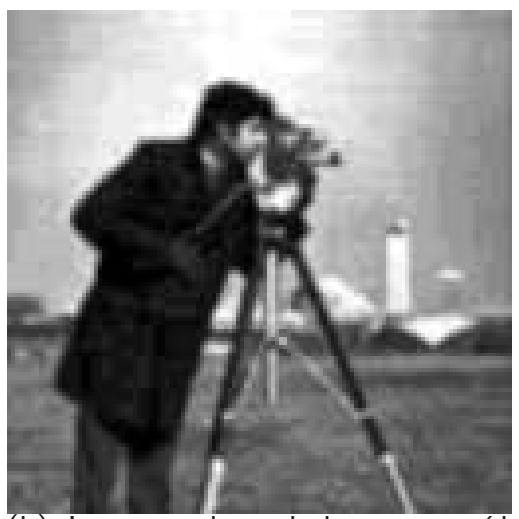

(b) Imagem degradada, sem ruído $(\% \mathrm{MSE}=4,874)$

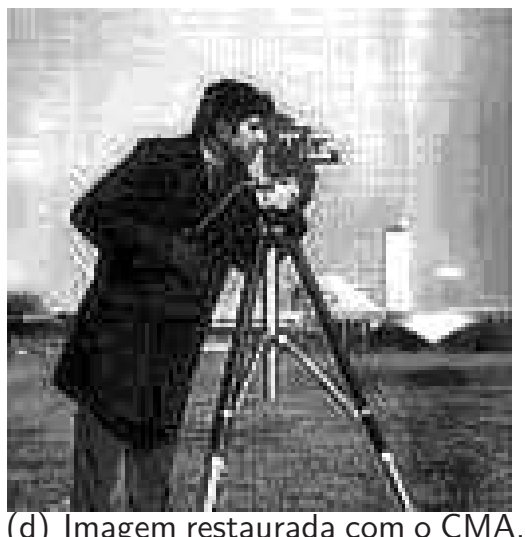

d) Imagem restaurada com o CMA $\left(\% \mathrm{MSE}=5,175, \mu=10^{-8}, M=5\right)$

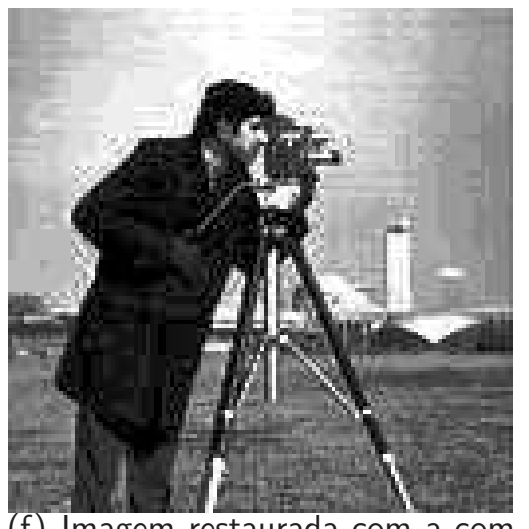

(f) Imagem restaurada com a combinação, $\left(\% \mathrm{MSE}=0,402, \quad \mu_{1}=\right.$ $10^{-8}, \mu_{2}=10^{-4}, \mu_{\alpha}=10^{-4}, M_{1}=$ $\left.M_{2}=5\right)$

Fonte: Autora. 
Figura 50 - Similaridade estrutural média (MSSIM) de cada algoritmo e valor médio do parâmetro de mistura $\bar{\lambda}(c)$ ao longo dos ciclos de varredura para restauração da imagem Cameraman 4-bits/ pixel, degradada pela PSF $\mathbf{H}_{3}$ da Tabela 9 (página 83).
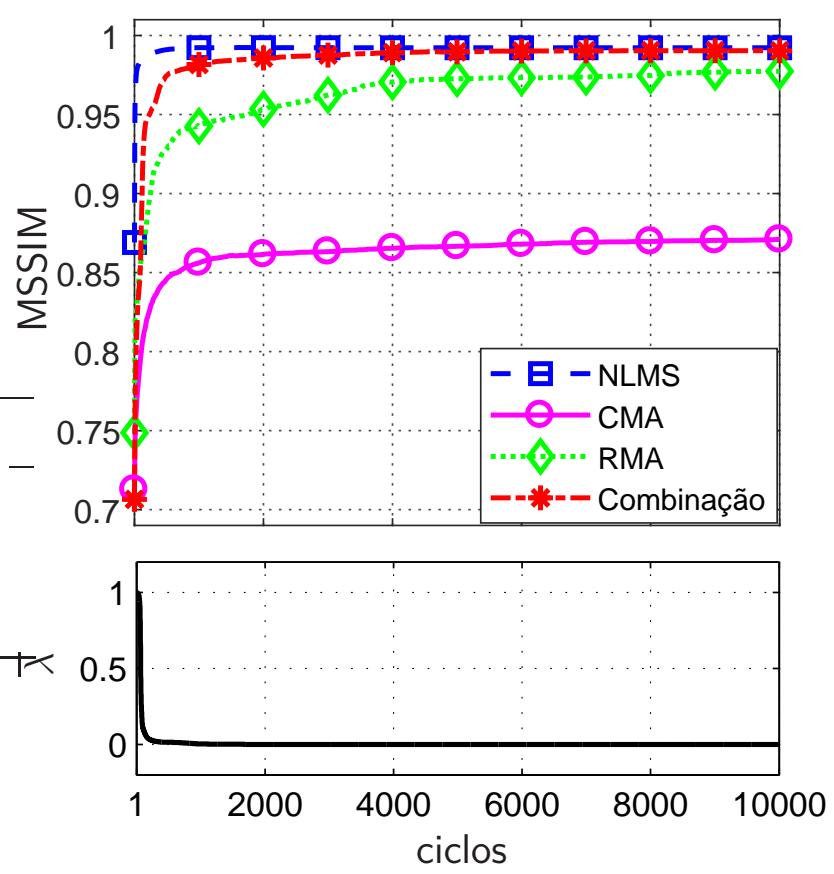

Fonte: Autora.

Na terceira simulação, considerou-se a imagem Lenna de dimensão $128 \times 128$ e 5-bits/ pixel, mostrada na Figura 53(a). Essa imagem foi degradada pela PSF $\mathbf{H}_{5}$ da Tabela 9, resultando na imagem da Figura 53(b). As imagens restauradas pelos algoritmos NLMS, CMA, RMA e pela combinação depois de $10^{4}$ ciclos de varredura são mostradas respectivamente nas Figuras 53(c), 53(d), 53(e) e 53(f). Nesse caso, o \%MSE da imagem degradada era de 9,695, e o \%MSE resultante da combinação $(0,277)$ ficou próximo ao do $\operatorname{NLMS}(0,180)$, sendo ambos inferiores ao do RMA $(4,993)$ e ao do CMA $(6,520)$. 
Figura 51 - Efeitos da restauração de imagens com filtros adaptativos. Imagem Peppers $128 \times 128$ pixels, 5-bits/pixel, degradada pela PSF $\mathbf{H}_{4}$ da Tabela 9 (página 83).

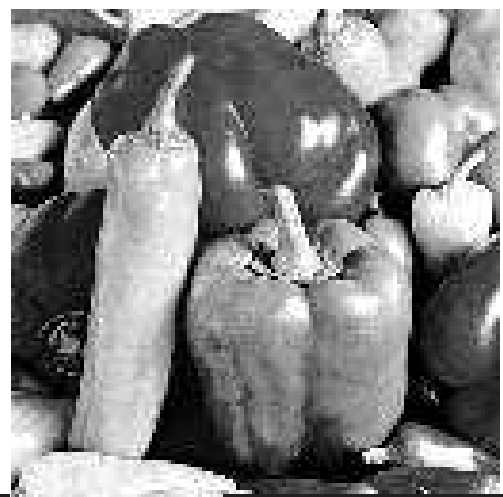

(a) Imagem original $(128 \times 128,5-$ bits/pixel)

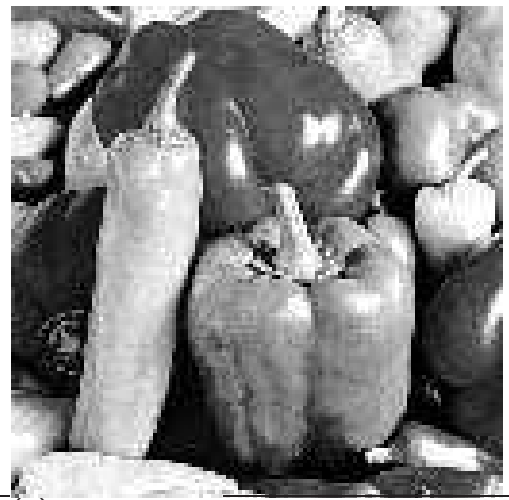

(c) Imagem restaurada com o NLMS

$\left(\% \mathrm{MSE}=0,364, \mu=5,0 \times 10^{-2}\right.$, $M=9)$

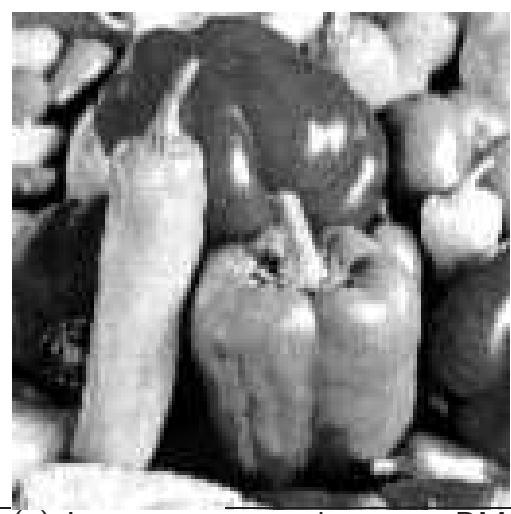

(e) Imagem restaurada com o RMA $\left(\% \mathrm{MSE}=2,428, \mu=4,0 \times 10^{-4}\right.$, $M=5)$

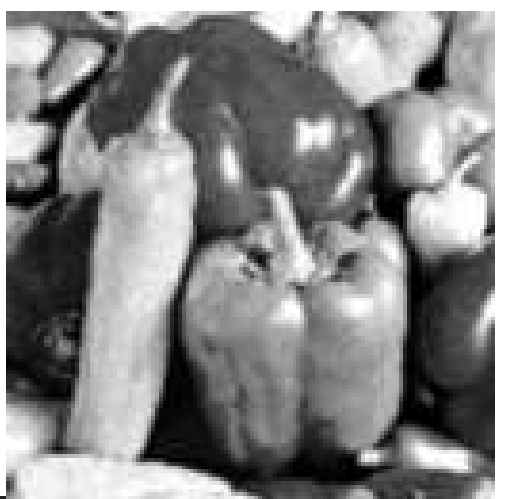

(b) Imagem degradada, sem ruído $(\%$ MSE $=3,227)$

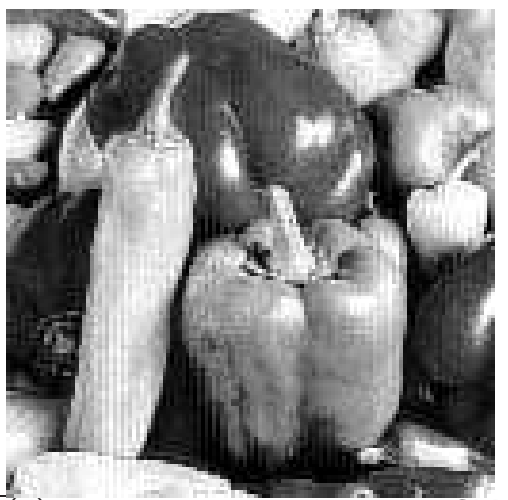

(d) Imagem restaurada com o CMA $\left(\% \mathrm{MSE}=3,481, \mu=2,5 \times 10^{-9}\right.$, $M=5)$

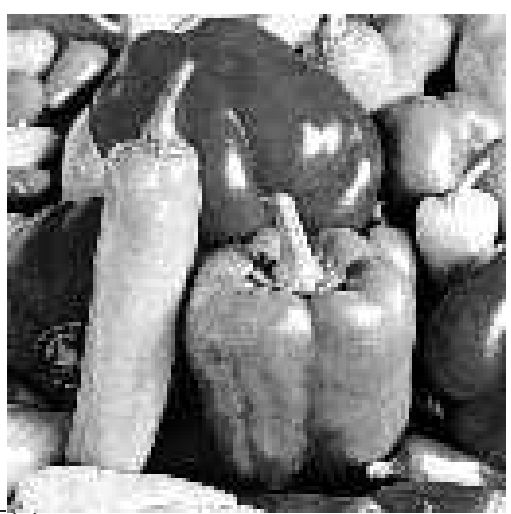

(f) Imagem restaurada com a combinação (\%MSE $=0,451, \mu_{1}=2,5 \times$ $10^{-9}, \mu_{2}=5,0 \times 10^{-2}, \mu_{\alpha}=10^{-3}$, $M_{1}=5, M_{2}=9$ )

Fonte: Autora. 
Figura 52 - Similaridade estrutural média (MSSIM) de cada algoritmo e valor médio do parâmetro de mistura $\bar{\lambda}(c)$ ao longo dos ciclos de varredura para a restauração da imagem Peppers 5-bits/pixel, degradada pela PSF $\mathbf{H}_{4}$ da Tabela 9 (página 83).
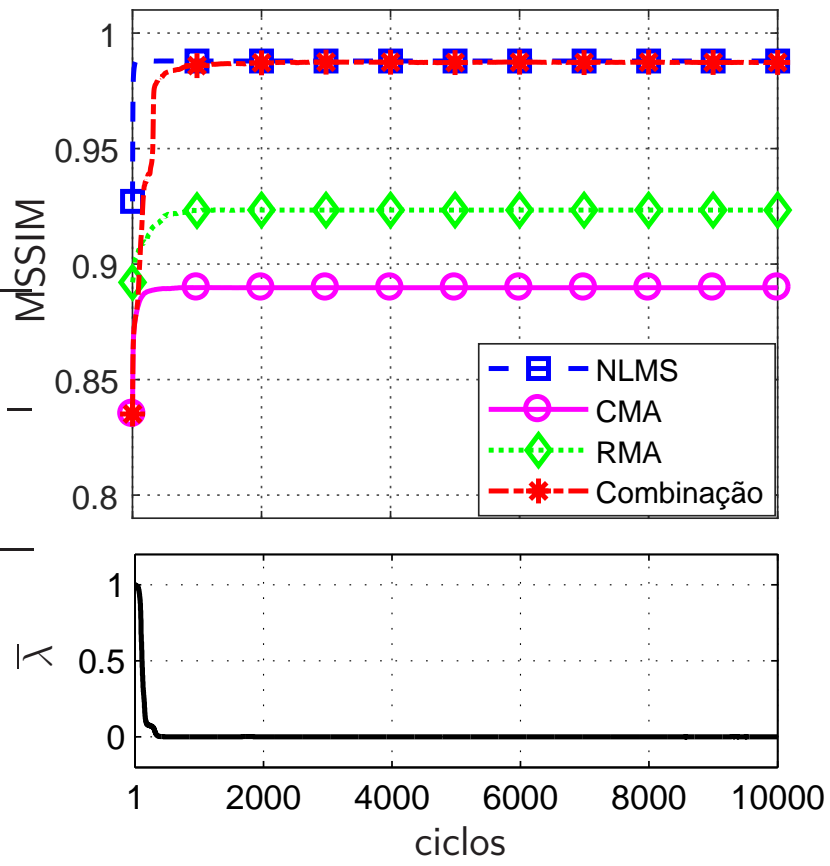

Fonte: Autora.

O MSSIM dos algoritmos é mostrado na Figura 54 em função do ciclo de varredura. O MSSIM da imagem degradada era de 0,7092 e ao final dos $10^{4}$ ciclos, os valores alcançados pelos algoritmos foram: 0,991 (NLMS), 0,853 (RMA), 0,830 (CMA) e 0,984 (combinação). Novamente, graças ao chaveamento automático para o modo de DD, a combinação conseguiu se aproximar do NLMS, como pode ser confirmado pelo parâmetro de mistura médio $\bar{\lambda}(c)$ da Figura 54, que converge para zero após $c=4000$ ciclos, assim como o patamar do MSSIM da combinação converge para um patamar muito próximo ao do NLMS. Qualitativamente, a imagem restaurada pela combinação também apresentou melhores resultados, sendo mais semelhante à imagem original e à restaurada pelo NLMS. Cabe observar também, que a falta de um critério de parada pode causar uma degradação no desempenho do CMA, o que não foi observado nos demais algoritmos. 
Figura 53 - Efeitos da restauração de imagens com filtros adaptativos. Imagem Lenna $128 \times 128$ pixels, 5-bits/pixel, degradada pela PSF $\mathbf{H}_{5}$ da Tabela 9 (página 83).

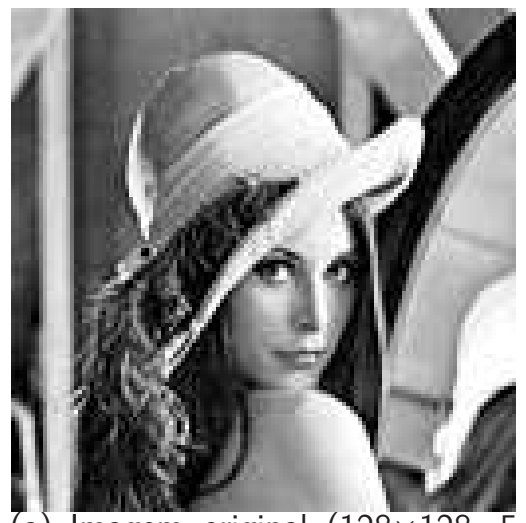

(a) Imagem original $(128 \times 128,5$ bits/pixel)

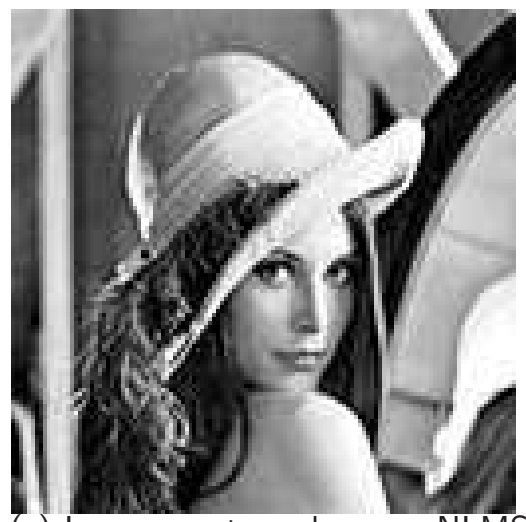

(c) Imagem restaurada com o NLMS

$\left(\% \mathrm{MSE}=0,180, \mu=5,0 \times 10^{-2}\right.$, $M=5)$

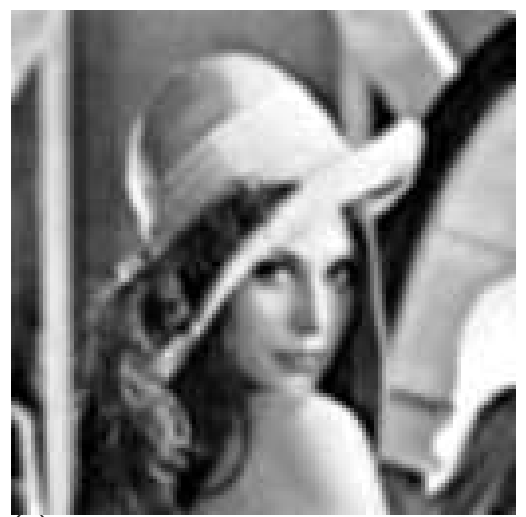

(e) Imagem restaurada com o RMA

$\left(\% \mathrm{MSE}=4,993, \mu=5,0 \times 10^{-4}\right.$, $M=5)$

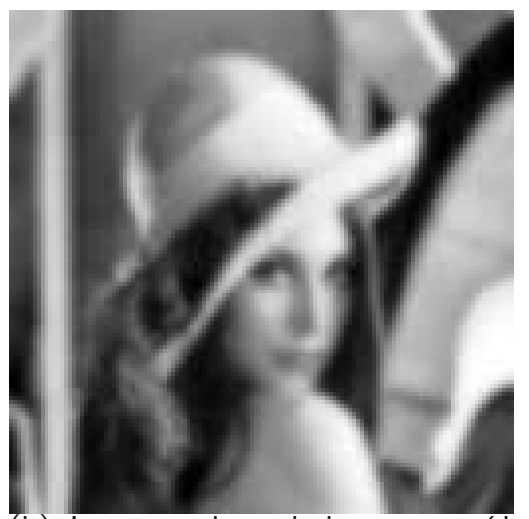

(b) Imagem degradada, sem ruído $(\% \mathrm{MSE}=9,695)$

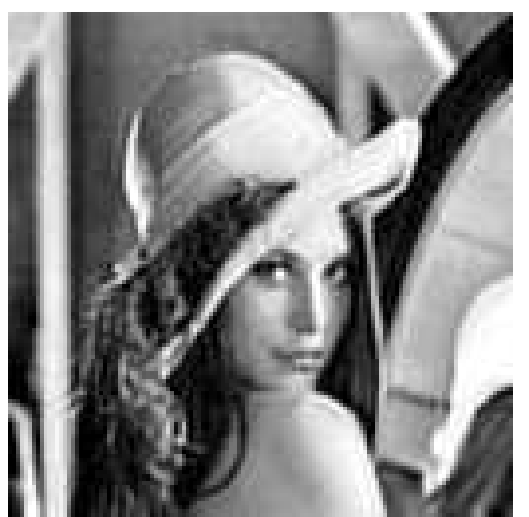

(d) Imagem restaurada com o CMA $\left(\% \mathrm{MSE}=6,520, \mu=10^{-10}, M=5\right)$

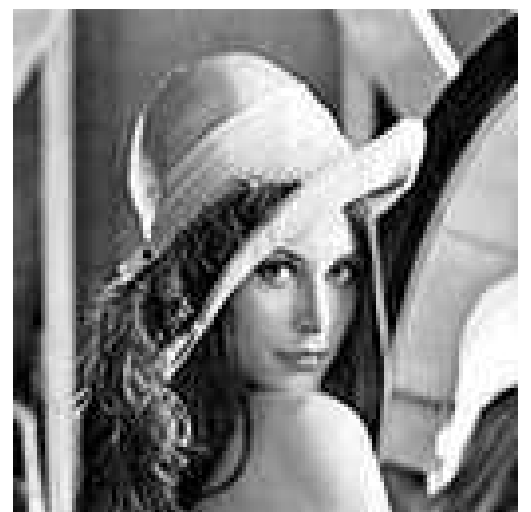

(f) Imagem restaurada com a combinação $\left(\% \mathrm{MSE}=0,277, \mu_{1}=\right.$ $10^{-10}, \mu_{2}=5,0 \times 10^{-2}, \mu_{\alpha}=10^{-4}$, $M_{1}=M_{2}=5$ )

Fonte: Autora. 
Figura 54 - Similaridade estrutural média (MSSIM) de cada algoritmo e valor médio do parâmetro de mistura $\bar{\lambda}(c)$ ao longo dos ciclos de varredura para a restauração da imagem Lenna 5-bits/ pixel, degradada pela PSF $\mathbf{H}_{5}$ da Tabela 9 (página 83).
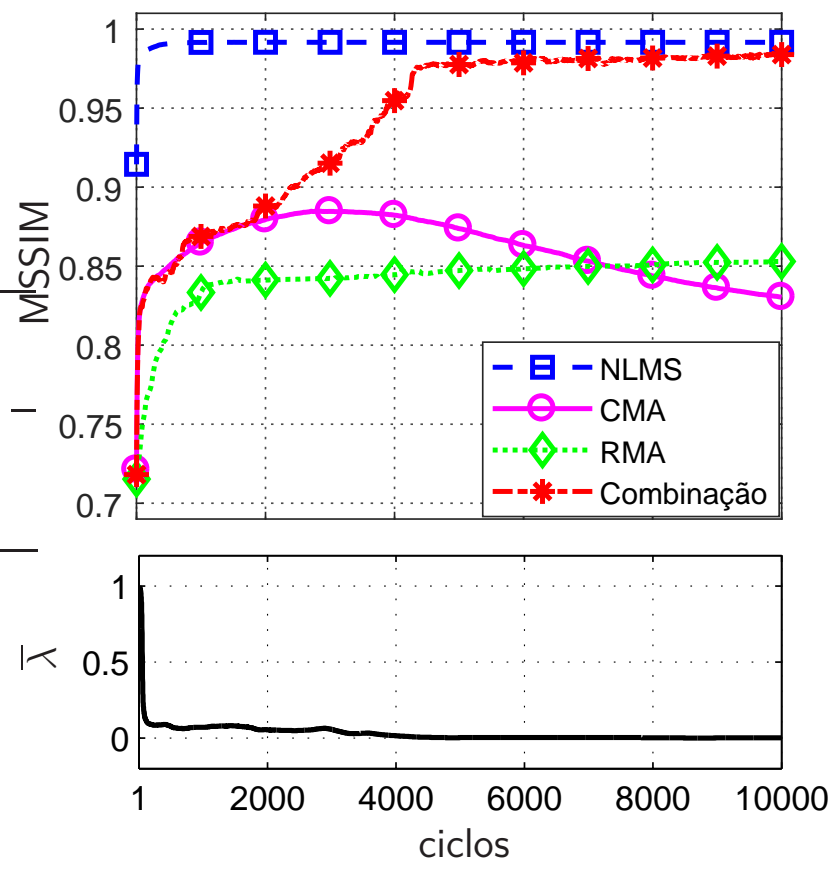

Fonte: Autora.

Os resultados da combinação são comparados também com os do algoritmo de desconvolução cega provido pela função deconvblind do MATLAB, que estima simultaneamente a PSF e a imagem original. Essa função implementa os métodos de (BIGGS; ANDREWS, 1997) e (JANSSON, 1996), baseados na estimação de máxima verossimilhança para obter iterativamente a estimativa da PSF, enquanto utiliza o algoritmo iterativo de Richardson-Lucy (RICHARDSON, 1972; LUCY, 1974) para estimar a imagem original. Uma limitação dessa função, é a dimensão escolhida para a PSF como parâmetro de entrada da função, pois quanto maior for esse valor, pior será a qualidade da estimativas da PSF.

Nas Figuras 55, 56 e 57 são mostrados os resultados da função deconvblind comparados aos da combinação para cada um dos três casos discutidos, onde se utilizou a dimensão $3 \times 3$ para todas as PSFs estimadas, pois esse método obtem melhores resultados quando essa dimensão não é grande. O número de iterações do algoritmo para cada imagem foi escolhido de forma a obter-se a imagem recuperada com melhor valor de \%MSE e MSSIM. Como a 
atualização de ambos algoritmos é feita de forma diferente ao longo das iterações, escolheu-se mostrar apenas o valor final do MSSIM de cada algoritmo na Tabela 10, juntamente com os valores de \%MSE. Em todos esses casos, o desempenho da combinação foi melhor. A mesma conclusão também pode ser obtida comparando qualitativamente as imagens resultantes de cada algoritmo.

Figura 55 - Resultado do algoritmo de desconvolução cega do MATLAB, deconvblind, para a imagem Cameraman $128 \times 128$ pixels, 4-bits/pixel, degradada pela PSF $\mathbf{H}_{3}$ da Tabela 9 (página 83).

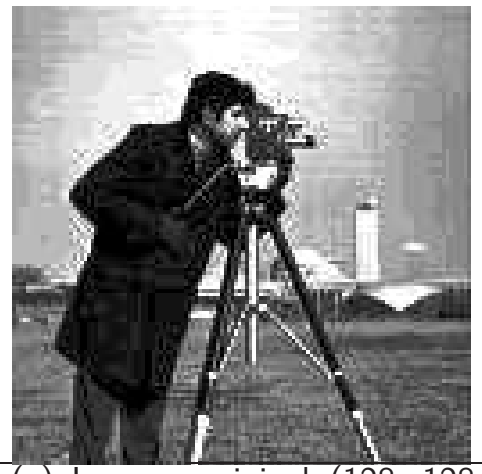

(a) Imagem original $(128 \times 128$, 4-bits/pixel)

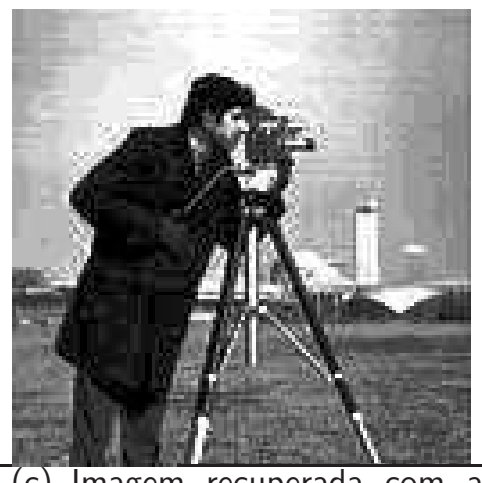

(c) Imagem recuperada com a combinação (\%MSE $=0,402$, MSSIM $=0,990)$

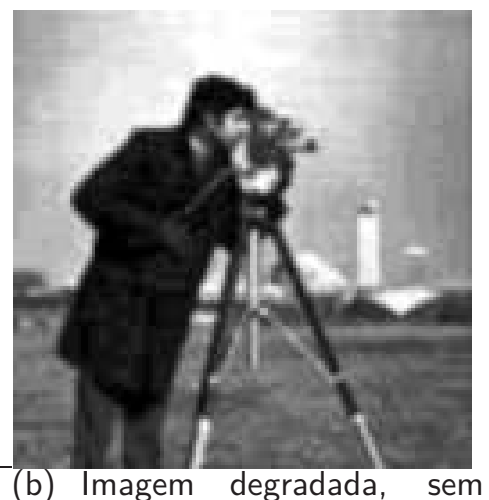

ruído $(\% \mathrm{MSE}=4,874, \mathrm{MSSIM}$ $=0,766)$

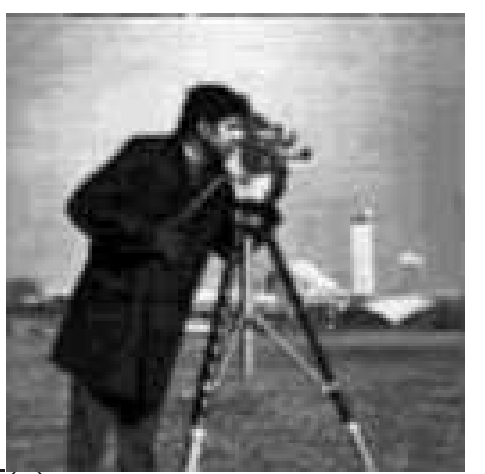

(d) Imagem recuperada com o algoritmo deconvblind (\%MSE = $5,120, \mathrm{MSSIM}=0,519)$

Fonte: Autora.

Os resultados da função deconvblind foram piores nas imagens Cameraman e Lenna, pois o desempenho dessa solução tende a piorar com o aumento da dimensão da PSF, que nesses casos foram $5 \times 5$ e $9 \times 9$, respectivamente. Já na imagem Peppers, como a dimensão da PSF foi $3 \times 3$, a função obteve um resultado melhor, chegando próximo ao da combinação. Cabe 
lembrar que as PSFs utilizadas nestas simulações, $\mathbf{H}_{3}, \mathbf{H}_{4}$ e $\mathbf{H}_{5}$, são consideradas mais "fáceis" de serem mitigadas por um LTE. Assim, para esses tipos de PSFs, a combinação mostrou-se uma solução mais apropriada que o algoritmo da função deconvblind na restauração cega de imagens.

Figura 56 - Resultado do algoritmo de desconvolução cega do MATLAB, deconvblind, para a imagem Peppers $128 \times 128$ pixels, 5-bits/ pixel, degradada pela PSF $\mathbf{H}_{4}$ da Tabela 9 (página 83).

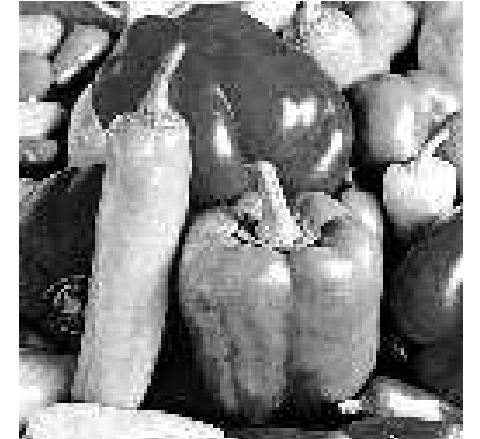

(a) Imagem original $(128 \times 128$, 5-bits/pixel)

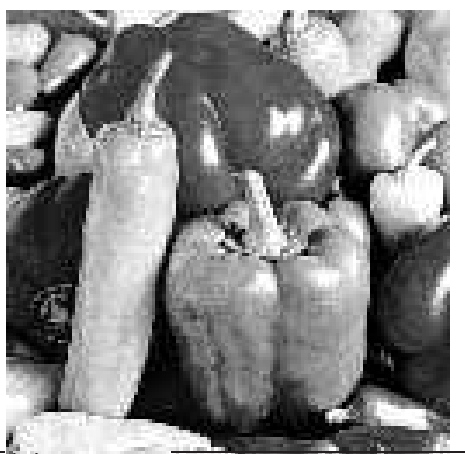

(c) Imagem recuperada com a combinação (\%MSE $=0,451$, $\operatorname{MSSIM}=0,987)$

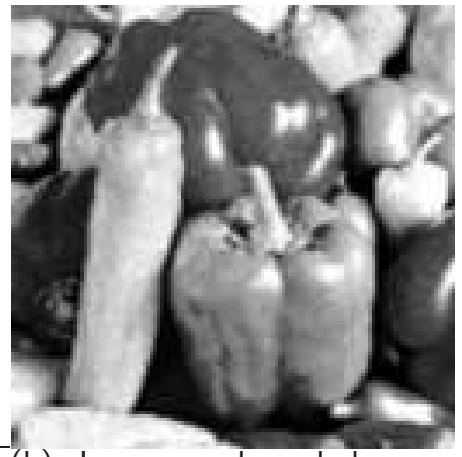

(b) Imagem degradada, sem ruído $(\% \mathrm{MSE}=3,227, \mathrm{MSSIM}$ $=0,906)$

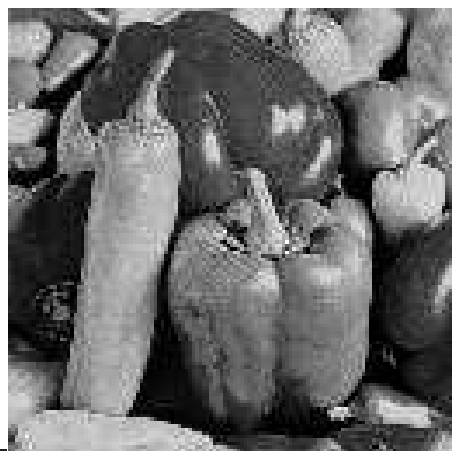

(d) Imagem recuperada com 0 algoritmo deconvblind (\%MSE $=0,475, \mathrm{MSSIM}=0,933)$

Fonte: Autora.

Comparando os resultados da combinação com os da equalização dos canais da Tabela 4 do Capítulo 3 (página 37), "equivalentes" às PSFs usadas nesta seção, verifica-se que é possível obter uma relação entre as PSFs e os canais de comunicação, uma vez que um canal proveniente de uma PSF considerada "fácil" de ser revertida por um filtro, também pode ser considerado fácil de ser equalizado por um filtro. No entanto, cabe lembrar que o problema da 
restauração com filtros é relativamente diferente da equalização, pois o vetor de entrada não é regressor e são necessárias sucessivas varreduras sobre a mesma imagem para a convergência do algoritmo, o que pode dificultar às vezes a comparação entre essas duas abordagens.

Figura 57 - Resultado do algoritmo de desconvolução cega do MATLAB, deconvblind, para a imagem Lenna $128 \times 128$ pixels, 5-bits/pixel, degradada pela PSF $\mathbf{H}_{5}$ da Tabela 9 (página 83).

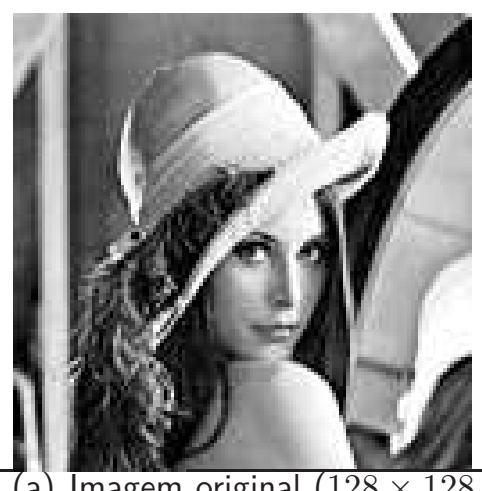

(a) Imagem original $(128 \times 128$, 5-bits/pixel)

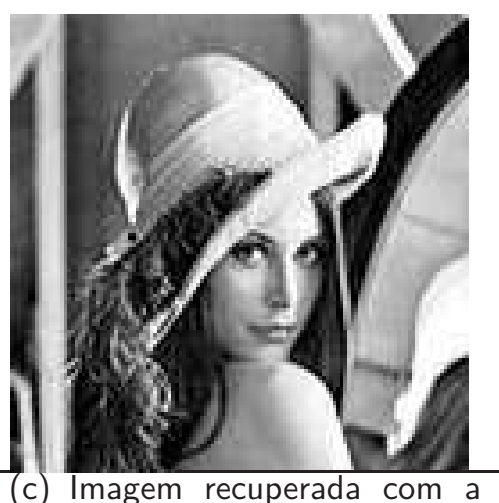

(c) Imagem recuperada com a combinação (\%MSE $=2,777$, $\mathrm{MSSIM}=0,984$ )

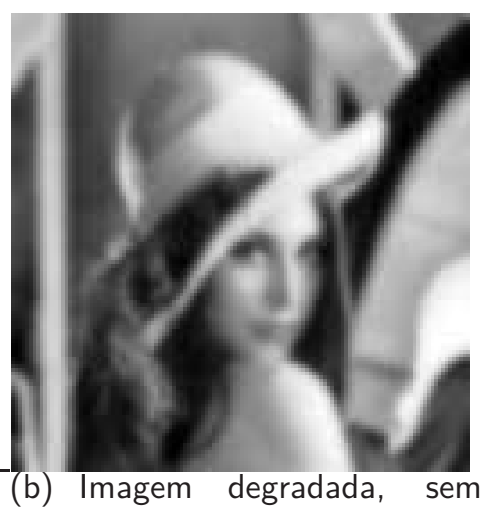

ruído (\%MSE = 9,695, MSSIM $=0,709)$

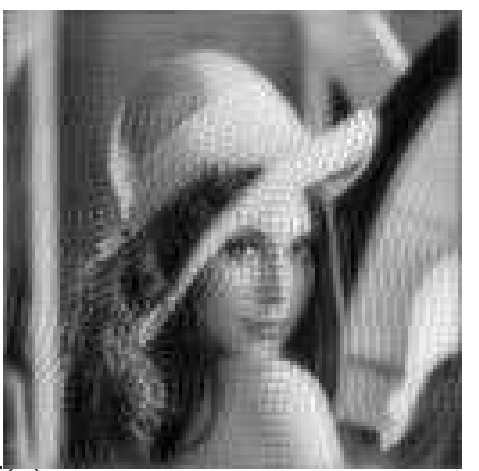

(d) Imagem recuperada com o algoritmo deconvblind (\%MSE = $2,473, \mathrm{MSSIM}=0,711$ )

Fonte: Autora. 
Tabela 10: Valores de MSSIM e \%MSE para a restauração cega com a função do MATLAB deconvblind e com a combinação do CMA com NLMS-DD.

\begin{tabular}{ccccc}
\hline \multirow{2}{*}{ Imagem } & \multicolumn{2}{c}{ deconvblind } & \multicolumn{2}{c}{ Combinação } \\
& \%MSE & MSSIM & \%MSE & MSSIM \\
\hline Cameraman & 5,120 & 0,519 & 0,402 & 0,990 \\
Peppers & 0,475 & 0,933 & 0,451 & 0,987 \\
Lenna & 2,473 & 0,711 & 2,777 & 0,984 \\
\hline
\end{tabular}

Fonte: Autora.

A combinação do CMA com o NLMS-DD que usa o erro do CMA $\varepsilon(n)$ para adaptar a variável auxiliar $\alpha(n)$ foi descartada para os casos apresentados por não proporcionar um bom comportamento na restauração. Como exemplo desse comportamento insatisfatório, são mostradas na Figura 58 as curvas do MSSIM e do parâmetro $\bar{\lambda}(c)$ para o caso da primeira simulação, a restauração da imagem Cameraman degradada pela PSF $\mathbf{H}_{3}$. Os parâmetros da combinação com o $\varepsilon(n)$ foram os mesmos usados na combinação com $e_{d}(n)$, variando apenas o passo $\mu_{\alpha}=10^{-6}$. Embora a curva do MSSIM do NLMS-DD apresente valores superiores aos do CMA, esses valores não chegam a ser muito diferentes como na combinação com $e_{d}(n)$. Além disso, a combinação não chaveia para o NLMS-DD, pois $\bar{\lambda}(c)$ é aparentemente constante (na média) em todos os ciclos. Esse comportamento também foi observado para outros valores de $\mu_{\alpha}$ e indica que a combinação não funcionou como deveria. Portanto, acredita-se que a minimização do erro do CMA não é um critério adequado para a restauração dessas imagens. 
Figura 58 - Similaridade estrutural média (MSSIM) da combinação com o erro $\varepsilon(n)$ e valor médio do parâmetro de mistura $\bar{\lambda}(c)$ ao longo dos ciclos de varredura para restauração da imagem Cameraman 4-bits/pixel degradada pela PSF $\mathbf{H}_{3}$ da Tabela 9 (página 83).
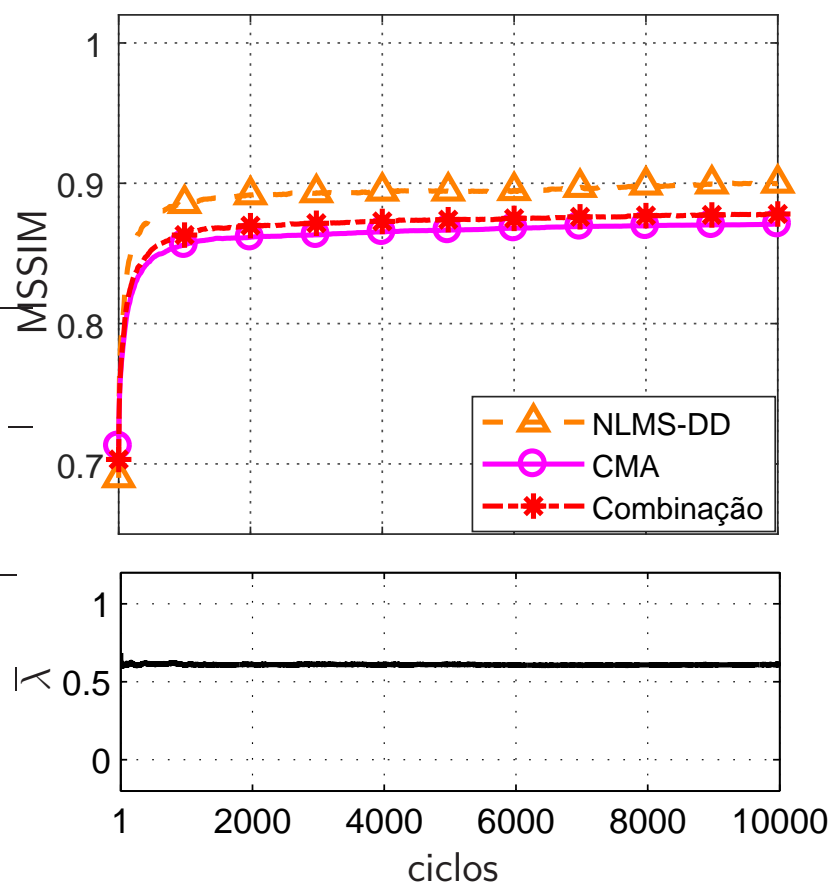

Fonte: Autora.

É importante ressaltar que resultados preliminares indicam que o parâmetro de mistura médio $\bar{\lambda}(c)$ pode ser usado como critério de parada do esquema, de maneira que, a partir desse ponto ocorra o chaveamento para o equalizador DD, evitando uma eventual degradação de desempenho e reduzindo o tempo de execução do algoritmo. A Figura 59 mostra dois valores de critério de parada para a terceira simulação, que usa a imagem Lenna, $128 \times 128$, 5-bits/pixel, degradada pela PSF $\mathbf{H}_{5}$. A Figura $60($ a) mostra que $\bar{\lambda}(c) \leq 0,06$ é um bom critério, uma vez que o MSSIM se mantém quase o mesmo desempenho de quando o critério não é usado. A Figura 60 (b), por sua vez, mostra que $\bar{\lambda}(c) \leq 0,07$ não é uma boa escolha, pois o MSSIM torna-se muito ruim, indicando que para essa condição, o NLMS-DD não consegue convergir sem a ajuda do CMA. 
Figura 59 - MSSIM das saídas da combinação para um valor específico de $\bar{\lambda}$ como critério de parada da combinação e consequente chaveamento para NLMS-DD.

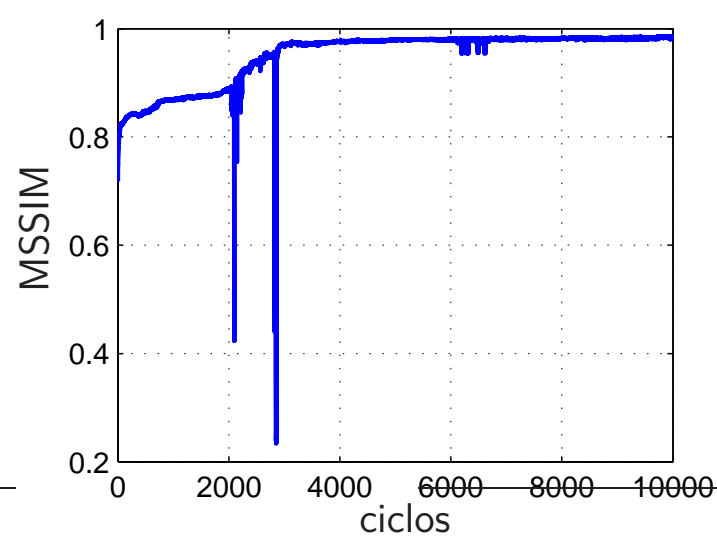

(a) critério de parada: $\bar{\lambda} \leq 0,06$

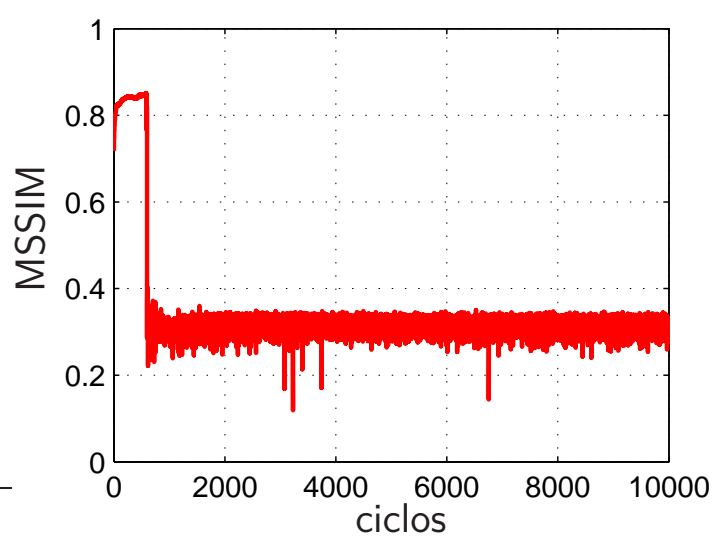

(b) critério de parada: $\bar{\lambda} \leq 0,07$

Fonte: Autora.

\subsubsection{Resultados da combinação do CMA com equalizadores não lineares}

Buscando-se melhorar o desempenho da combinação para PSFs mais complicadas, o esquema da combinação do CMA com o DFE-NLMS-DD foi verificado para a restauração de imagens. Para isso, a combinação deve ser implementada utilizando os procedimentos discutidos na Seção 4.2 e o algoritmo de combinação descrito na Tabela 5 do Capítulo 3 (página 45).

Por meio de simulações, verificou-se que essa combinação é mais suscetível às soluções degeneradas em restauração de imagens que em equalização. Tais soluções foram identificadas observando o vetor de coeficientes $\mathbf{w}_{f b}(n)$, concatenação dos vetores do filtro direto $\mathbf{w}_{f}(n) \mathrm{e}$

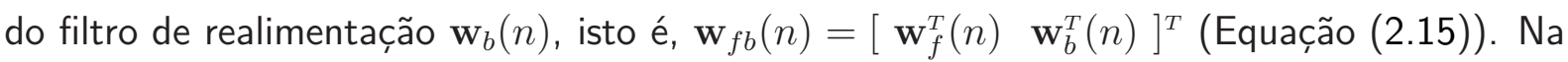
solução degenerada, a saída do DFE deixa de depender da sua entrada, o que significa que o vetor $\mathbf{w}_{b}(n)$ é atualizado sem utilizar a saída do filtro direto. Nesse caso, $\mathbf{w}_{f}(n)=\mathbf{0}$ e o filtro de realimentação recebe as decisões da sua própria saída, resultando em $\mathbf{w}_{b}(n)$ igual a um pulso unitário.

A Figura 60 mostra o vetor $\mathbf{w}_{f b}(n)$ em quatro diferentes momentos durante a restauração da imagem Cameraman, degradada pela PSF $\mathbf{H}_{3}$ da Tabela 9. Nessa simulação, foram utilizados 1000 ciclos de varredura, e os tamanhos dos filtros foram $M_{f}=M_{b}=25$, sendo $M_{f b}=50$. Assim, os gráficos da Figura 60 mostram $\mathbf{w}_{f b}(n)$ para $c=100,250,500$ e 1000 
ciclos, onde $\mathbf{w}_{f}(n)$ corresponde à primeira metade das amostras e $\mathbf{w}_{b}(n)$ à segunda metade. Avaliando a evolução de $\mathbf{w}_{f}(n)$ e $\mathbf{w}_{b}(n)$ durante esses ciclos, percebe-se que as amplitudes dos coeficientes do $\mathbf{w}_{f}(n)$ convergem rapidamente para zero, ao passo que $\mathbf{w}_{b}(n)$ converge para um pulso unitário deslocado, comprovando que esse esquema resultou em uma solução degenerada.

Para este caso, são mostrados na Figura 61, as curvas de MSSIM da combinação e dos seus filtros componentes juntamente com o parâmetro $\bar{\lambda}(c)$ ao longo dos 1000 ciclos de varredura. Nota-se que a combinação chaveia no início para o DFE-NLMS-DD, cujo MSSIM cresce mais rápido que o do CMA. No entanto, a curva do MSSIM do DFE-NLMS-DD torna-se constante após aproximadamente 100 ciclos, assumindo o valor 0,845. Esse comportamento pode ser justificado pelo fato do filtro direto ter deixado de atuar e o filtro de realimentação ter saídas iguais às próprias entradas, resultando sempre no mesmo sinal de imagem.

Assim, acredita-se que as soluções degeneradas são fruto do uso da decisão global da combinação como sinal desejado do DFE e que ocorram com mais frequência em imagens, devido às características inerentes ao problema, como o fato do sinal de imagem apresentar menos diversidade que um sinal de comunicação, ou o vetor de entrada do filtro não ser regressor. Um comportamento similar ao do MSSIM da Figura 61 também foi verificado para a restauração com a combinação do CMA com o QKLMS-DD, o que sugere que esse esquema possa ter apresentado soluções degeneradas, uma vez que estas soluções foram observadas em equalização, como foi mostrado na Seção 3.2.3. Portanto, o uso de critérios de restrição no erro de decisão da combinação com o DFE ou QKLMS pode aprimorar o desempenho dessas soluções e merece ser mais bem investigado em trabalhos futuros. 
Figura 60 - Representação dos vetores de coeficientes $\mathbf{w}_{f b}(n)$ do DFE-NLMS-DD para a restauração da imagem Cameraman, $128 \times 128$, 4-bits/pixel degradada pela PSF $\mathbf{H}_{3}$ da Tabela 9. Para $c=100,250,500$ e 1000 ciclos de varredura.
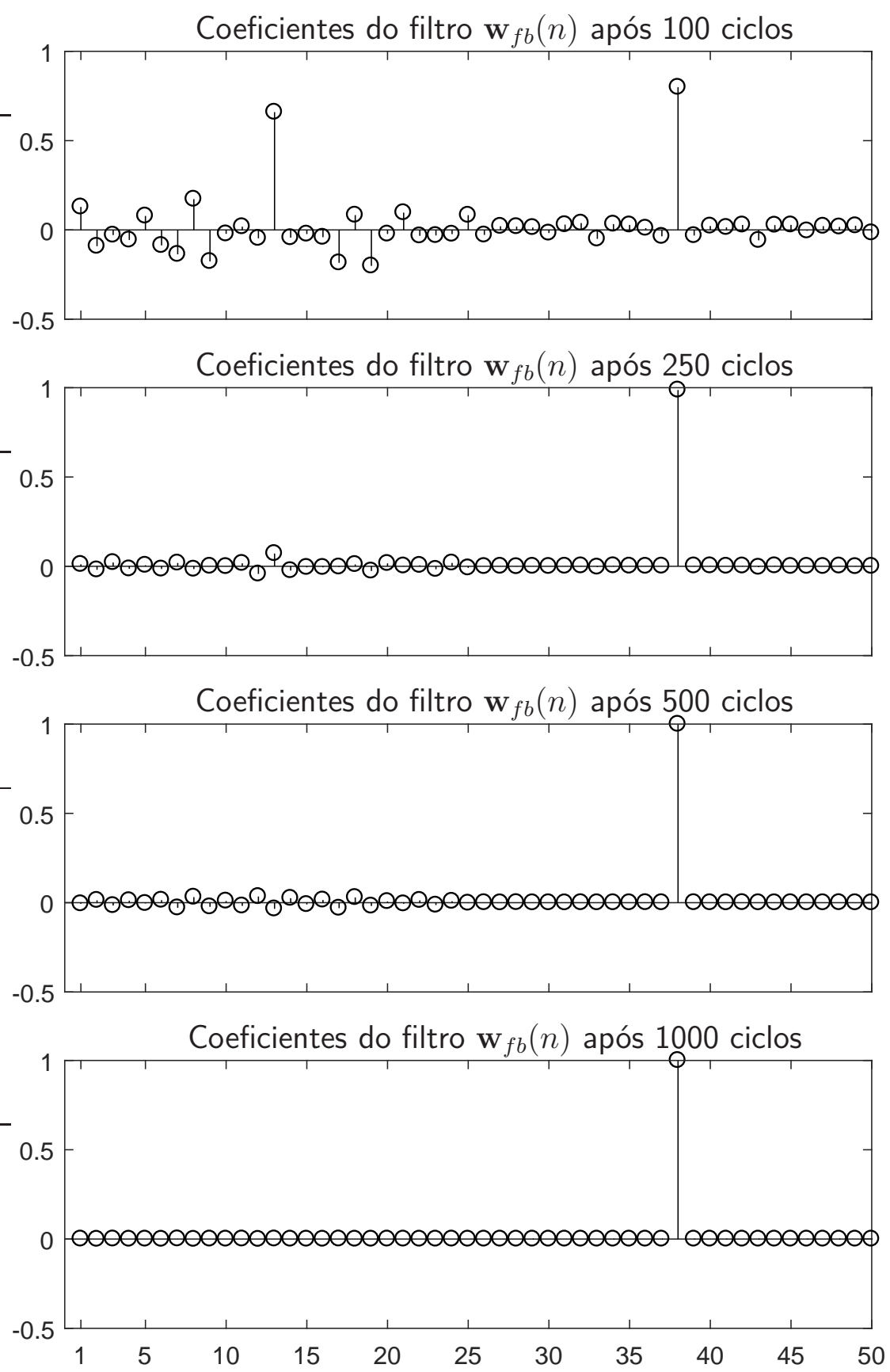

Fonte: Autora. 
Figura 61 - Similaridade estrutural média (MSSIM) e valor médio do parâmetro de mistura $\bar{\lambda}(c)$ para a combinação do CMA com o DFE-NLMS-DD para a restauração da imagem Cameraman 4-bits/ pixel degradada pela PSF $\mathbf{H}_{3}$ da Tabela 9.

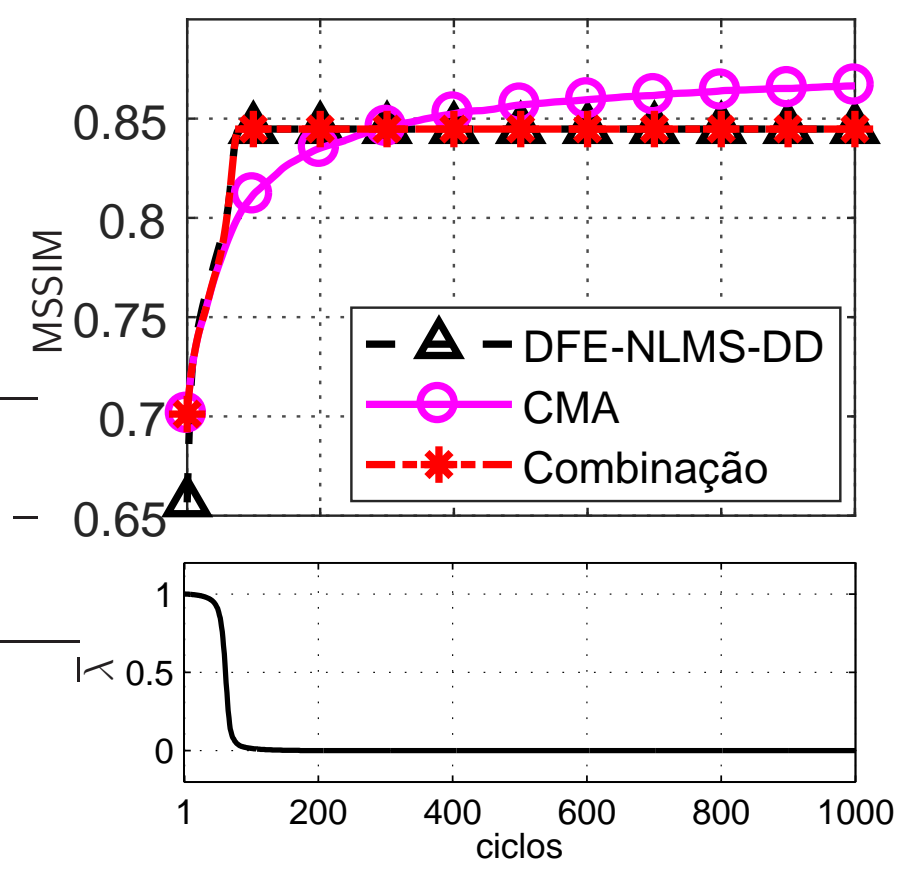

Fonte: Autora.

\subsection{Conclusão}

Neste capítulo, o esquema da combinação convexa entre um equalizador cego e um equalizador no modo DD é estendido para a restauração cega de imagens. Os comportamentos da combinação do CMA com o NLMS-DD e da combinação do CMA com o DFE-NLMS-DD foram verificados por meio de simulações numéricas. Os resultados das simulações mostram que a combinação com o NLMS-DD pode apresentar desempenho superior ao dos algoritmos cegos CMA e RMA e próximo ao do NLMS supervisionado. Além disso, para os modelos de degradação utilizados, esse esquema demonstrou relativa vantagem em relação ao algoritmo de desconvolução cega baseado na estimação de máxima verossimilhança e no algoritmo de Richardson-Lucy. A combinação com o NLMS-DD mostrou-se mais eficiente em casos em que a degradação da imagem é representada por uma PSF simétrica com maior concentração de energia nas suas posições centrais, podendo ser relacionada a um canal de comunicação cuja a resposta ao pulso unitário é simétrica e o elemento central possui maior amplitude. Nesses 
casos, soluções com o LTE são suficientes para equalizar o canal, o que aparentou ser válido também para a restauração de imagens. Já a combinação com o DFE-NLMS-DD, alternativa ao LTE em PSFs mais difíceis, apresentou soluções degeneradas até mesmo para degradações consideradas simples, o que indica que a minimização do erro de decisão ao quadrado talvez não seja adequado para este problema, sugerindo a investigação de novas alternativas. 


\section{CONCLUSÕES}

A restauração cega de imagens abrange diferentes tipos de abordagens. Muitas delas utilizam modelos probabilísticos para a imagem original, função de degradação e imagem observada, o que permite o uso de diferentes estratégias, como a inferência por máxima verossimilhança e a máxima probabilidade a posteriori, para estimar a imagem original e a degradação. Neste trabalho, a abordagem utilizada é proveniente da equalização adaptativa de canais de comunicação, onde são usados filtros adaptativos para a recuperação de um sinal de comunicação degradado por um canal. Dessa forma, ao tratar a imagem como um sinal de comunicação, por meio do mapeamento da mesma em um sinal $N$-PAM, é possível utilizar técnicas de equalização cega para estimar a imagem original.

$\mathrm{Na}$ investigação de soluções para restauração, partiu-se do estudo de algoritmos adaptativos para equalização, que são descritos no Capítulo 2. Esses algoritmos são divididos entre soluções lineares e não lineares. As soluções lineares são suficientes nos casos em que a degradação é simples, e as não lineares abrangem degradações mais complicadas.

No Capítulo 3, discute-se o esquema baseado na combinação convexa entre um equalizador no modo cego e um equalizador no modo de decisão direta. Esse esquema garante o chaveamento automático entre o modo cego e o DD, reduzindo o MSE na saída do equalizador. No mesmo capítulo, propõe-se o uso das soluções não lineares discutidas anteriormente para o equalizador DD, estendendo a aplicação da combinação para canais mais complicados, como os com nulos espectrais e os não lineares. As simulações mostraram que no geral ambos esquemas podem apresentar um bom desempenho em equalização, o que leva a crer que tais soluções podem ser utilizadas na restauração de imagens. No entanto, em alguns casos o uso de soluções não lineares resultou em soluções degeneradas, que são fruto do uso do critério cego minimizado pela combinação.

Assim, no Capítulo 4, o esquema de combinação foi estendido para o uso em imagens. As simulações para a combinação com soluções lineares apresentaram resultados com desempenho de um algoritmo de filtragem adaptativa supervisionada, sendo superior a outras soluções adaptativas cegas. Além disso, esse esquema se mostrou mais eficiente em degradações cuja a PSF possui características semelhantes às de um canal de comunicação considerado simples. Já 
a combinação com as soluções não lineares mostrou-se mais suscetível às soluções degeneradas que em equalização, o que pode ser fruto desses problemas serem de naturezas distintas, pois o fato do sinal de imagem apresentar menor diversidade que o sinal de comunicação e do vetor de entrada não ser regressor dificulta a filtragem adaptativa em imagens, podendo resultar em comportamentos diferentes dos verificados em equalização. Assim, o uso de restrições no critério de minimização da combinação merece ser melhor investigado para evitar essas soluções.

Ainda, a minimização do erro de decisão pela combinação não é o critério mais adequado para a restauração de imagens, uma vez que o mesmo não reflete as características da imagem relacionada à percepção humana, o que significa que a combinação pode funcionar em termos de MSE, mas isso não garante que a qualidade da imagem recuperada seja realmente boa. Portanto, é importante em trabalhos futuros, buscar-se novas alternativas de adaptação para a combinação.

Dessa maneira, algumas sugestões para trabalhos futuros são:

- Compreender com maior profundidade o surgimento de soluções degeneradas na combinação com o equalizador de decisão realimentada para imagens, e com isso buscar novas alternativas de restrição para a função custo minimizada pela combinação;

- Compreender melhor o comportamento da combinação com o filtro adaptativo baseado em kernel para imagens, verificando a existência de soluções degeneradas e se for o caso, propor novas alternativas de restrição para a função custo;

- Por fim, de uma maneira geral, investigar outros tipos de função custo para a combinação, uma vez que o erro de decisão não é a medida mais adequada para representar a qualidade de uma imagem. Além disso, espera-se que o uso de critérios que levem em conta as características estatísticas do sinal de imagem possa melhorar o desempenho da restauração. 


\section{REFERÊNCIAS}

ABREU, R.; SILVA, M. T. M. A multimodulus algorithm for blind image deconvolution. In: Proc. of International Workshop on Telecommunications (IWT). Rio de Janeiro, Brazil: [s.n.], 2011. p. 35-41.

ABREU, R. A. Técnicas de equalização de canais de comunicação aplicadas a imagens. Dissertação (Dissertação de Mestrado em Engenharia Elétrica) - Escola Politécnica da Universidade de São Paulo, São Paulo, 2011.

ADAM, D.; MICHAILOVICH, O. Blind deconvolution of ultrasound sequences using nonparametric local polynomial estimates of the pulse. IEEE Transactions on Biomedical Engineering, v. 49, n. 2, p. 118-131, Feb 2002. ISSN 0018-9294.

ALMEIDA, M. S. C.; ALMEIDA, L. B. Blind deblurring of natural images. In: 2008 IEEE International Conference on Acoustics, Speech and Signal Processing. [S.I.: s.n.], 2008. p. 1261-1264. ISSN 1520-6149.

Blind and semi-blind deblurring of natural images. IEEE Transactions on Image Processing, v. 19, n. 1, p. 36-52, Jan 2010. ISSN 1057-7149.

ALMEIDA, M. S. C.; FIGUEIREDO, M. A. T. Parameter estimation for blind and non-blind deblurring using residual whiteness measures. IEEE Transactions on Image Processing, v. 22, n. 7, p. 2751-2763, 2013. ISSN 1057-7149.

AMIZIC, B. et al. Sparse bayesian blind image deconvolution with parameter estimation. In: 2010 18th European Signal Processing Conference. [S.I.: s.n.], 2010. p. 626-630. ISSN 2219-5491.

ANDRÉ, N. S. et al. Adaptive nonlinear volterra equalizer for mitigation of chirp-induced distortions in cost effective imdd ofdm systems. Opt. Express, OSA, v. 21, n. 22, p. 26527-26532, Nov 2013. Disponível em: <http://www.opticsexpress.org/abstract.cfm? $\mathrm{URI}=\mathrm{oe}-21-22-26527>$.

ARENAS-GARCÍA, J.; FIGUEIRAS-VIDAL, A. R.; SAYED, A. H. Mean-square performance of a convex combination of two adaptive filters. IEEE Transactions on Signal Processing, v. 54, p. 1078-1090, Mar. 2006.

ARENAS-GARCÍA, J.; GÓMEZ-VERDEJO, V.; FIGUEIRAS-VIDAL, A. R. New algorithms for improved adaptive convex combination of LMS transversal filters. IEEE Transactions on Instrumentation and Measurement, v. 54, p. 2239-2249, Dec. 2005.

AUSTIN, M. Decision Feedback Equalization for digital communication over dispersive channels. MIT Research Laboratory of Electronics Technical Report, n. 461, Aug. 1967. 
AZPICUETA-RUIZ, L. A.; FIGUEIRAS-VIDAL, A. R.; ARENAS-GARCÍA, J. A normalized adaptation scheme for the convex combination of two adaptive filters. In: Proc. IEEE Int. Conf. Acoustics, Speech, and Signal Process. Las Vegas, NV: [s.n.], 2008. p. 3301-3304.

BANHAM, M. R.; KATSAGGELOS, A. K. Digital image restoration. IEEE Signal Processing Magazine, v. 14, n. 2, p. 24-41, 1997. ISSN 1053-5888.

BERTERO, M.; BOCCACCI, P. Introduction to Inverse Problems in Imaging. [S.I.]: Institute of Physics Publishing, 1998. Image restoration methods for the large binocular telescope (lbt). v. 147, 122000.

BIGGS, D. S. C.; ANDREWS, M. Acceleration of iterative image restoration algorithms. Appl. Opt., OSA, v. 36, n. 8, p. 1766-1775, Mar 1997. Disponível em: <http://ao.osa.org/ abstract.cfm?URI=ao-36-8-1766>.

BORYS, A. Nonlinear Aspects of Telecommunications: Discrete Volterra Series and Nonlinear Echo Cancellation. [S.I.]: CRC Press, Inc., 2001.

BRASIL-SILVA, D.; SILVA, M. Um esquema para restauração cega de imagens baseado em combinação de algoritmos adaptativos. Simpósio Brasileiro de Telecomunicações e Processamento de Sinais, 2017.

BRONSTEIN, A. M. et al. Quasi-maximum likelihood blind deconvolution of images acquired through scattering media. In: 2004 2nd IEEE International Symposium on Biomedical Imaging: Nano to Macro (IEEE Cat No. 04EX821). [S.I.: s.n.], 2004. p. 352-355 Vol. 1.

BRONSTEIN, M. M. et al. Blind deconvolution of images using optimal sparse representations. IEEE Transactions on Image Processing, v. 14, n. 6, p. 726-736, June 2005. ISSN 1057-7149.

BURSE, K.; YADAV, R. N.; SHRIVASTAVA, S. C. Channel equalization using neural networks: A review. IEEE Transactions on Systems, Man, and Cybernetics, Part C (Applications and Reviews), v. 40, n. 3, p. 352-357, May 2010. ISSN 1094-6977.

CAMPISI, P.; EGIAZARIAN, K. Blind image deconvolution. Theory and applications. [S.I.]: CRC Press, Taylor \& Francis Group, 2007. Contribution: organisation=sgn,FACT1=1. ISBN 0-8493-7367-0.

CASTRO, F. C. C. D.; CASTRO, M. C. F. D.; ARANTES, D. S. Concurrent blind deconvolution for channel equalization. In: Proc. of ICC'2001. [S.I.: s.n.], 2001. v. 2, p. 366-371.

CHAN, T. F.; WONG, C.-K. Total variation blind deconvolution. IEEE Transactions on Image Processing, v. 7, n. 3, p. 370-375, Mar 1998. ISSN 1057-7149.

CHEN, B. et al. Quantized kernel least mean square algorithm. IEEE Transactions on Neural Networks and Learning Systems, v. 23, n. 1, p. 22-32, Jan 2012. ISSN 2162-237X. 
CHEN, S. Low complexity concurrent constant modulus algorithm and soft directed scheme for blind equalization. IEE Proceedings - Vision, Image, and Signal Processing, v. 150, p. 312-320, Oct. 2003.

CHEN, S. et al. Adaptive equalization of finite non-linear channels using multilayer perceptrons. Signal Processing, v. 20, p. 107-119, 1990.

CRIPPS, S. C. RF Power Amplifiers for Wireless Communications, Second Edition (Artech House Microwave Library (Hardcover)). Norwood, MA, USA: Artech House, Inc., 2006. ISBN 1596930187.

DAS, G.; PATTNAIK, P. K.; PADHY, S. K. Artificial neural network trained by particle swarm optimization for non-linear channel equalization. Expert Systems with Applications, v. 41, n. 7, p. 3491 - 3496, 2014. ISSN 0957-4174. Disponível em: < http://www.sciencedirect. com/science/article/pii/S0957417413008701>.

DING, Z.; JOHNSON, C. R.; KENNEDY, R. A. On the (non)existence of undesirable equilibria of godard blind equalizers. IEEE Transactions on Signal Processing, v. 40, n. 10, p. 2425-2432, Oct 1992. ISSN 1053-587X.

DING, Z.; LI, Y. Blind Equalization and Identification. [S.I.]: Marcel Dekke, 2001.

FERGUS, R. et al. Removing camera shake from a single photograph. ACM Trans. Graph., v. 25, p. $787-794,2006$.

FIGUEIRAS-VIDAL, A. Digital Signal Processing in Telecommunications:

European Project COST\#229 Technical Contributions. Springer London,

2012. ISBN 9781447110194. Disponível em: <https://books.google.com.br/books? id $=$ Z4zhBwAAQBAJ $>$.

FORNEY, G. Maximum-likelihood sequence estimation of digital sequences in the presence of intersymbol interference. IEEE Transactions on Information Theory, v. 18, n. 3, p. 363-378, May 1972. ISSN 0018-9448.

FU, X. et al. Superresolution imaging using constrained iterative deconvolution. In: 2016 IEEE 11th Conference on Industrial Electronics and Applications (ICIEA). [S.I.: s.n.], 2016. p. 669-672.

GHOSH, M. Blind decision feedback equalization for terrestrial television receivers.

Proceedings of IEEE, v. 86, p. 2070-2081, Oct. 1998.

GIBSON, G. J.; COWAN, C. F. N.; GRANT, P. M. On the decision regions of multilayer perceptrons. Proceedings of IEEE, v. 78, p. 1590-1599, Oct. 1990.

GIBSON, G. J.; SIU, S.; COWAN, C. F. N. The application of nonlinear structures to the reconstruction of binary signals. v. 39, n. 8, p. 1877-1884, Aug. 1991. ISSN 1053-587X.

GODARD, D. N. Self-recovering equalization and carrier tracking in two dimensional data communication system. v. 28, p. 1867-1875, Nov. 1980.

GOLDSTEIN, A.; FATTAL, R. Blur-kernel estimation from spectral irregularities. In: Proceedings of the 12th European Conference on Computer Vision - Volume Part V. Berlin, Heidelberg: Springer-Verlag, 2012. (ECCV'12), p. 622-635. ISBN 978-3-642-33714-7. Disponível em: <http://dx.doi.org/10.1007/978-3-642-33715-4_45>. 
GONZALEZ, R. C.; WOODS, R. E. Digital Image Processing. 3rd. ed. [S.I.]: Prentice Hall, NJ, 2008.

GUNTURK, B. K.; LI, X. Image Restoration: Fundamentals and Advances. 1st. ed. Boca Raton, FL, USA: CRC Press, Inc., 2017. ISBN 1138071773, 9781138071773.

HADHOUD, M. M.; THOMAS, D. W. The two-dimensional adaptive LMS (TDLMS) algorithm. IEEE Transactions on Circuits and Systems, v. 35, p. 485-494, May 1998.

HAYKIN, S. Adaptive Filter Theory. 4th. ed. [S.I.]: Prentice Hall, Upper Saddle River, 2002.

HOOLE, D. E. Channel equalization for the IS-54 Digital Cellular System with the TMS320C5x. [S.I.]: Texas Instruments, Dallas, 1994.

HUANGPENG, Q. et al. Improved multi-frame super resolution via multiframe blind deblurring using the alternating direction method of multipliers. In: 2015 IEEE International Conference on Progress in Informatics and Computing (PIC). [S.I.: s.n.], 2015. p. 281-285.

JANSSON, P. A. (Ed.). Deconvolution of Images and Spectra (2Nd Ed.). Orlando, FL, USA: Academic Press, Inc., 1996. ISBN 0-12-380222-9.

JEFFERIES, S. M. et al. Blind deconvolution in optical diffusion tomography. Opt. Express, OSA, v. 10, n. 1, p. 46-53, Jan 2002. Disponível em: <http://www.opticsexpress.org/ abstract.cfm?URI=oe-10-1-46>.

JENSEN, J. R.; LULLA, D. K. Introductory digital image processing: A remote sensing perspective. Geocarto International, Taylor \& Francis, v. 2, n. 1, p. 65-65, 1987. Disponível em: <https://doi.org/10.1080/10106048709354084>.

JOHANNISSON, P.; KARLSSON, M. Perturbation analysis of nonlinear propagation in a strongly dispersive optical communication system. Journal of Lightwave Technology, v. 31 , n. 8, p. 1273-1282, April 2013. ISSN 0733-8724.

JOHNSON-JR., C. R. et al. Blind equalization using the constant modulus criterion: a review. Proceedings of the IEEE, v. 86, p. 1927-1950, Oct. 1998.

The core of FSE-CMA behavior theory. In: HAYKIN, S. (Ed.). Unsupervised adaptive filtering: blind equalization. NY: Wiley, 2000. II.

KECHRIOTIS, G.; ZERVAS, E.; MANOLAKOS, E. S. Using recurrent neural networks for adaptive communication channel equalization. IEEE Transactions on Neural Networks, v. 5, p. 267-278, Mar. 1994.

KOFIDIS, E.; RONTOGIANNIS, A. A. Adaptive blast decision-feedback equalizer for mimo-fbmc/oqam systems. In: 21st Annual IEEE International Symposium on Personal, Indoor and Mobile Radio Communications. [S.I.: s.n.], 2010. p. 841-846. ISSN 2166-9570.

KUNDUR, D.; HATZINAKOS, D. Blind image deconvolution. IEEE Signal Processing Magazine, v. 13, p. 43-64, May 1996. 
LÁZARO-GREDILLA, M. et al. Adaptively biasing the weights of adaptive filters. IEEE Transactions on Signal Processing, v. 58, n. 7, p. 3890-3895, Jul. 2010.

LIKAS, A. C.; GALATSANOS, N. P. A variational approach for bayesian blind image deconvolution. IEEE Transactions on Signal Processing, v. 52, n. 8, p. 2222-2233, Aug 2004. ISSN 1053-587X.

LIU, W.; PRÍNCIPE, J. C.; HAYKIN, S. Kernel Adaptive Filtering: A Comprehensive Introduction. [S.I.]: Wiley, 2010.

LUCY, L. B. An iterative technique for the rectification of observed distributions.

Astronomical Journal, v. 79, p. 745-754, June 1974.

MATHEWS, V. J.; SICURANZA, G. L. Polynomial Signal Processing. [S.I.: s.n.], 2000.

MAZO, J. E. Analysis of decision-directed equalizer convergence. Bell Syst. Tech. Journal, v. 59 , n. 10 , p. $1857-1877,1980$.

MENDES-FILHO, J.; MIRANDA, M. D.; SILVA, M. T. M. A regional multimodulus algorithm for blind equalization of QAM signals: introduction and steady-state analysis. Signal Processing, v. 92, n. 11, p. 2643-2656, Nov. 2012.

A regional multimodulus algorithm for blind image deconvolution. In: Proc. IEEE Int. Conf. Image Process. Paris, France: [s.n.], 2014.

MENDES-FILHO, J. et al. A region-based algorithm for blind equalization of QAM signals. In: Proc. of the IEEE/SP 15th Workshop on Statistical Signal Processing. Cardiff, UK: [s.n.], 2009. p. 685-688.

MOLINA, R.; MATEOS, J.; KATSAGGELOS, A. K. Blind deconvolution using a variational approach to parameter, image, and blur estimation. IEEE Transactions on Image Processing, v. 15, n. 12, p. 3715-3727, Dec 2006. ISSN 1057-7149.

MONEY, J. H.; KANG, S. H. Total variation minimizing blind deconvolution with shock filter reference. Image Vision Comput., Butterworth-Heinemann, Newton, MA, USA, v. 26, n. 2, p. 302-314, fev. 2008. ISSN 0262-8856. Disponível em: <http://dx.doi.org/10.1016/j. imavis.2007.06.005>.

OGUNFUNMI, T.; DRULLINGER, T. Equalization of non-linear channels using a volterrabased non-linear adaptive filter. In: 2011 IEEE 54th International Midwest Symposium on Circuits and Systems (MWSCAS). [S.I.: s.n.], 2011. p. 1-4. ISSN 1548-3746.

OLIVEIRA, J. P.; FIGUEIREDO, M. A. T.; BIOUCAS-DIAS, J. M. Parametric blur estimation for blind restoration of natural images: Linear motion and out-of-focus. IEEE Transactions on Image Processing, v. 23, n. 1, p. 466-477, Jan 2014. ISSN 1057-7149.

PAPADIAS, C. B.; PAULRAJ, A. Decision-feedback equalization and identification of linear channels using blind algorithms of the bussgang type. In: Proc. of 29th Asilomar Conf. on Signals, Systems \& Computers. [S.I.: s.n.], 1995. v. 1, p. 335-340.

PARREIRA, W. D. et al. Stochastic behavior analysis of the gaussian kernel least-mean-square algorithm. IEEE Transactions on Signal Processing, v. 60, n. 5, p. 2208-2222, May 2012. ISSN 1053-587X. 
PLATT, J. A resource-allocating network for function interpolation. Neural Computation, v. 3, n. 2, p. 213-225, June 1991. ISSN 0899-7667.

POGGIOLINI, P. et al. The gn-model of fiber non-linear propagation and its applications. Journal of Lightwave Technology, v. 32, n. 4, p. 694-721, Feb 2014. ISSN 0733-8724.

PROAKIS, J.; SALEHI, M. Digital Communications. 5th. ed. [S.I.]: McGraw-Hill, NY, 2007.

QIN, L.; ZHANG, W.-j. A robust decision feedback equalizer for atsc dtv receivers. Journal of Shanghai Jiaotong University (Science), v. 13, n. 1, p. 1-5, Feb 2008. ISSN 1995-8188. Disponível em: <https://doi.org/10.1007/s12204-008-0001-3>.

QURESHI, S. H. Adaptive equalization. Proceedings of the IEEE, v. 73, p. 1349-1387, Sept. 1985.

QURESHI, S. U. H. Adaptive equalization. v. 73, n. 9, p. 1349-1387, Sep. 1985.

RICHARD, C.; BERMUDEZ, J. C. M.; HONEINE, P. Online prediction of time series data with kernels. IEEE Transactions on Signal Processing, v. 57, n. 3, p. 1058-1067, March 2009. ISSN 1053-587X.

RICHARDSON, W. H. Bayesian-based iterative method of image restoration*. Journal of the Optical Society of America, OSA, v. 62, n. 1, p. 55-59, Jan 1972. Disponível em: $<$ http://www.osapublishing.org/abstract.cfm?URI=josa-62-1-55>.

SAYED, A. H. Fundamentals of Adaptive Filtering. [S.I.]: John Wiley \& Sons, NJ, 2003. Adaptive Filters. [S.I.]: John Wiley \& Sons, NJ, 2008.

SCHÖLKOPF, B.; SMOLA, A. Learning with Kernels: Support Vector Machines, Regularization, Optimization, and Beyond. Cambridge, MA, USA: MIT Press, 2002. 644 p. (Adaptive Computation and Machine Learning). Parts of this book, including an introduction to kernel methods, can be downloaded <a href="http://www.learning-withkernels.org/sections/»here $</ a>$.

SHAPIRO, L. G.; STOCKMAN, G. C. Computer Vision. [S.I.]: Prentice Hall, 2001.

SILVA, M. T. M. Equalização não-linear de canais de comunicação. Dissertação (Dissertação de Mestrado em Engenharia Elétrica) - Escola Politécnica da Universidade de São Paulo, São Paulo, 2001.

SILVA, M. T. M.; ARENAS-GARCÍA, J. A soft-switching blind equalization scheme via convex combination of adaptive filters. IEEE Transactions on Signal Processing, v. 61, n. 5, p. 1171-1182, Mar. 2013.

SILVA, M. T. M.; NASCIMENTO, V. H. Tracking analysis of the Constant Modulus Algorithmn. In: Proc. IEEE Int. Conf. Acoustics, Speech, and Signal Process. Las Vegas, NV: [s.n.], 2008. p. 3561-3564.

SZCZECINSKI, L. L.; GEI, A. Blind decision feedback equalisers, how to avoid degenerated solutions. Signal Processing, v. 82, p. 1675-1693, Nov. 2002. 
TOBAR, F. A.; KUNG, S. Y.; MANDIC, D. P. Multikernel least mean square algorithm. IEEE Transactions on Neural Networks and Learning Systems, v. 25, n. 2, p. 265-277, Feb 2014. ISSN 2162-237X.

VAERENBERGH, S. V.; AZPICUETA-RUIZ, L. A.; COMMINIELLO, D. A split kernel adaptive filtering architecture for nonlinear acoustic echo cancellation. In: 2016 24th European Signal Processing Conference (EUSIPCO). [S.I.: s.n.], 2016. p. 1768-1772.

VURAL, C.; SETHARES, W. A. Blind deconvolution of noisy blurred images via dispersion minimization. In: 2002 14th International Conference on Digital Signal Processing Proceedings. DSP 2002 (Cat. No.02TH8628). [S.I.: s.n.], 2002. v. 2, p. 787-790 vol.2.

Blind image deconvolution via dispersion minimization. Digital Signal Processing, v. 16, p. $137-148,2006$.

WALLACE, W. et al. Deconvolution in optical microscopy. 022018.

WANG, Z. et al. Image quality assessment: from error visibility to structural similarity. v. 13, n. 4, p. 600-612, 2004. ISSN 1057-7149.

XU, L.; JIA, J. Two-phase kernel estimation for robust motion deblurring. In: Proceedings of the 11th European Conference on Computer Vision: Part I. Berlin, Heidelberg: Springer-Verlag, 2010. (ECCV'10), p. 157-170. ISBN 3-642-15548-0, 978-3-642-15548-2. Disponível em: <http://dl.acm.org/citation.cfm?id=1886063.1886077>.

ZHANG, C. et al. Frequency domain decision feedback equalization for uplink sc-fdma. IEEE Transactions on Broadcasting, v. 56, n. 2, p. 253-257, June 2010. ISSN 0018-9316.

ZHAO, H.; ZENG, X.; HE, Z. Low-complexity nonlinear adaptive filter based on a pipelined bilinear recurrent neural network. IEEE Transactions on Neural Networks, v. 22, n. 9, p. 1494-1507, Sept 2011. ISSN 1045-9227.

Low-complexity nonlinear adaptive filter based on a pipelined bilinear recurrent neural network. IEEE Transactions on Neural Networks, v. 22, n. 9, p. 1494-1507, Sept 2011. ISSN 1045-9227. 


\section{APÊNDICE A - O ALGORITMO RMA}

O algoritmo multimódulo regional (regional multimodulus algorithm - RMA) foi proposto em (MENDES-FILHO et al., 2009) com o objetivo de melhorar o desempenho do CMA para equalização de sinais de módulo não constante. O RMA trata os sinais de constelação de módulo não constante como os de constelação de módulo constante, convergindo aproximadamente para a solução de Wiener. Para evitar divergência, as estimativas não consistentes do sinal transmitido são rejeitas. Comparado ao CMA, o RMA exibe um desajuste consideravelmente baixo, convergência mais rápida, e boa capacidade de tracking sem comprometer o custo computacional.

Assumindo a equalização de sinais reais, para o uso posterior em imagens, a atualização dos coeficientes do RMA é dada pela equação

$$
\mathbf{w}(n)=\mathbf{w}(n-1)+\frac{\tilde{\mu}}{\delta+\|\mathbf{u}(n)\|^{2}} \bar{e}(n) \mathbf{u}(n),
$$

em que $\tilde{\mu}$ é o passo de adaptação, $\delta$ é uma constante pequena e positiva para evitar divisão por zero e $\bar{e}(n)$ é o erro de estimação explicado a seguir. Ao considerar o sinal transmitido uma sequência 8-PAM $(N=8)$, em que os símbolos da constelação podem ser representados em uma reta real, definem-se regiões dessa reta contendo dois símbolos, como esquematizado na Figura 62. Os centros das regiões $A_{k}$ para $k=-N / 4, \ldots,-1,1, \cdots, N / 4$ são indicados por $c_{k}$. Para uma constelação $N$-PAM, utiliza-se $\left\lceil\log _{2}(N)-1\right\rceil$ comparações $^{1}$ para identificar a região $A_{k}$ à qual pertence a saída do equalizador $y(n)$. Usando essa informação, o erro do RMA é definido como

$$
\bar{e}(n)=\left|c_{k}\right|\left[1-\bar{y}^{2}(n)\right] \bar{y}(n),
$$

em que $\bar{y}(n)=y(n)-c_{k}$ é a saída $y(n)$ transladada de $c_{k}$, ou simplesmente saída transladada.

\footnotetext{
${ }^{1}\lceil x\rceil$ representa o inteiro mais próximo de $x$.
} 
Figura 62 - Regiões de um sinal 8-PAM.

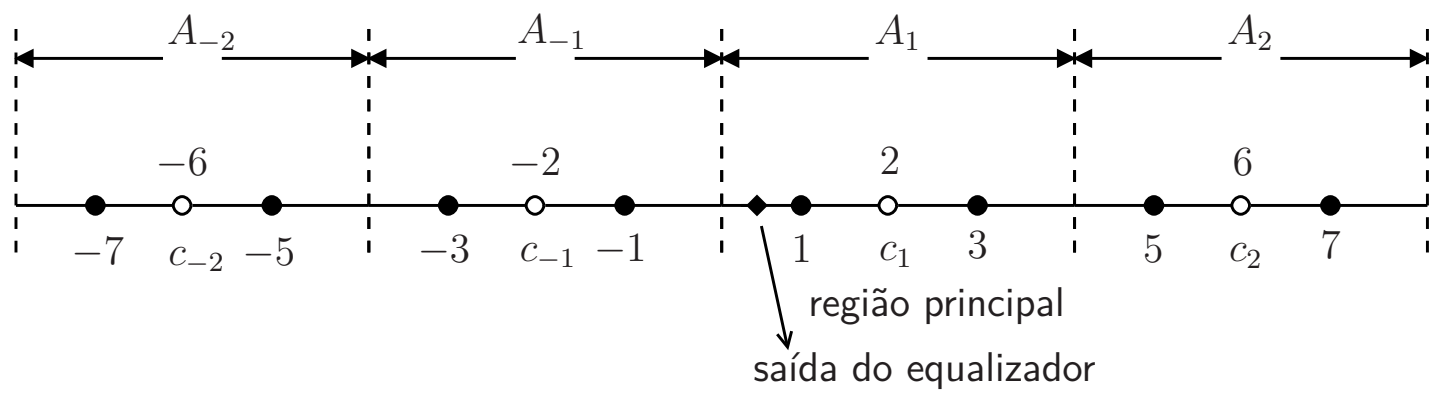

Fonte: Autora.

Ao transladar a saída $y(n)$, o centro da região identificada é deslocado para a origem da reta real, fazendo com que os símbolos dessa região pertençam a uma constelação de módulo constante, assim tudo ocorre como se apenas os símbolos $\{ \pm 1\}$ de uma constelação 2-PAM tivessem sido transmitidos. Por isso, o erro do RMA, na Equação A.2, é semelhante ao erro do CMA com o fator de dispersão $r$ igual a 1. A Figura 63 mostra o erro do CMA e do RMA em função da saída $y(n)$ para um sinal 8-PAM. Diferente do CMA, o erro do RMA é zero quando $y(n)$ é igual a um dos símbolos da constelação, o que faz esse algoritmo funcionar melhor em constelações com mais símbolos. Dessa maneira, o RMA trata constelações de módulo não constante como se fossem de módulo constante, se aproximando da solução de Wiener. As equações que implementam o algoritmo RMA para $N$ iterações estão resumidas na Tabela 11 .

Figura 63 - Erro do CMA (à esquerda) e do RMA (à direita) em função de $y(n)$ para um sinal 8-PAM.
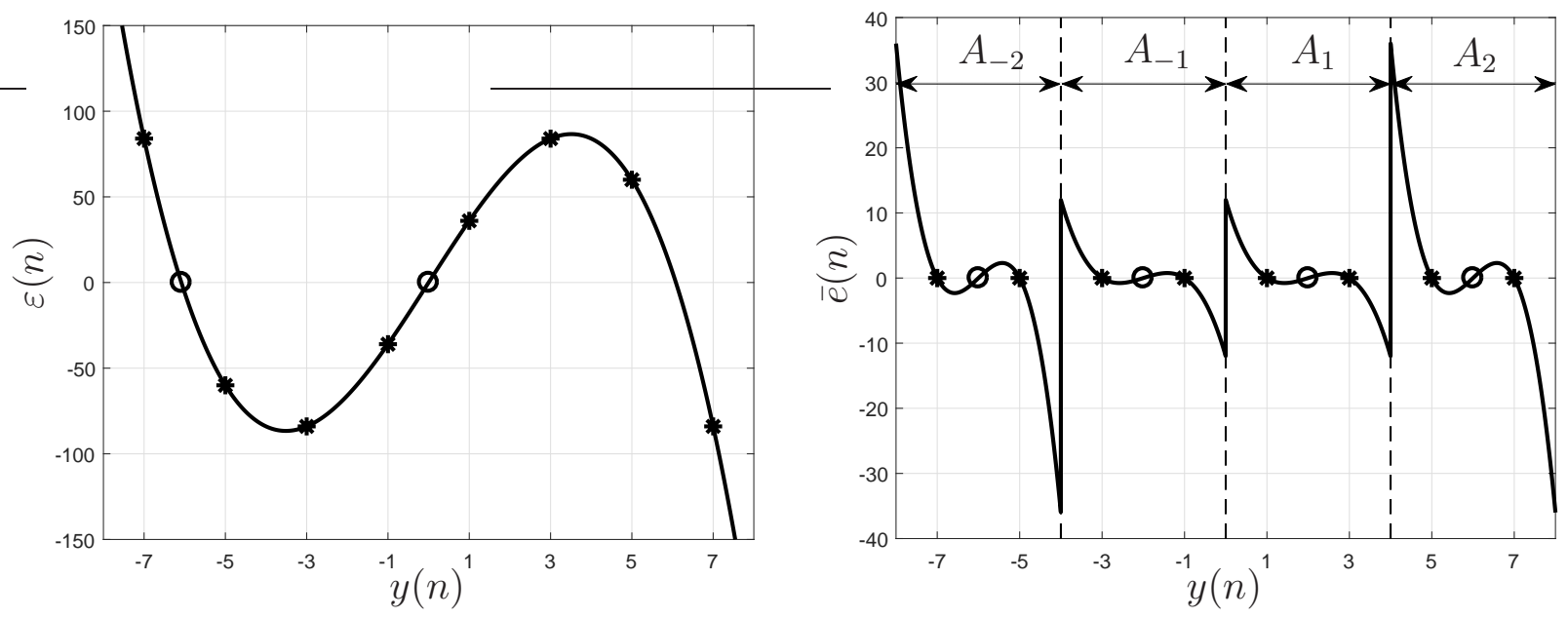

Fonte: Autora. 
Tabela 11: Sumário do algoritmo RMA.

\begin{tabular}{l} 
Algoritmo RMA \\
\hline Escolha do passo de adaptação $\tilde{\mu}>0, \quad r=\frac{\mathrm{E}\left\{a^{4}(n)\right\}}{\mathrm{E}\left\{a^{2}(n)\right\}}$ \\
Inicialização \\
$\mathbf{w}_{1}(0)=\left[\begin{array}{llllllll}0 & \cdots & 0 & 1 & 0 & \cdots & 0\end{array}\right]^{T}$
\end{tabular}

\section{Cálculos}

Para $n=1,2, \cdots, N$, faça:

\% Cálculo da saída:

$y(n)=\mathbf{u}^{T}(n) \mathbf{w}(n-1)$

\% Cálculo do erro:

$\bar{y}(n)=y(n)-c_{k}$
$\bar{x}(n)=1,5-0,5 \bar{y}^{2}(n)$

Se $\bar{x}(n) \geq 0$

$\bar{d}(n)=\bar{x}(n) \bar{y}(n)$

\section{Caso contrário:}

$$
\bar{d}(n)=0
$$

$\bar{e}(n)=\left|c_{k}\right|\left[\bar{d}_{k}(n)-\bar{y}_{k}(n)\right]$

\% Atualização:

$\mathbf{w}(n)=\mathbf{w}(n-1)+\frac{\tilde{\mu}}{\delta+\|\mathbf{u}(n)\|^{2}} \bar{e}(n) \mathbf{u}(n)$

\section{Fim}

Fonte: Autora. 